TALLES BATISTA RATTIS SANTOS

\title{
DESENVOLVIMENTO E APLICAÇÃO DE INFORMAÇÕES ANATÔMICAS E FISIOLÓGICAS A PRIORI PARA OBTENÇÃO DE IMAGENS DE TOMOGRAFIA POR IMPEDÂNCIA ELÉTRICA
}

São Paulo 


\title{
TALLES BATISTA RATTIS SANTOS
}

\section{DESENVOLVIMENTO E APLICAÇÃO DE INFORMAÇÕES ANATÔMICAS E FISIOLÓGICAS A PRIORI PARA OBTENÇÃO DE IMAGENS DE TOMOGRAFIA POR IMPEDÂNCIA ELÉTRICA}

\author{
Versão corrigida
}

Tese apresentada à Escola Politécnica da Universidade de São Paulo para a obtenção do título de Doutor em Ciências

São Paulo 


\section{TALLES BATISTA RATTIS SANTOS}

\section{DESENVOLVIMENTO E APLICAÇÃO DE INFORMAÇÕES ANATÔMICAS E FISIOLÓGICAS A PRIORI PARA OBTENÇÃO DE IMAGENS DE TOMOGRAFIA POR IMPEDÂNCIA ELÉTRICA}

Versão corrigida

Tese apresentada à Escola Politécnica da Universidade de São Paulo para a obtenção do título de Doutor em Ciências

Área de Concentração: Engenharia

Mecânica

Orientador: Prof. Dr. Raul Gonzalez Lima

São Paulo 
Autorizo a reprodução e divulgação total ou parcial deste trabalho, por qualquer meio convencional ou eletrônico, para fins de estudo e pesquisa, desde que citada a fonte.

Este exemplar foi revisado e corrigido em relação à versão original, sob responsabilidade única do autor e com a anuência de seu orientador.

São Paulo, de de

Assinatura do autor:

Assinatura do orientador:

\section{Catalogação-na-publicação}

Santos, Talles Batista Rattis

Desenvolvimento e aplicação de informações anatômicas e fisiológicas a priori para obtenção de imagens de tomografia por impedância elétrica / T. B. R. Santos -- versão corr. -- São Paulo, 2020.

$106 \mathrm{p}$.

Tese (Doutorado) - Escola Politécnica da Universidade de São Paulo. Departamento de Engenharia Mecânica.

1.Tomografia por impedância elétrica 2.Método D-bar 3.Teoria do erro de aproximação 4.Inferência Bayesiana 5.Complemento de Schur I.Universidade de São Paulo. Escola Politécnica. Departamento de Engenharia Mecânica II.t. 


\section{Dedicatória}

Para meus pais João e Míriam, minha irmã Vanessa e minha esposa Glenda. Sem o amor de vocês eu nada seria. 


\section{Agradecimentos}

Inicialmente gostaria de agradecer ao meu orientador Raul Gonzalez Lima, que tanto contribuiu para a minha formação. Guardarei com carinho e apreço todos os seus ensinamentos.

Gostaria também de agradecer à professora Jennifer L. Mueller, a sua contribuição foi imprescindível para que esta tese se tornasse realidade. E também à sua família, que sempre me acolheu com tanto carinho.

Um importante agradecimento à Coordenação de Aperfeiçoamento de Pessoal de Nível Superior (CAPES) e a Escola Politécnica da Universidade de São Paulo (EPUSP) pelo apoio financeiro e suporte dado a este trabalho.

Ao professor Marcelo Amato, agradeço pelo apoio e pela oportunidade de trabalharmos juntos, o seu entusiasmo é contagiante. E ao professor Jari Kaipio, pela luz que as suas ideias trouxeram à este trabalho.

Um agradecimento especial aos meus amigos da Batcaverna Erick León, Fernando Moura, Michelle Mellenthin, Olavo Luppi e Rafael Mikio pelo apoio, amizade e generosidade em dividir conhecimentos e experiências. Expresso aqui o meu carinho e consideração.

Não posso me esquecer dos meus colegas de laboratório Ana Isabel, André, Ely, Luis Henrique e Tayran. Fica aqui o meu muito obrigado.

Por fim, agradeço o carinho incondicional e irrestrito dado pelos meus pais João e Míriam, pela minha irmã Vanessa, pelo meu cunhado e amigo Juá, pela minha amada esposa Glenda e por seus pais Arnaldo e Mariana. 
" Valeu a pena? Tudo vale a pena Se a alma não é pequena.

Quem quer passar além do Bojador Tem que passar além da dor.

Fernando Pessoa 


\section{Resumo}

A tomografia por impedância elétrica (TIE) é um método de imageamento nãoinvasivo com aplicações clínicas variadas, incluindo imageamento da função pulmonar. A técnica de TIE é considerada um problema inverso não linear e mal-posto. A imagem resultante representa a distribuição de impeditividade no domínio e pode ser estimada através de algoritmos de reconstrução iterativos (e.g. Gauss-Newton) e diretos (e.g. D-bar). Alguns algoritmos iterativos de reconstrução de TIE tem sua resolução espacial melhorada utilizando a teoria de problemas inversos estatísticos para introduzir informação anatômica e fisiológica a priori e para compensar erros de modelagem. O método $D$-bar usado para encontrar soluções de problemas inversos pode se beneficiar com a inclusão de priors estatísticos. Neste trabalho, dois métodos diferentes são propostos para introduzir informação estatística a priori no método $D$-bar. O primeiro método incorpora os priors estatísticos no algoritmo $D$-bar através do pré-condicionamento das medidas de tensão utilizando como base a teoria do erro de aproximação e técnicas Bayesianas. Baseado nas propriedades do complemento de Schur, o segundo método propõe o pós-processamento da imagem obtida pelo método $D$-bar pela maximização da função de densidade de probabilidade condicional de uma imagem que é consistente com a informação a priori, dada a imagem que foi estimada através das medidas. As imagens resultantes dos fantomas numéricos e experimentais mostram uma melhora na resolução espacial para ambos os métodos.

Palavras-chave: Tomografia por impedância elétrica, método $D$-bar, teoria do erro de aproximação, inferência Bayesiana, complemento de Schur. 


\begin{abstract}
Electrical impedance tomography (EIT) is a non-invasive imaging technique with a variety of clinical applications, including pulmonary imaging. The EIT technique is considered a non-linear ill-posed inverse problem. The output image represents the impeditivity distribution of the domain and it can be estimated by iterative (e.g. Gauss-Newton) and direct (e.g. D-bar) reconstruction algorithms. Some iterative EIT reconstruction algorithms have their spatial resolution improved by using statistical inverse theory to introduce anatomical and physiological prior information and to compensate for modelling errors. The $D$-bar method used to solve the inverse problem may benefit by the inclusion of statistical priors in the algorithm. In this work, two different methods are proposed to introduce statistical prior information into the $D$-bar method. The first method incorporates the statistical priors in the $D$-bar algorithm by preconditioning the voltage measurements based on the approximation error theory and Bayesian techniques. Based on Schur complement properties, the second method proposes a post-processing of the image obtained by the $D$-bar method by maximizing the conditional probability density function of an image that is consistent with prior information, given a raw image that was computed from measurements. Images resulting from numerical and experimental phantoms show an improved spatial resolution by the use of both methods.
\end{abstract}

keywords: Electrical impedance tomography, D-bar method, approximation error theory, Bayesian inference, Schur complement. 


\section{Lista de Figuras}

2.1 Modelo de eletrodo completo 3D. FONTE: figuras extraídas de Moura $[17$, Seç. A.1.3 $\ldots$. . . . . . . . . . . . . . . . . . . . 30

3.1 Diagrama que descreve o método de introdução de informação a priori através do pós-processamento das imagens reconstruídas. . . . . . . 48

4.1 Malha problema direto simplificado, $13 \mathrm{k}$ tetraedros. . . . . . . . . . . 54

4.2 Tensão nos eletrodos, Padrão 1 . . . . . . . . . . . . . . . . . . 54

4.3 Estimativa inicial, modelo simplificado. . . . . . . . . . . . . . 55

4.4 Malha problema direto refinado, $126 k$ tetraedros . . . . . . . . . 56

4.5 Cálculo da tensão nos eletrodos utilizando a malha refinada. . . . . . . 56

4.6 Cálculo do erro de aproximação $\tilde{e}_{0}$, pula-0 e Padrão $1 \ldots \ldots$. . . . 57

4.7 Estimativa inicial com o erro de aproximação, malha simplificada. . . . . 57

4.8 Fantoma experimental, cuba circular com 3 pepinos. . . . . . . . . . . . 59

4.9 Amostras simuladas, fatias de pepino. . . . . . . . . . . . . . 59

4.10 Caso 1, fantoma com pepinos posicionados em linha. . . . . . . . . . 61

4.11 Caso 1, fantoma com pepinos posicionados em forma de triângulo. . . . 61

4.12 Caso 2, fantoma com pepinos posicionados em linha. . . . . . . . . 62

4.13 Caso 2, fantoma com pepinos posicionados em forma de triângulo. . . . 63

4.14 Fantoma experimental, cuba em formato de tórax humano com órgãos feitos de ágar. . . . . . . . . . . . . . . . . . 64

4.15 Identificação dos contornos dos órgãos e da cuba. . . . . . . . . . . 64

4.16 Malha de elementos finitos em formato de tórax humano, $13 \mathrm{k}$ elementos 65

4.17 Processo de identificação das regiões na malha de elementos finitos. . . . 65

4.18 Simulação das amostras do prior. . . . . . . . . . . . . . . 66 
4.19 Caso 1, fantoma com cuba em formato de tórax humano com órgãos de ágar intactos. . . . . . . . . . . . . . . . . . . 68

4.20 Caso 1, fantoma com cuba em formato de tórax humano com pulmão de ágar direito cortado.

4.21 Caso 2, fantoma com cuba em formato de tórax humano com órgãos de ágar intactos. . . . . . . . . . . . . . . . . . . 70

4.22 Caso 2, fantoma com cuba em formato de tórax humano com pulmão de ágar direito cortado. . . . . . . . . . . . . . . . . . . . 71

4.23 Caso 1, comparação dos resultados para cuba em formato de tórax humano com órgãos de ágar. . . . . . . . . . . . . . . . . . . . . 72

4.24 Caso 2, comparação dos resultados para cuba em formato de tórax humano com órgãos de ágar. . . . . . . . . . . . . . . . . . . . 73

4.25 Caso 1, remoção do pulmão direito. . . . . . . . . . . . . . . . . . . 76

4.26 Caso 2 , rotação dos pulmões para fora. . . . . . . . . . . . . . . 76

4.27 Caso 3, rotação dos pulmões para dentro. . . . . . . . . . . . . . 77

4.28 Caso 4, rotação pulmão direito para dentro e pulmão esquerdo para fora. 77

4.29 Caso 5, pequeno corte na base do pulmão direito. . . . . . . . . . . . 78

4.30 Caso 6, corte médio na base do pulmão direito. . . . . . . . . . . . 78

4.31 Caso 7, corte grande na base do pulmão direito. . . . . . . . . . . . . . 79

4.32 Caso 8, pequeno corte topo do pulmão direito. . . . . . . . . . . . . . 79

4.33 Caso 9 , corte grande no topo do pulmão direito. . . . . . . . . . . . 80

4.34 Caso 10, redução tamanho do pulmão esquerdo. . . . . . . . . . . . . . . 80

4.35 Fantoma experimental, cuba em formato de tórax humano com coração de ágar e pulmões de melancia. . . . . . . . . . . . . . . . . . . . 82

4.36 Imagens absolutas, cuba em formato de tórax humano com coração de ágar e pulmão de melancia. . . . . . . . . . . . . . . . . . . . 83

4.37 Imagens absolutas, cuba em formato de tórax humano com coração de ágar e pulmão de melancia com o pós-processamento das imagens. . . .

4.38 Diferença entre imagens absolutas para corte pequeno no pulmão direito, cuba em formato de tórax humano com coração de ágar e pulmão de melancia com o pós-processamento das imagens. . . . . . . . . . . . 
4.39 Diferença entre imagens absolutas para corte grande no pulmão direito, cuba em formato de tórax humano com coração de ágar e pulmão de melancia com pós-processamento das imagens. . . . . . . . . . . . . 86

4.40 Imagens relativas, cuba em formato de tórax humano com coração de ágar e pulmão de melancia. . . . . . . . . . . . . . . . . . . . 87

4.41 Imagens relativas, distribuição de cond. estimada, cuba em formato de tórax humano com coração de ágar e pulmão de melancia com o pósprocessamento das imagens. . . . . . . . . . . . . . . . . . 88

4.42 Diferença entre imagens relativas para corte pequeno no pulmão direito, cuba em formato de tórax humano com coração de ágar e pulmão de melancia com o pós-processamento das imagens. . . . . . . . . . . 8

4.43 Diferença entre imagens relativas para corte grande no pulmão direito, cuba em formato de tórax humano com coração de ágar e pulmão de melancia com o pós-processamento das imagens. . . . . . . . . . . . . . 90 


\section{Lista de símbolos}

$\Gamma_{s w} \quad$ Matriz de covariância estimada de um tórax suíno

$\pi(\sigma) \quad$ Função densidade de probabilidade a priori de $\sigma$

$\pi\left(\vec{\phi}_{m} \mid \sigma\right)$ Função verossimilhança

$\pi\left(\vec{\phi}_{m}\right)$ Função verossimilhança marginal

$\pi\left(\sigma \mid \vec{\phi}_{m}\right)$ Função densidade de probabilidade de $\sigma$ dado o evento observado $\vec{\phi}_{m}$

a Vetor arbitrário de tamanho $n_{a}$

$\rho_{0_{s w}}$ Distribuição média de resistividade estimada de um tórax suíno.

A Matriz positiva e semidefinida arbitrária

C Valor da capacitância do eletrodo de medição

E Variável aleatória Gaussiana que descreve o erro de medição $e$

F $\quad$ Filtro gaussiano espacial passa-altas

I Matriz identidade

$I_{e} \quad$ Corrente total que atravessa o eletrodo

$J_{i} \quad$ Matriz Jacobina de $\vec{\phi}_{c}\left(\vec{\sigma}_{i}\right)$

L Matriz de regularização de de Tikhonov generalizada

$N_{\text {tot }} \quad$ Número de amostras utilizadas

$P \quad$ Operador linear que leva o problema direto para o problema direto simplificado

$S_{e} \quad$ Superfície do eletrodo 
$V \quad$ Variável aleatória Gaussiana que descreve a distribuição de tensão $v$

X Variável aleatória Gaussiana

Y Variável aleatória Gaussiana

$Y(\vec{\sigma})$ Matriz de condutividade global utilizando o MEF

$Y_{\text {local }}^{\text {cap }}$ Matriz local de um elemento concentrado capacitivo

$Z_{c} \quad$ Impedância de um capacitor

$\Gamma \quad$ Matriz simétrica positiva definida dividida em blocos

$\Gamma_{11} \quad$ Matriz arbitrária que representa o bloco superior esquerdo da matriz $\Gamma$

$\Gamma_{12}$ Matriz arbitrária que representa o bloco superior direito da matriz $\Gamma$

$\Gamma_{21} \quad$ Matriz arbitrária que representa o bloco inferior esquerdo da matriz $\Gamma$

$\Gamma_{22}$ Matriz arbitrária que representa o bloco superior direito da matriz $\Gamma$

$\Gamma_{\text {noise }}$ Matriz de covariância do erro de medição $e$

$\Gamma_{p r} \quad$ Matriz de covariância da distribuição de condutividade $\sigma$

$\Gamma_{x x} \quad$ Matriz de covariância da variável $x$

$\Gamma_{x y} \quad$ Matriz de covariância cruzada entre as variáveis $x$ e $y$

$\Gamma_{y x} \quad$ Matriz de covariância cruzada entre as variáveis $y$ e $x$

$\Gamma_{y y} \quad$ Matriz de covariância da variável $y$

$\Gamma_{z} \quad$ Matriz de covariância da distribuição $z$

$\Omega \quad$ Domínio de forma geométrica conhecida

$\Sigma \quad$ Variável aleatória Gaussiana que descreve a distribuição de condutividade $\sigma$

$\Sigma_{\text {MAP }}$ Distribuição estimada de $\Sigma$ através do MAP

$\alpha_{i} \quad$ Tamanho do passo na iteração $i$

$\beta \quad$ Parâmetro de regularização associado ao atlas anatômico na tensão 
$\gamma \quad$ Parâmetro de regularização associado ao atlas anatômico

$\kappa \quad$ Parâmetro de controle do nível de ruído branco adicionado

$\lambda \quad$ Parâmetro de regularização de Tikhonov

$\mu_{x} \quad$ Expectância da variável $x$

$\mu_{y} \quad$ Expectância da variável $y$

$\mu_{z} \quad$ Expectância da variável $z$

$\partial \Omega \quad$ Superfície que delimita o contorno do domínio $\Omega$

$\phi(\vec{r}, t)$ Distribuição de potencial elétrico

$\pi(v) \quad$ Função verossimilhança marginal

$\pi\left(v_{c}\right)$ Função densidade de probabilidade $a$ priori de $v_{c}$

$\sigma \quad$ Distribuição de condutividade no meio, para o modelo $f($.

$\sigma^{3 D} \quad$ Conjunto de amostras de distribuições de condutividades tridimensionais

$\sigma_{0} \quad$ Expectância da distribuição de condutividade $\sigma$

$\chi \quad$ Reatividade elétrica (parte imaginária de $\zeta$ )

$\rho \quad$ Resistividade elétrica (parte real de $\zeta$ )

$\boldsymbol{\sigma} \quad$ Condutividade elétrica

$\zeta \quad$ Impeditividade elétrica

n $\quad$ Versor normal apontando para fora do meio $\Omega$

$\tilde{E} \quad$ Variável aleatória Gaussiana que descreve o erro $\tilde{e}$

$\tilde{\Gamma}_{n o i s e}$ Matriz de covariância do erro $\tilde{e}$

$\tilde{\Gamma}_{p r_{v}} \quad$ Matriz de covariância do conjunto de tensões $v_{c}$

$\tilde{\Gamma}_{p r} \quad$ Matriz de covariância da distribuição de condutividade $\tilde{\sigma}$

$\tilde{\Sigma} \quad$ Variável aleatória Gaussiana que descreve a distribuição de condutividade $\tilde{\sigma}$ 
$\tilde{\sigma} \quad$ Distribuição de condutividade no meio, para o modelo simplificado $\tilde{f}($.)

$\tilde{\sigma}_{0} \quad$ Expectância da distribuição de condutividade $\tilde{\sigma}$

$\tilde{e}_{0} \quad$ Expectância do erro de $\tilde{e}$

$\tilde{f}($.$) \quad Modelo determinístico simplificado do problema direto$

$\tilde{v_{0}} \quad$ Média do conjunto de tensões $v_{c}$

$\vec{\sigma} \quad$ Vetor de condutividades dos elementos da malha

$\vec{C}_{p} \quad$ Vetor de correntes nodais que excitam o domínio segundo o padrão de excitação $p$

$\vec{\phi}_{c}(\vec{\sigma})$ Vetor de tensões calculadas nos $n_{e}$ eletrodos para os $n_{p}$ padrões de excitação

$\vec{\phi}_{m} \quad$ Conjunto de tensões medidas utilizada para estimar uma distribuição de impeditividade (imagem)

$\vec{\rho}^{*} \quad$ Vetor com a distribuição de resistividade esperada na malha de elementos finitos

$\vec{\sigma}^{*} \quad$ Vetor com a distribuição de condutividade esperada na malha de elementos finitos

$\vec{r} \quad$ Vetor posição em um sistema de coordenadas cartesianas

$\zeta \quad$ Distribuição de impeditividade elétrica no material

e $\quad$ Erro de medição

$e_{0} \quad$ Expectância do erro de medição $e$

f $\quad$ Frequência do sinal de excitação

f(.) Modelo determinístico que descreve o problema direto

$i \quad$ Iteração atual do método de Gauss-Newton

j Unidade imaginária

$m \quad$ Número de elementos da malha de elementos finitos

$n_{e} \quad$ Número de eletrodos 
$n_{p} \quad$ Número de padrões de excitação

$n_{a} \quad$ Número inteiro positivo relacionada ao tamanho do vetor $a$ e da matriz $A$

$n_{\text {nos }} \quad$ Número de nós da malha de elementos finitos

$n_{\text {ref }}$ Dimensão da malha de elementos finitos do problema direto

$n_{\text {sim }}$ Dimensão da malha de elementos finitos do problema direto simplificado

$p \quad$ Padrão de excitação utilizado

$v \quad$ Distribuição de tensão em um domínio

$v_{c} \quad$ Medidas pré-condicionadas

$x \quad$ Evento associado a variável aleatória $X$

$x_{i} \quad$ Imagem $\sigma^{2 D}$ vetorizada

$x_{v} \quad$ Vetor de tamanho $n$

y Evento associado a variável aleatória $Y$

$y_{i} \quad$ Imagem $\tilde{\sigma}_{D b a r}^{2 D}$ vetorizada

$y_{v} \quad$ Vetor de tamanho $n$

$z \quad$ Conjunto de amostras $z_{1: N_{t o t}}$

$z_{i} \quad$ Vetor que representa os vetores $x_{i}$ e $y_{i}$ concatenados

$Z_{C_{\text {media }}}$ Efeito capacitivo médio do eletrodo

$\Gamma_{\sigma}^{3 D}$ Matriz de covariância da distribuição de condutividade tridimensional

$\bar{\sigma}^{3 D}$ Expectância da distribuição de condutividade tridimensional

$\sigma^{2 D}{ }_{i}$ Distribuição de condutividade na seção transversal onde os eletrodos estão posicionados da $i$-ésima amostra de distribuição de condutividade tridimensional

$\sigma^{3 D}{ }_{i} \quad i$-ésima amostra de distribuição de condutividade tridimensional

$\sigma_{i m p \_c o n t}$ Impedância de contato média do modelo de eletrodo 
$\sigma_{\text {meio }}$ Condutividade média do meio (domínio)

$\tilde{\Gamma}_{11} \quad$ Complemento de Schur de $\Gamma_{11}$

$\tilde{\Gamma}_{22} \quad$ Complemento de Schur de $\Gamma_{22}$

$\vec{V}_{p}^{\prime} \quad$ Tensões calculadas nos nós que representam os eletrodos através do MEF

$\vec{V}_{p} \quad$ Vetor de potenciais nos nós da malha de elementos finitos para o padrão $p$

$\tilde{\sigma}_{\text {Dbar } i}^{2 D}$ Imagem $\sigma^{2 D}{ }_{i}$ quando reconstruída através do método $D$-bar 


\section{Conteúdo}

$\begin{array}{ll}\text { Lista de Figuras } & 9\end{array}$

1 Introdução $\quad 20$

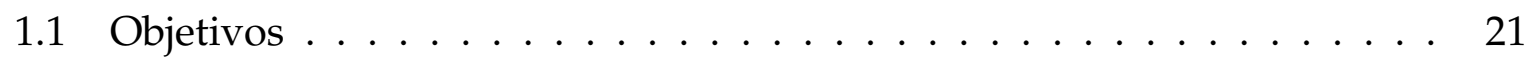

2 Tomografia por Impedância Elétrica 22

2.1 Tomógrafo ACE1 . . . . . . . . . . . . . . . . . . 23

2.2 Modelagem ............................ 24

2.2.1 Equacionamento do problema eletromagnético . . . . . . . . . . 24

2.2.2 Solução do problema direto . . . . . . . . . . . . . . 25

2.2.3 Teoria do erro de aproximação . . . . . . . . . . . . . 26

2.2.4 Modelos de eletrodo . . . . . . . . . . . . . . . . . 29

2.2.5 Modelagem do efeito capacitivo nos eletrodos . . . . . . . . . . 30

2.3 Solução do problema inverso . . . . . . . . . . . . . . . . 32

2.3.1 Método de Gauss-Newton . . . . . . . . . . . . . . . . . 33

2.3.2 Método D-bar ......................... 34

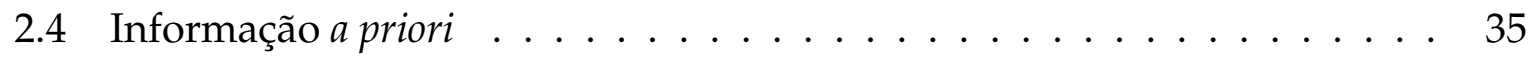

2.4 .1 Atlas anatômico de tórax suíno . . . . . . . . . . . . 37

2.4.2 Informação a priori no método $D$-bar . . . . . . . . . . . . . . 38

2.5 Complemento de Schur . . . . . . . . . . . . . . . . . . 39

2.5.1 Propriedade do complemento de Schur para distribuições Gaussianas......................... 40

2.5.2 Estimativa do máximo a posteriori . . . . . . . . . . . . 40

3 Método $\quad 42$

3.1 Introdução de informação a priori através do ajuste da tensão . . . . . . . 43 
3.1.1 Formulação probabilística para estimação das medidas . . . . . . 44

3.2 Introdução de informação a priori através do pós-processamento das imagens reconstruídas . . . . . . . . . . . . . . . . . 46

3.3 Ensaios para avaliação dos métodos . . . . . . . . . . . . . . . . 49

3.3.1 Ensaios em cuba homogênea . . . . . . . . . . . . . . . 49

3.3.2 Prior baseado em amostras simuladas . . . . . . . . . . . 51

3.3.3 Fantoma numérico ....................... 51

3.3.4 Fantoma experimental ................... 51

4 Resultados e Discussões

4.1 Modelagem do efeito capacitivo . . . . . . . . . . . . . . 53

4.2 Estimativa inicial e cálculo do erro de aproximação . . . . . . . . . . 54

4.3 Introdução de informação a priori através do ajuste da tensão . . . . . . . 58

4.3.1 Fantoma experimental - Cuba circular e pepinos . . . . . . . 5 58

4.3.2 Fantoma experimental - Cuba em formato de tórax humano e objetos feitos de ágar . . . . . . . . . . . . . . . 63

4.4 Introdução de informação a priori através do pós-processamento das imagens reconstruídas . . . . . . . . . . . . . . . 71

4.4.1 Prior baseado em amostras simuladas . . . . . . . . . . . 72

4.4.2 Cálculo dos parâmetros A e b . . . . . . . . . . . . 74

4.4 .3 Fantoma numérico . . . . . . . . . . . . . . . 75

4.4.4 Fantoma experimental . . . . . . . . . . . . 81

5 Conclusões $\quad 92$

A Complemento de Schur $\quad 94$

A.1 Equacionamento do complemento de Schur . . . . . . . . . . . . . . . . . 94

A.1.1 Definição . . . . . . . . . . . . . . . . . . . . 94

A.1.2 Lema . . . . . . . . . . . . . . . . . 95

A.2 Complemento de Schur e densidade de probabilidade condicional . . 97

A.2.1 Demonstração . . . . . . . . . . . . . . . . . . . . . . . 97

A.3 Estimativa do máximo a posteriori . . . . . . . . . . . . . 100

$\begin{array}{ll}\text { Bibliografia } & 102\end{array}$ 


\section{Capítulo 1}

\section{Introdução}

A Tomografia por impedância elétrica (TIE) é um método não invasivo para obtenção de imagens que baseia-se na estimação da distribuição de impeditividade elétrica ${ }^{1}$ de um domínio a partir da imposição de correntes elétricas (ou potenciais) e medição de potenciais (ou correntes) ao longo de sua fronteira. Esta técnica é considerada um problema inverso [1, 2], não linear e mal-posto. Diferentes métodos para a estimação da distribuição de impeditividades foram propostos [3], sendo divididos entre iterativos, como o Gauss-Newton [4], e diretos, como o D-bar [5].

Para a solução do problema de TIE geralmente são utilizados métodos iterativos que linearizam e regularizam o problema. O método de Gauss-Newton é comumente utilizado para a solução de problemas de mínimos quadrados não lineares. Este método aproxima a função objetivo do problema por meio de um sistema linearizado e, para que este sistema admita solução única e estável, utiliza a regularização de Tikhonov generalizada [4]. Sob a ótica Bayesiana a regularização pode ser vista como uma informação a priori e a função objetivo pode ser descrita como a função densidade de probabilidade a posteriori.

Por enquanto, poucos são os métodos diretos para a solução de problemas inversos não lineares. Em seu livro, Mueller e Siltanen [6] destacam como as principais vantagens dessa família de algoritmos a capacidade de encontrar a solução do problema de forma direta (não iterativa) e sem a possibilidade do algoritmo encontrar um mínimo local, a viabilidade da reconstrução de regiões de interesse específicas e a fácil

\footnotetext{
${ }^{1} \mathrm{~A}$ impeditividade é uma propriedade elétrica dos materiais e pode ser descrita no plano complexo por $\zeta=\rho+j \chi$, onde a parte real $\rho$ é chamada resistividade e a parte imaginaria $\chi$ é chamada reatividade.
} 
implementação da paralelização nestes algoritmos. Atualmente, segundo estes mesmos autores, o método direto mais desenvolvido para a solução do problema de TIE é o método D-bar.

Segundo Kaipio e Somersalo [7], um dos passos mais cruciais na teoria Bayesiana dos problemas inversos é a construção de priors para regularização do sistema. As considerações acerca do modelo determinístico e as regularizações adotadas para a construção do modelo estatístico são fundamentais para o bom desempenho do método computacional utilizado. Em sua tese de doutorado, na qual foi desenvolvido e validado um atlas anatômico de tórax suíno para TIE, Camargo [8] mostrou que a inclusão de priors estatísticos melhora significativamente a qualidade de imagens absolutas do tórax suíno.

Em Camargo [8] foi demostrado que a inclusão de um atlas anatômico, ou informação a priori baseada em amostras, para a regularização do problema inverso em métodos iterativos, por exemplo Gauss-Newton, mostrou-se eficiente para a obtenção e melhoria de imagens absolutas. No entanto a utilização de priors estatísticos em métodos diretos, como o D-bar, ainda são pouco explorados. Até o presente momento, no conhecimento do autor desta tese, não existem trabalhos publicados para a utilização de priors estatísticos sob a ótica Bayesiana na estimação da distribuição de impeditividade em um domínio utilizando o método $D$-bar.

\subsection{Objetivos}

O objetivo deste trabalho é o desenvolvimento e aplicação de priors estatísticos, segundo a estatística Bayesiana, para obtenção de melhores imagens de tórax utilizando o método D-bar.

Sob a ótica Bayesiana, propõe-se implementar e avaliar, para o método $D$-bar, formas de considerar a densidade de probabilidade da distribuição da condutividade de uma população para gerar imagens com melhor resolução espacial. 


\section{Capítulo 2}

\section{Tomografia por Impedância Elétrica}

A Tomografia por impedância elétrica é uma modalidade de imageamento que procura estimar a distribuição de impeditividade no interior de uma região a partir da excitação e medição de correntes e potenciais elétricos na fronteira do domínio. Esta técnica de obtenção de imagens é usada quando existe uma relação entre a propriedade física de interesse e a distribuição de impeditividade da região analisada. Existem várias aplicações para a TIE $^{2}$, clínicas e não clínicas, destacando-se o monitoramento das funções pulmonares [11], para aplicações médicas, e testes não destrutivos em estruturas de concreto [12] e monitoramento de escoamento multifásicos [13], para aplicações industriais.

Atualmente as imagens clínicas para monitoramento do sistema cardiopulmonar realizadas por tomografia computadorizada por raio-x ou ressonância magnética possuem resolução espacial melhor que as de TIE. No entanto, esses métodos apresentam baixa resolução temporal, sendo pouco informativos quanto à dinâmica deste sistema e pouco sensíveis à função dos órgãos envolvidos, por exemplo, no tecido pulmonar, estas técnicas são pouco sensíveis quanto ao conteúdo e variação de gás e sangue nos pulmões.

Quando comparada à outros métodos de imageamento a TIE destaca-se por ser de baixo-custo, não invasiva, não destrutiva, portátil, por ter alta resolução temporal e por não utilizar radiação ionizante. A principal desvantagem desta técnica está relacionada à resolução espacial das imagens estimadas.

\footnotetext{
${ }^{2}$ Uma lista das principais aplicações desta modalidade pode ser encontrada em Holder [9, Part 3 ] e Brown [10].
} 


\subsection{Tomógrafo ACE1}

O Active Complex Electrode EIT System (ACE1) ${ }^{3}$ é um tomógrafo de TIE composto de 32 eletrodos por onde pode-se injetar correntes elétricas de baixa intensidade e frequência variável e medir os potenciais elétricos resultantes desta excitação. Este tomógrafo é capaz de gerar até 33.2 frames por segundo quando utiliza 32 eletrodos para medição das tensões e correntes elétricas envolvidas.

O sistema de excitação deste tomógrafo é composto por um gerador de função e uma fonte de corrente Howland bipolar (ou monopolar [15]) que se conecta à qualquer par de eletrodos através de um sistema de multiplexação. O sistema de medição utiliza 2 placas de aquisição ICS-1640, compondo ao todo 32 conversores analógico-digital com taxa de amostragem de $2.5 \mathrm{MHz}$ e 24 bits de precisão.

As 2 placas de aquisição ICS-1640 trabalham em sincronia para medir ao mesmo tempo os 32 eletrodos e assim serem capazes de obter os 32 valores de amplitude e fase simultaneamente. Para a medição das correntes de excitação do domínio é utilizado no ACE1 o Active Complex Electrode, esse sistema é capaz de medir amplitude e fase das correntes que são injetadas e retiradas do domínio com o mesmo referencial de fase das medidas de tensão dos eletrodos. Uma das principais características deste tomógrafo é que correntes e tensões tenham o mesmo referencial de fase para uma correta estimação das partes real e imaginária das imagens de impeditividade.

O Active Complex Electrode utiliza um sistema composto por chaves digitais e resistores sentinelas. Tais resistores são colocados em série entre a fonte de corrente e os eletrodos e, medindo-se a queda de tensão nestes resistores sentinelas, é possível calcular a corrente que é injetada através de todos os eletrodos. Este conjunto de chaves digitais introduz uma capacitância para o terra em cada eletrodo de aproximadamente 56 pF [15]. Esta capacitância parasita deve ser levada em consideração quando a distribuição de impeditividade é estimada, uma vez que introduz um viés importante nas tensões medidas.

\footnotetext{
${ }^{3}$ Este tomógrafo foi desenvolvido em uma parceria do Laboratório de Engenharia Ambiental e Biomédica, Departamento de Engenharia Mecânica, EPUSP com o EIT lab, Department of Mathematics at Colorado State University. A descrição completa do tomógrafo e testes de desempenho são encontrados em Mellenthin et al. [14].
} 
Para obter um conjunto de medidas $\vec{\phi}_{m}$, um padrão de corrente ${ }^{4}$ é especificado e 32 valores complexos de potencial elétrico e dois valores complexos de corrente são realizados. Em cada conjunto de excitação/medição, o sistema de excitação impõe a corrente em um par de eletrodos e então é medido, através de eletrodos ativos e sistema de medição, o módulo e fase das tensões nos 32 eletrodos e o módulo e fase das correntes nos 2 eletrodos utilizados para a excitação do domínio. A distribuição de impeditividades é estimada a partir de um frame que agrega as medidas resultantes de 32 padrões de excitação.

\subsection{Modelagem}

\subsubsection{Equacionamento do problema eletromagnético}

As equações de Maxwell descrevem fenômenos eletromagnéticos e são a base da teoria do eletromagnetismo clássico. A partir destas equações e utilizando simplificações acerca das propriedades elétricas do material e do domínio é possível mostrar que a distribuição de potencial elétrico $\phi(\vec{r}, t)$ no interior de um domínio de forma geométrica conhecida $\Omega$ pode ser descrito pela equação generalizada de Laplace,

$$
\nabla \cdot\left(\frac{1}{\zeta} \nabla \phi(\vec{r}, t)\right)=0
$$

onde $\zeta$ é a distribuição de impeditividade do meio e $\vec{r}$ o vetor posição em um sistema de coordenadas cartesianas. Com o uso deste modelo admite-se que as propriedades elétricas são isotrópicas, lineares e não dispersivas e que os efeitos de indução magnética podem ser desprezados, na frequência de excitação deste trabalho, $125 \mathrm{kHz}$.

Para a solução da equação generalizada de Laplace é necessário descrever as equações de contorno na fronteira $\partial \Omega$. Assumindo que a excitação do domínio é realizada apenas através dos eletrodos dispostos na fronteira e que não há fontes ou sorvedores de corrente elétrica dentro do volume pode-se utilizar o modelo Gap model descrito

\footnotetext{
${ }^{4} \mathrm{O}$ termo padrão de corrente é utilizado para indicar como as correntes elétricas são impostas para excitação do domínio. Em Malmivuo e Plonsey [16, Seç. 26.2] e Mueller e Siltanen [6, Seç. 12.7] são apresentados diferentes tipos de padrões de corrente e medição.
} 
pela equação

$$
\text { Gap model } \begin{cases}\int_{S_{e}} \zeta^{-1} \frac{\partial \phi}{\partial \hat{\mathbf{n}}} d S=I_{e}, & \text { superfície do eletrodo } \\ \zeta^{-1} \frac{\partial \phi}{\partial \hat{\mathbf{n}}}=0, & \text { demais pontos da fronteira }\end{cases}
$$

onde $I_{e}$ é a corrente total que atravessa o eletrodo, $S_{e}$ é a superfície do eletrodo e $\hat{\mathbf{n}}$ é um versor normal apontando para fora do meio $\Omega$.

Neste trabalho apenas a parte real da distribuição de impeditividade no meio será contemplada, doravante todos os equacionamentos são descritos considerando apenas a distribuição de resistividade (ou condutividade ${ }^{5}$ ) em $\Omega$. A formulação completa do problema eletromagnético e todas as simplificações utilizadas podem ser encontradas em Vauhkonen [4, Cap 3], Moura [17, Cap 2] e Camargo [8, Ap. A].

\subsubsection{Solução do problema direto}

A partir da equação 2.1, utilizando as condições de contorno da equação 2.2 e com o conhecimento da distribuição de condutividade $\sigma$ é possível calcular analiticamente a distribuição de potencial elétrico $\phi(\vec{r})$ no domínio $\Omega$. No entanto, a solução analítica desta equação diferencial parcial é conhecida apenas para algumas distribuições de condutividade $\sigma$ e contornos $\Omega$ específicos. Para obter soluções a partir de distribuições de condutividade arbitrárias é necessário o uso de métodos numéricos.

Para a solução da equação 2.1 com distribuições de condutividade arbitrárias é comumente utilizado na TIE o método dos elementos finitos (MEF). Com este método o domínio $\Omega$ pode ser discretizado em uma malha de elementos finitos e a distribuição do potencial elétrico em cada um dos nós desta malha pode ser calculada quando conhecida a condutividade de cada elemento e as correntes injetadas no domínio.

A equação 2.3 mostra como pode ser calculado o problema direto, onde $\vec{V}_{p} \in \mathbb{C}^{n_{\text {nos }}}$ é o vetor de potenciais nos nós da malha de elementos finitos para o padrão $p$ de excitação, $[Y(\vec{\sigma})] \in \mathbb{C}^{n_{\text {nos }} \times n_{\text {nos }}}$ a matriz de condutividade global, $n_{\text {nos }}$ o número de nós da malha, $\vec{\sigma} \in \mathbb{R}^{m}$ o vetor de condutividades dos elementos da malha, $m$ o número de elementos da malha e $\vec{C}_{p} \in \mathbb{C}^{n_{\text {nos }}}$ o vetor de correntes nodais que excitam o domínio segundo o padrão de excitação $p$.

\footnotetext{
${ }^{5}$ A condutividade $\sigma$ é relacionada com a resistividade por $\sigma=\frac{1}{\rho}$.
} 


$$
\vec{V}_{p}=[Y(\vec{\sigma})]^{-1} \cdot \vec{C}_{p}
$$

Neste trabalho, o domínio é discretizado por malhas 3D formadas por elementos tetraédricos. A demonstração da solução da equação generalizada de Laplace para o problema de TIE através do MEF pode ser encontrada em detalhes em Vauhkonen [4, Cap. 3], Moura [17, Ap. A] e Camargo [8, Ap. B].

Para estimar a distribuição de condutividade do domínio, abordado em detalhes na Seção 2.3, é necessário calcular as tensões nos eletrodos para os diferentes padrões de excitação $p$ aplicados e medidos pelo tomógrafo. Para cada par de correntes de excitação obtém-se, através da solução do problema direto, o valor das tensões $\vec{V}_{p}$ em todos os nós da malha.

A partir do vetor $V_{p}$ são selecionadas as tensões calculadas nos nós que representam os eletrodos $\vec{V}_{p}^{\prime} \in \mathbb{C}^{n e}$ e então é construído o vetor de tensões calculadas $\vec{\phi}_{c}(\vec{\sigma})$,

$$
\vec{\phi}_{c}(\vec{\sigma})=\left[\begin{array}{c}
\vec{V}_{1}^{\prime} \\
\vec{V}_{2}^{\prime} \\
\cdots \\
\vec{V}_{32}^{\prime}
\end{array}\right]_{\left(n_{e} . n_{p}\right) \times 1},
$$

onde $n_{e}$ é o número de eletrodos e $n_{p}$ o número de padrões de excitação.

\subsubsection{Teoria do erro de aproximação}

Segundo Kaipio e Somersalo [7, Seç 5.8] utilizar um modelo reduzido para representar um processo estocástico pode ser considerado um crime inverso. Na TIE modelos que apresentam uma boa acurácia têm elevado custo computacional sendo necessário muitas vezes a utilização de modelos simplificados. No entanto, a utilização de modelos simplificados pode introduzir erros significativos que agem no sistema prejudicando a estimação das distribuições de condutividade.

Uma forma de corrigir modelos simplificados é levar em consideração a estatística da discrepância da soluções do modelo acurado e do modelo reduzido. Kaipio e Somersalo [7, p. 181] propõem calcular as estatísticas deste erro de aproximação e utilizá-las para a solução do problema de estimação. 
Assumindo que a distribuição da tensão no domínio é representada por um modelo que de fato descreve o fenômeno físico, o problema direto por ser descrito por

$$
v=f(\sigma)+e
$$

onde $v$ é a distribuição de tensão, $f($.$) o modelo determinístico do problema direto, e$ o erro de medição e $\sigma$ a distribuição de condutividade no domínio.

Assumindo que a distribuição de condutividades $\sigma$ e o erro de medição $e$ são variáveis aleatórias Gaussianas com estatísticas conhecidas temos

$$
\begin{gathered}
V=f(\Sigma)+E \\
\sigma \sim N\left(\sigma_{0}, \Gamma_{p r}\right) \quad \text { e } \quad e \sim N\left(e_{0}, \Gamma_{\text {noise }}\right),
\end{gathered}
$$

onde $\Sigma$ é a variável aleatória Gaussiana que descreve a distribuição de condutividade $\sigma, V$ a variável aleatória Gaussiana que descreve a distribuição de tensão $v, E$ a variável aleatória Gaussiana que descreve o erro de medição $e, \sigma_{0}$ e $\Gamma_{p r}$ são a expectância e a covariância da distribuição de condutividade e $e_{0}$ e $\Gamma_{\text {noise }}$ a expectância e a covariância do erro de medição.

Quando é utilizado o modelo simplificado do problema direto $\tilde{f}($.) as distribuições estatísticas são alteradas. Neste caso a formulação do problema pode ser descrita por

$$
\begin{gathered}
v=\tilde{f}(\tilde{\sigma})+\tilde{e}, \\
V=\tilde{f}(\tilde{\Sigma})+\tilde{E} \\
\tilde{\sigma} \sim N\left(\tilde{\sigma}_{0}, \tilde{\Gamma}_{p r}\right) \quad \text { e } \tilde{e} \sim N\left(\tilde{e}_{0}, \tilde{\Gamma}_{\text {noise }}\right),
\end{gathered}
$$

onde $\tilde{\Sigma}$ é a variável aleatória Gaussiana que descreve a distribuição de condutividade $\tilde{\sigma}, \tilde{E}$ a variável aleatória Gaussiana que descreve o erro de medição $\tilde{e}_{,} \tilde{\sigma}_{0}$ e $\tilde{\Gamma}_{p r}$ são a expectância e a covariância da distribuição de condutividade para o modelo simplificado $\tilde{\sigma}, \tilde{e}_{0}$ e $\tilde{\Gamma}_{\text {noise }}$ são a expectância e a covariância do erro ẽ que descreve o erro de medição em conjunto com o erro de modelo.

Para encontrarmos a estatística do erro de aproximação soma-se e subtrai-se o modelo simplificado $\tilde{f}(\tilde{\Sigma})$ na equação 2.6 e posteriormente reordenam-se e agrupam-se alguns termos. Realizando este processo, equações 2.9 e 2.10, obtém-se o erro de apro- 
ximação $\tilde{E}$, mostrado na equação 2.8 e que pode ser descrito pela equação 2.11 .

$$
\begin{aligned}
& Y=f(\Sigma)+E+(\tilde{f}(\tilde{\Sigma})-\tilde{f}(\tilde{\Sigma})) \\
& Y=\tilde{f}(\tilde{\Sigma})+\underbrace{E+(f(\Sigma)-\tilde{f}(\tilde{\Sigma}))}_{\tilde{E}} \\
& \tilde{E}=E+(f(\Sigma)-\tilde{f}(\tilde{\Sigma}))
\end{aligned}
$$

Quando se utiliza o modelo simplificado sem nenhum tipo de correção assume-se que $E \approx \tilde{E}$ e consequentemente que o modelo simplificado adicionado de um erro de medição é suficiente para descrever o fenômeno. Para que não seja cometido este erro de modelagem é preciso levar em consideração a estatística do erro $\tilde{E}$ e assim aproximar o modelo simplificado do modelo mais acurado.

A partir da equação 2.11 podemos calcular a expectância e covariância do novo erro. Assumindo que $\Sigma$ e $E$ são variáveis não correlacionadas, o cálculo da expectância pode ser obtido por

$$
\begin{aligned}
\tilde{e}_{0} & =\mathbf{E}\{\tilde{E}\} \\
& =\mathbf{E}\{E+(f(\Sigma)-\tilde{f}(\tilde{\Sigma}))\} \\
& \approx e_{o}+f\left(\sigma_{0}\right)-\tilde{f}\left(\tilde{\sigma}_{0}\right) .
\end{aligned}
$$

Para o cálculo da covariância assume-se também que a redução do modelo pode ser descrita por um operador linear $P$, isto é,

$$
P: \mathbb{R}^{n_{r e f}} \rightarrow \mathbb{R}^{n_{\text {sim }}}, \quad \tilde{\sigma}=P \sigma
$$

onde $n_{r e f}$ é a dimensão da malha de elementos finitos do problema direto e $n_{\text {sim }}$ a dimensão da malha de elementos finitos do problema direto simplificado. A partir destas considerações e assumindo que o traço de $\Gamma_{\text {noise }}$ é dominante, Kaipio e Somersalo [7, p. 182] mostra que a matriz de covariância do erro $\tilde{E}$ pode ser aproximada por

$$
\tilde{\Gamma}_{\text {noise }} \approx \Gamma_{\text {noise }}
$$




\subsubsection{Modelos de eletrodo}

Existem diversos modelos numéricos para descrever a injeção de corrente no domínio. No modelo de eletrodo nodal, assume-se que toda a corrente é injetada em um único nó da malha de elementos finitos localizado na fronteira $\partial \Omega$. Este modelo superestima o potencial elétrico no nó de injeção e no seu entorno, pois obriga o modelo de elementos finitos concentrar toda a densidade de corrente imposta ao domínio através de um ponto infinitesimal. Outros efeitos também não são considerados por este modelo: a impedância de contato, o efeito shunt e o efeito de borda nos eletrodos.

Quando a corrente elétrica é injetada em um paciente por meio de eletrodos ocorre a transdução da corrente elétrica em corrente iônica na interface eletrodo-pele, esta conversão causa uma queda de potencial elétrico e pode ser modelada por meio de uma impedância de contato. Rosell et al. [18] mostrou que para aplicações clínicas a impedância de contato varia significativamente com a frequência da corrente de excitação. Vale ressaltar que a impedância de contato também pode variar entre eletrodos, isto é, quando comparada as impedâncias de dois eletrodos diferentes acoplados em um mesmo domínio espera-se que a impedância de contato varie.

O efeito shunt é caracterizado pela passagem de parte significativa da corrente injetada tangente à superfície do contorno, injetando parcela menor de corrente no interior do domínio. Devido a alta condutividade dos eletrodos, parte da corrente, ao invés de passar por dentro do meio, desloca-se próxima à superfície aonde estão alocados os eletrodos e utiliza-os como caminho de passagem, diminuindo assim a densidade de corrente no interior do domínio [19]. Outro efeito que também deve ser levado em consideração está relacionado ao comportamento da corrente na interface eletrodopele, onde a densidade de corrente fica concentrada em regiões da borda do eletrodo, esta característica é chamada de efeito de borda [9, Seç. 1.3].

Para a modelagem dos efeitos que ocorrem na interface do eletrodo com o domínio, Hua et al. [20] propõe, utilizando o MEF, o modelo de eletrodo completo para malhas 2D e, posteriormente, Vauhkonen et al. [21] apresenta um modelo similar para malhas 3D. A ideia do modelo proposto é representar cada eletrodo através de um elemento particular que se conecta à malha de elementos finitos do domínio e que reproduza os efeitos de queda de tensão, shunt e borda que ocorrem no eletrodo.

Vauhkonen et al. [21] propõe um modelo de interface dos eletrodos 3D por meio de 
um conjunto de elementos hexaédricos de faces paralelas, onde uma das faces deste conjunto esta conectada ao domínio e a outra face é curto-circuitada (nó virtual), Figura 2.1a. Neste modelo a corrente é injetada e a tensão é medida através da parte curto-circuitada que representa a face metálica da interface eletrodo-pele. A Figura 2.1 mostra graficamente como é realizado este processo, vale observar que a altura $t$ e a condutividade do elemento definem as características do comportamento do eletrodo. A formulação teórica e a implementação para MEF do complete electrode model $3 D$ pode ser encontrada em Vauhkonen et al. [21, Cap. 3].

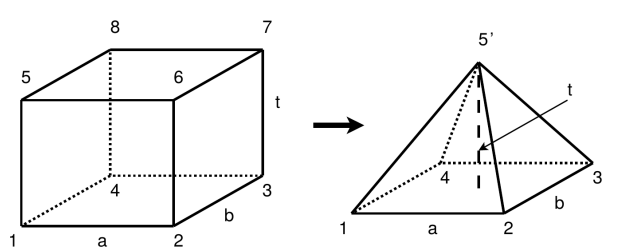

(a) Elemento hexaédrico: nós curtocircuitados

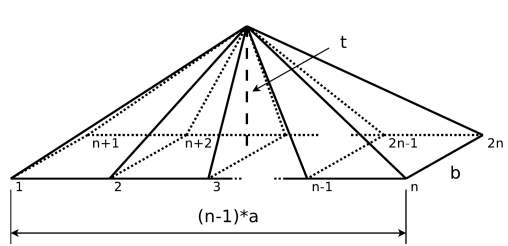

(b) Elemento eletrodo: nós curto-circuitados

Figura 2.1: Modelo de eletrodo completo 3D. FONTE: figuras extraídas de Moura [17, Seç. A.1.3]

Diferentes modelos de eletrodos foram apresentados e comparados em Silva [22], para o presente trabalho a implementação utilizada será o modelo prismático que é uma variação do complete electrode model que foi apresentado. A principal diferença está no elemento que representa o eletrodo, no modelo de eletrodo prismático utiliza-se um prisma de base triangular, pentaédrico, para substituir o elemento hexaédrico. Este modelo apresenta um comportamento próximo ao modelo Patacon ${ }^{6}$. A descrição do modelo formado por elementos prismáticos de base triangular pode ser encontrado em detalhes em Moura [17, Seç. A.1.3].

\subsubsection{Modelagem do efeito capacitivo nos eletrodos}

Na seção 2.1 foi descrito o tomógrafo ACE1, este tomógrafo é capaz de medir módulo e fase das tensões nos eletrodos e das correntes que são injetadas no domínio. Neste tomógrafo, devido a capacitância dos circuitos integrados de chaves analógicas e multiplexadores que compõem o active complex electrode, existe cerca de $56 \mathrm{pF}$ entre cada eletrodo e o terra, o que afeta os potenciais medidos nos eletrodos.

\footnotetext{
${ }^{6}$ Patacon é um modelo de eletrodo proposto em [22] que, segundo os critérios estabelecidos, apresentou os melhores resultados para descrever a distribuição de potencial no eletrodo.
} 
A TIE é dita um problema mal-posto e mal-condicionado, onde pequenas perturbações nas medidas podem provocar grandes artefatos nas imagens estimadas. Para mitigar o efeito da capacitância dos eletrodos na imagem modela-se este fenômeno por meio do MEF.

No modelo completo de eletrodo a matriz de condutividade global $[Y(\vec{\sigma})]$ (equação 2.3) é expandida, adiciona-se um nó virtual para cada eletrodo a ser modelado. Neste modelo, as correntes são injetadas por meio destes nós virtuais e assume-se que as tensões nos eletrodos são as tensões calculadas nestes nós.

Para levar em consideração o efeito capacitivo, é incorporado um capacitor entre cada um dos nós virtuais do modelo completo de eletrodo e o nó terra da malha. Estes capacitores podem ser modelados por meio de um elemento concentrado e sua matriz local pode ser calculada por

$$
Y_{\text {local }}^{\text {cap }}=\frac{1}{Z_{c}}\left[\begin{array}{rr}
1 & -1 \\
-1 & 1
\end{array}\right] \text {, para } Z_{c}=\frac{1}{j(2 \pi f) C},
$$

onde $Z_{c}$ é a impedância do capacitor, $f$ é a frequência do sinal de excitação, $C$ o valor de capacitância do eletrodo de medição e $j$ a unidade imaginária. 


\subsection{Solução do problema inverso}

Em TIE o problema inverso consiste em estimar a distribuição de condutividade interna de um domínio, dado os potenciais elétricos medidos na sua fronteira e as correntes elétricas aplicadas. Este problema pode ser equacionado como um problema de minimização, onde procura-se minimizar uma função objetivo, cujo o mínimo desta função resultaria em uma boa estimativa da distribuição de condutividade utilizada para descrever o domínio. Sob este o ponto de vista, diferentes métodos numéricos podem ser aplicados e o método dos mínimos quadrados é comumente utilizado para a construção da função objetivo.

Utilizando o método dos mínimos quadrados procura-se estimar o vetor de condutividade dos elementos da malha de elementos finitos $\vec{\sigma}$ que minimize a diferença entre o conjunto de tensões medidas através dos eletrodos $\vec{\phi}_{m} \in \mathbb{C}^{(n e . n p) \times 1}$ e as tensões calculadas por meio do problema direto $\vec{\phi}_{c}(\vec{\sigma}) \in \mathbb{C}^{(n e . n p) \times 1}$,

$$
r_{1}(\vec{\sigma})=\frac{1}{2}\left(\vec{\phi}_{m}-\vec{\phi}_{c}(\vec{\sigma})\right)^{t}\left(\vec{\phi}_{m}-\vec{\phi}_{c}(\vec{\sigma})\right)
$$

Por se tratar de uma problema não linear e mal-posto [6, 8], a solução não é estável e possui infinitas soluções possíveis. Para que o problema admita solução única e seja menos influenciado por erros de medição, utiliza-se o método de regularização de Tikhonov generalizado, que esta descrito em Vauhkonen [4, Seç. 2.3.4]. Este método propõe a adição de mais um termo na equação 2.18 e incorpora informações adicionais acerca do domínio, transformando o problema em bem posto. A equação 2.19 mostra a nova função objetivo,

$$
r_{2}(\vec{\sigma})=\left(\vec{\phi}_{m}-\vec{\phi}_{c}(\vec{\sigma})\right)^{t}[I]\left(\vec{\phi}_{m}-\vec{\phi}_{c}(\vec{\sigma})\right)+\lambda^{2}\left(\vec{\sigma}-\vec{\sigma}^{*}\right)^{t}[L]^{t}[L]\left(\vec{\sigma}-\vec{\sigma}^{*}\right)
$$

onde $[L] \in \mathbb{R}^{m \times m}$ é uma matriz de regularização do problema, $[I] \in \mathbb{R}^{\left(n_{e} \cdot n_{p}\right) \times\left(n_{e} . n_{p}\right)}$ uma matriz identidade, $\vec{\sigma}^{*} \in \mathbb{C}^{m}$ o vetor de distribuição de condutividade esperada na malha de elementos finitos e $\lambda$ o parâmetro de regularização que controla o peso da regularização adotada. 


\subsubsection{Método de Gauss-Newton}

O método de Gauss-Newton (GN) é um método iterativo utilizado para solução de problemas de mínimos quadrados não lineares. Como característica principal este método realiza uma aproximação da matriz Hessiana, matriz que descreve as derivadas parciais de segunda ordem da função vetorial a ser minimizada, utilizando apenas as derivadas parciais de primeira ordem desta função. Mais detalhes sobre a computação das matrizes envolvidas, análise de convergência e desempenho do Método de Gauss-Newton podem ser encontrados em Björck [23] e Vauhkonen [4].

Seja a equação 2.19 a função objetivo a ser minimizada, Vauhkonen [4] mostra que as iterações do método de GN podem ser calculadas por

$$
\begin{aligned}
\vec{\sigma}_{i+1} & =\vec{\sigma}_{i}+\alpha_{i} \cdot \Delta \vec{\sigma}_{i} \\
\Delta \vec{\sigma}_{i} & =\left(\left[J_{i}\right]^{t}\left[J_{i}\right]+\lambda^{2}[L]^{t}[L]\right)^{-1}\left(\left[J_{i}\right]^{t}\left(\vec{\phi}_{m}-\vec{\phi}_{c}\left(\vec{\sigma}_{i}\right)\right)-\lambda^{2}[L]^{t}[L]\left(\vec{\sigma}_{i}-\vec{\sigma}^{*}\right)\right),
\end{aligned}
$$

onde $\left[J_{i}\right] \in \mathbb{C}^{\left(n_{e} \cdot n_{p}\right) \times m}$ é a matriz Jacobiana de $\vec{\phi}_{c}\left(\vec{\sigma}_{i}\right)$ e $\alpha_{i}$ o tamanho do passo na iteração $i$.

\section{Estimativa inicial}

Para que o método de Gauss-Newton apresente bom desempenho é necessário que a estimativa inicial esteja próxima da solução. Uma forma de contornar esta fragilidade é estimar primeiramente as condições iniciais para que o vetor $\sigma$ fique o mais próximo possível da solução do problema.

Esta etapa pode ser realizada fazendo uma estimativa inicial de apenas 3 parâmetros através do método GN, a partir de $\vec{\phi}_{m}$, a saber: a condutividade média do meio (domínio) $\left(\sigma_{\text {meio }}\right)$, a impedância de contato média do modelo de eletrodo $\left(\sigma_{\text {imp_cont }}\right)$ e o efeito capacitivo médio do eletrodo $\left(Z_{C_{\text {media }}}\right)$.

Nesta estimativa inicial a matriz Jacobiana $J$ também seria calculada em relação a estas 3 incógnitas e a matriz de regularização $L$ pode ser diagonal contendo o inverso da variância esperada dos 3 parâmetros envolvidos. 


\subsubsection{Método D-bar}

O método $D$-bar pertence a uma classe de métodos diretos utilizados para a solução de problemas inversos não lineares. Estes métodos são derivados da teoria de inverse scattering [6] e estimam a solução do problema inverso por meio da Complex Geometrical Optics solutions ${ }^{7}$. Como exemplo de aplicação, o método D-bar tem sido utilizado para o monitoramento das funções pulmonares e identificação de regiões com aprisionamento de ar em pacientes com fibrose cística [25, 26].

No presente trabalho é utilizado uma variação do método D-bar descrito em Murphy e Mueller [27], que estima a condutividade do domínio não-circular utilizando a solução exponencial crescente de Faddeev para resolver a equação de Schrödinger. Esta variação é baseada em Isaacson et al. [28] e simplificadamente pode ser descrita através das seguintes etapas,

$$
\left.\left(\Lambda_{\sigma}-\Lambda_{r e f}\right) \rightarrow \psi(., k)\right|_{\partial \Omega} \rightarrow t^{e x p}(k) \rightarrow u\left(z_{x y}, k\right) \rightarrow \sigma
$$

Na primeira etapa estima-se os parâmetros $\Lambda_{\sigma}$ e $\Lambda_{\text {ref }}$ a partir das tensões medidas $V_{\sigma}$ e $V_{\text {ref }}$. Estas variáveis representam o mapa de tensão na fronteira para distribuição de densidade de corrente (DN map). Quando a tensão $V_{\text {ref }}$ utilizada para calcular $\Lambda_{\text {ref }}$ deriva de uma tensão medida a imagem estimada é denominada imagem relativa e quando esta tensão é calcula por meio de um modelo computacional a imagem é considerada uma imagem absoluta.

Em um segundo momento determina-se a solução de Faddeev $\left.\psi(., k)\right|_{\partial \Omega}$ para a equação de Schrödinger. Posteriormente calcula-se a scattering transform $t^{\exp }(k)$ considerando a posição dos eletrodos no plano complexo. Em Murphy e Mueller [27] é mostrado como pode ser calculado $t^{e x p}(k)$ para um domínio não-circular.

Após o cálculo da transformada $t^{e x p}(k)$ um raio de truncamento é escolhido e os valores no plano complexo maiores que este raio são zerados. O truncamento da scattering transform pode ser interpretado como uma regularização e os seus efeitos foram estudados em [29].

Uma outra opção é usar dois raios de truncamento $R_{1}$ e $R_{2}$, para $t^{\exp }\left(|k| \leq R_{1}\right)$ todos

\footnotetext{
${ }^{7}$ Também chamadas de soluções exponenciais crescentes, esta ferramenta é crítica para a elaboração do método. A descrição, focada na TIE, deste conjunto de soluções pode ser encontrada em detalhes em Mueller e Siltanen [6, Cap. 14] e Siltanen et al. [24].
} 
os pontos são considerados e para $t^{\exp }\left(|k|>R_{2}\right)$ todos os pontos são zerados. Para os pontos intermediários $t^{\exp }\left(R_{1}<|k| \leq R_{2}\right)$, apenas os pontos cuja amplitude seja menor que a máxima amplitude encontrada em $t^{\exp }\left(|k| \leq R_{1}\right)$ são considerados.

A partir da scattering transform truncada é resolvido a equação D-bar, [27, Eq. 4] para $u\left(z_{x y}, k\right)$ em cada ponto $z_{x y}$ na região de interesse do plano complexo. A solução numérica para a equação D-bar pode ser encontrada em detalhes em Mueller e Siltanen [6, Cap. 15]. E como último passo a estimativa de $\sigma$ em cada ponto de interesse $z_{x y}$ é obtida a partir da solução $u^{2}\left(z_{x y}, k=0\right)$.

Para mais especificações sobre o método utilizado neste trabalho Murphy e Mueller [27] descrevem-o em detalhe. Uma visão geral sobre a classe de algoritmos D-bar e suas aplicações pode ser encontrada em Mueller e Siltanen [6].

\subsection{Informação a priori}

O problema de TIE é dito mal-posto, uma forma de contornar o problema é através da inclusão de conhecimento a priori sobre a distribuição de condutividade que se deseja estimar. Sob o ponto de vista probabilístico, o problema de TIE pode ser interpretado como a estimação da distribuição de condutividade $\Sigma$ através do critério de máxima probabilidade a posteriori (MAP), mostrado na equação 2.21. Para isto, assume-se que as distribuições de potencial e de condutividade do domínio são variáveis aleatórias. Para métodos iterativos, a introdução de priors tem sido utilizada para condicionar o problema de TIE e melhorar a resolução espacial das imagens $[8,30,31,32,33,34,35,36,37]$.

$$
\Sigma_{M A P}=\underset{\sigma}{\operatorname{argmax}} \pi\left(\sigma \mid \vec{\phi}_{m}\right)
$$

onde $\pi\left(\sigma \mid \vec{\phi}_{m}\right)$ é a função densidade de probabilidade (fdp) de $\sigma$ dado o evento observado $\vec{\phi}_{m}$, ou seja essa variável expressa a probabilidade de que a distribuição de condutividade seja uma distribuição particular $\sigma$ dado que foi realizado um conjunto de medidas $\vec{\phi}_{m}$.

Na seção 2.3 foi descrito que a TIE é considerado um problema mal-posto. Uma forma de tentar reduzir o desconhecimento acerca do sistema é levar em considera- 
ção informações adicionais através das probabilidades associadas às variáveis que o compõe. Para explicitar as probabilidades marginais, aplica-se o teorema de Bayes à função densidade de probabilidade $\pi\left(\sigma \mid \vec{\phi}_{m}\right)$,

$$
\pi\left(\sigma \mid \vec{\phi}_{m}\right)=\frac{\pi\left(\vec{\phi}_{m} \mid \sigma\right) \cdot \pi(\sigma)}{\pi\left(\vec{\phi}_{m}\right)}
$$

onde $\pi\left(\vec{\phi}_{m} \mid \sigma\right)$ é a função de verossimilhança, $\pi(\sigma)$ a função densidade de probabilidade a priori de $\sigma$ e $\pi\left(\vec{\phi}_{m}\right)$ a verossimilhança marginal.

Segundo Kaipio e Somersalo [7, Seç. 3.4] quando a fdp é calculada a partir de um grande número de amostras de eventos aleatórios e mutuamente independentes as densidades Gaussianas são boas aproximações mesmo para distribuições nãoGaussianas. Assumindo que a variável aleatória que representa a distribuição de $\sigma$ é obtida por amostragem ela pode ser considerada Gaussiana e a sua fdp a priori é descrita por

$$
\pi(\sigma) \propto \exp \left(-\frac{1}{2}\left(\sigma-\sigma_{0}\right)^{t} \Gamma_{p r}^{-1}\left(\sigma-\sigma_{0}\right)\right)
$$

A função de verossimilhança apresenta a probabilidade de um evento $\vec{\phi}_{m}$ acontecer (realização) dado uma distribuição de condutividade $\sigma$ (estado). Assumindo que o erro de medida e é Gaussiano, a função de verossimilhança é calculada por

$$
\pi\left(\vec{\phi}_{m} \mid \sigma\right) \propto \exp \left(-\frac{1}{2}\left(\vec{\phi}_{m}-f(\sigma)-e_{0}\right)^{t} \Gamma_{\text {noise }}^{-1}\left(\vec{\phi}_{m}-f(\sigma)-e_{0}\right)\right)
$$

Finalmente, o termo $\pi\left(\vec{\phi}_{m}\right)$ da equação 2.22 é aproximadamente uma constante, não nula, e neste caso, não tem importância para TIE [38]. Deste modo, a função densidade de probabilidade $\pi\left(\sigma \mid \vec{\phi}_{m}\right)$ pode ser reescrita como

$$
\begin{aligned}
\pi\left(\sigma \mid \vec{\phi}_{m}\right) \propto & \exp \left(-\frac{1}{2}\left(\vec{\phi}_{m}-f(\sigma)-e_{0}\right)^{t} \Gamma_{\text {noise }}^{-1}\left(\vec{\phi}_{m}-f(\sigma)-e_{0}\right)\right) \times \\
& \exp \left(-\frac{1}{2}\left(\sigma-\sigma_{0}\right)^{t} \Gamma_{p r}^{-1}\left(\sigma-\sigma_{0}\right)\right)
\end{aligned}
$$

Aplicando o logaritmo natural e eliminando as constantes multiplicativas pode-se definir a função de minimização, equação 2.28. Esta função objetivo tem a mesma 
forma da função objetivo mostrada na equação 2.19 e são equivalentes quando $\Gamma_{n o i s e}^{-1}$ é a matriz identidade, $\vec{\phi}_{c}=f(\sigma)+e_{0}, \lambda=1,[L]=\sqrt{\Gamma_{p r}^{-1}}$ e $\sigma_{0}=\vec{\sigma}^{*}$.

$$
\begin{aligned}
\Sigma_{M A P} & =\underset{\sigma}{\operatorname{argmax}} \pi\left(\sigma \mid \vec{\phi}_{m}\right) \\
& =\underset{\sigma}{\operatorname{argmin}}\left(-\ln \pi\left(\sigma \mid \vec{\phi}_{m}\right)\right) \\
& =\underset{\sigma}{\operatorname{argmin}}\left(\left(\vec{\phi}_{m}-f(\sigma)-e_{0}\right)^{t} \Gamma_{\text {noise }}^{-1}\left(\vec{\phi}_{m}-f(\sigma)-e_{0}\right)+\left(\sigma-\sigma_{0}\right)^{t} \Gamma_{p r}^{-1}\left(\sigma-\sigma_{0}\right)\right)
\end{aligned}
$$

Uma forma recorrente utilizada na TIE para acrescentar informação ao problema é utilizar como matriz de regularização $L$ (equação 2.19) um filtro Gaussiano espacial passa-altas $(F)$. Neste caso, soluções de alta frequência espacial são penalizadas, privilegiando a escolha de distribuições de condutividades mais suaves.

Caso a matriz de regularização $L$ seja uma matriz identidade a função objetivo prioriza soluções próximas de $\vec{\sigma}^{*}$. Outra forma de adicionar informação a priori no problema é utilizar funções densidade de probabilidade baseadas em conjuntos de amostras.

Conforme apresentado na Seção 2.2.3, a utilização de modelos simplificados muitas vezes se faz necessária para a estimação das distribuições de condutividade. Como último passo, a teoria do erro de aproximação pode ser aplicada na equação 2.28,

$$
\begin{aligned}
\Sigma_{M A P} & =\underset{\tilde{\sigma}}{\operatorname{argmin}}\left(\left(\vec{\phi}_{m}-\tilde{f}(\tilde{\sigma})-\tilde{e}_{0}\right)^{t} \tilde{\Gamma}_{\text {noise }}^{-1}\left(\vec{\phi}_{m}-\tilde{f}(\tilde{\sigma})-\tilde{e}_{0}\right)+\left(\tilde{\sigma}-\tilde{\sigma}_{0}\right)^{t} \tilde{\Gamma}_{p r}^{-1}\left(\tilde{\sigma}-\tilde{\sigma}_{0}\right)\right), \\
\tilde{\sigma} & \Rightarrow\left\{\begin{array}{l}
\tilde{\sigma}_{0}=P . \sigma_{0} \\
\tilde{\Gamma}_{p r}=P \Gamma_{p r} P^{t}
\end{array} \quad, \quad \tilde{e} \Rightarrow\left\{\begin{array}{l}
\tilde{e}_{0}=e_{0}+f\left(\sigma_{0}\right)-\tilde{f}\left(\tilde{\sigma}_{0}\right) \\
\tilde{\Gamma}_{\text {noise }} \approx \Gamma_{\text {noise }}
\end{array} .\right.\right.
\end{aligned}
$$

\subsubsection{Atlas anatômico de tórax suíno}

Em seu trabalho, Camargo [8] desenvolve, calcula e testa um prior estatístico baseado em amostras de tórax suíno. Neste estudo, informações fisiológicas e anatômicas 
coletadas in vivo foram utilizadas para a construção de um atlas anatômico ${ }^{8}$.

Imagens de tomografia computadorizada foram segmentadas em 5 regiões distintas (músculo, pulmão aerado, atelectasia, coração e osso), registradas e normalizadas. Para cada tecido foi medida a resistividade no tempo e in vivo [39]. A partir deste conjunto de amostras foi estimado a distribuição média de resistividade $\left(\rho_{0_{s w}}\right)$ e sua respectiva matriz de covariância $\left(\Gamma_{s w}\right)$.

Sob a ótica de regularizar um problema inverso mal-posto, Camargo [8] inclui o atlas anatômico desenvolvido como regularização adicional na função objetivo $r_{2}(\vec{\rho})$ (equação 2.19),

$$
\begin{aligned}
r_{3}(\vec{\rho}) & =\left(\vec{\phi}_{m}-\vec{\phi}_{c}(\vec{\rho})\right)^{t}[I]\left(\vec{\phi}_{m}-\vec{\phi}_{c}(\vec{\rho})\right)+\lambda^{2}\left(\vec{\rho}-\vec{\rho}^{*}\right)^{t}[L]^{t}[L]\left(\vec{\rho}-\vec{\rho}^{*}\right) \\
& +\gamma^{2}\left(\vec{\rho}-\vec{\rho}_{0 w}\right)^{t} \Gamma_{s w}^{-1}\left(\vec{\rho}-\vec{\rho}_{0 w}\right)
\end{aligned}
$$

onde é utilizado um filtro gaussiano espacial passa-altas $F$ como matriz de regularização $L, \vec{\rho}^{*}$ é a resistividade esperada e $\gamma$ é o parâmetro de regularização associado ao atlas anatômico.

Estimando apenas a parte real da distribuição de impeditividade do modelo simplificado e acrescentando o erro de aproximação, Camargo [8] calcula, pelo método de Gauss-Newton, a distribuição de resistividade de um tórax suíno por meio da minimização da função objetivo $r(\vec{\rho})$,

$$
\begin{aligned}
r(\vec{\rho}) & =\left(\vec{\phi}_{m}-\vec{\phi}_{c}(\vec{\rho})\right)^{t}[I]\left(\vec{\phi}_{m}-\vec{\phi}_{c}(\vec{\rho})\right)+\lambda^{2}\left(\vec{\rho}-\vec{\rho}^{*}\right)^{t}[F]^{t}[F]\left(\vec{\rho}-\vec{\rho}^{*}\right) \\
& +\gamma^{2}\left(\vec{\rho}-{\overrightarrow{\rho_{0}}}_{s w}\right)^{t} \Gamma_{s w}^{-1}\left(\vec{\rho}-{\overrightarrow{\rho_{0}}}_{s w}\right) .
\end{aligned}
$$

\subsubsection{Informação a priori no método $D$-bar}

A técnica de introduzir informações a priori por meio da adição de um termo de penalização à função objetivo, como é feito no método de Gauss-Newton, não pode ser generalizada para o método D-bar. Métodos diretos que levam em consideração informações anatômicas e fisiológicas na reconstrução da condutividade são pouco explorados.

Alsaker e Mueller [40] propuseram o primeiro método de reconstrução direto para

\footnotetext{
${ }^{8}$ Em aplicações de imageamento médico, o termo atlas anatômico é usado para descrever informações a priori baseadas em amostras que contém informação anatômica e fisiológica [7, Seç. 3.3.5].
} 
TIE que incorpora informações a priori na reconstrução. Neste trabalho é implementado um método $D$-bar onde a distribuição de condutividade esperada é levada em consideração na scattering transform. Durante esta etapa do processo a scattering transform é truncada utilizando diferentes raios para a parte da medida $t(k)$ e para a parte da informação a priori $t_{p r}(k)$ [40, Eq.(3.6)].

\subsection{Complemento de Schur}

O complemento de Schur é uma propriedade matricial que correlaciona blocos de uma matriz simétrica positiva definida. Esta relação é utilizada em diversas áreas da engenharia e matemática, destacando-se principalmente no campo dos métodos numéricos. Na área de probabilidade e estatística o complemento de Schur tem papel importante como ferramenta para o cálculo de médias e covariâncias condicionais [41, 42, 43].

Seja $\Gamma \in \mathbb{R}^{n \times n}$ uma matriz simétrica positiva definida dividida em blocos,

$$
\Gamma=\left[\begin{array}{ll}
\Gamma_{11} & \Gamma_{12} \\
\Gamma_{21} & \Gamma_{22}
\end{array}\right]
$$

onde $\Gamma_{11} \in \mathbb{R}^{k \times k}, \Gamma_{22} \in \mathbb{R}^{(n-k) \times(n-k)}, \Gamma_{21}=\Gamma_{12}{ }^{t}$ e $k<n$. O complemento de Schur desta matriz pode ser definido como

$$
\begin{aligned}
& \tilde{\Gamma}_{22}=\Gamma_{11}-\Gamma_{12} \Gamma_{22}{ }^{-1} \Gamma_{21} \quad \mathrm{e} \\
& \tilde{\Gamma}_{11}=\Gamma_{22}-\Gamma_{21} \Gamma_{11}{ }^{-1} \Gamma_{12} .
\end{aligned}
$$

Quando as matrizes $\Gamma_{11}, \Gamma_{22}, \tilde{\Gamma}_{11}$ e $\tilde{\Gamma}_{22}$ são inversíveis, a matriz $\Gamma$ também é inversível e pode ser calculada como mostrado na equação $2.34^{9}$.

$$
\Gamma^{-1}=\left[\begin{array}{cc}
\tilde{\Gamma}_{22}^{-1} & -\tilde{\Gamma}_{22}^{-1} \Gamma_{12} \Gamma_{22}{ }^{-1} \\
-\tilde{\Gamma}_{22}^{-1} \Gamma_{12} \Gamma_{22}{ }^{-1} & \tilde{\Gamma}_{11}^{-1}
\end{array}\right]
$$

\footnotetext{
${ }^{9}$ Apêndice A.1 descreve como esta propriedade matricial pode ser obtida.
} 


\subsubsection{Propriedade do complemento de Schur para distribuições Gaussianas}

O teorema 3.5 descrito em [7, Seç. 3.4] ${ }^{10}$. mostra que a partir da densidade de probabilidade conjunta $\pi(x, y): \mathbb{R}^{n} \times \mathbb{R}^{k} \rightarrow \mathbb{R}_{+}$de duas variáveis aleatórias Gaussianas $X: \Omega \rightarrow \mathbb{R}^{n}$ e $Y: \Omega \rightarrow \mathbb{R}^{k}$,

$$
\pi(x, y) \propto \exp \left(-\frac{1}{2}\left[\begin{array}{l}
x-x_{0} \\
y-y_{0}
\end{array}\right]^{t}\left[\begin{array}{c}
\Gamma_{x x} \Gamma_{x y} \\
\Gamma_{y x} \Gamma_{y y}
\end{array}\right]^{-1}\left[\begin{array}{c}
x-x_{0} \\
y-y_{0}
\end{array}\right]\right)
$$

a distribuição de probabilidade condicional de $X$ dado $Y=y, \pi(x \mid y): \mathbb{R}^{n} \rightarrow \mathbb{R}_{+}$, pode ser escrita por,

$$
\begin{aligned}
\pi(x \mid y) & \propto \exp \left(-\frac{1}{2}(x-\bar{x})^{t} \tilde{\Gamma}_{y y}^{-1}(x-\bar{x})\right), \\
\tilde{\Gamma}_{y y} & =\Gamma_{x x}-\Gamma_{x y} \Gamma_{y y}{ }^{-1} \Gamma_{y x} \mathrm{e} \\
\bar{x} & =x_{0}+\Gamma_{x y} \Gamma_{y y}{ }^{-1}\left(y-y_{0}\right),
\end{aligned}
$$

onde $\Gamma_{x x}$ e $\Gamma_{y y}$ representam as matrizes de covariância das variáveis aleatórias Gaussianas $x$ e $y, \Gamma_{x y}$ e $\Gamma_{y x}$ as suas matrizes de covariância cruzadas e $x_{o}$ e $y_{0}$ os seus valores esperados.

\subsubsection{Estimativa do máximo a posteriori}

Conhecida a distribuição de probabilidade condicional de $X$ dado $Y=y$, equação 2.36, temos que a distribuição do máximo a posteriori de $x$ pode ser calculada por,

$$
X_{\text {map }}=\underset{x}{\operatorname{argmax}}(\pi(x \mid y)) .
$$

Aplicando o logaritmo natural e eliminando as constantes multiplicativas, a função de maximização descrita na equação 2.37 pode ser reescrita por meio de uma função de minimização equivalente,

$$
X_{\text {map }}=\underset{x}{\operatorname{argmin}}\left((x-\bar{x})^{t} \tilde{\Gamma}_{y y}^{-1}(x-\bar{x})\right) .
$$

${ }^{10} \mathrm{~A}$ demonstração e as considerações para a utilização do teorema 3.5 apresentada em Kaipio e Somersalo [7, Seç. 3.4] estão descritas no Apêndice A.2. 
Utilizando as propriedades matriciais da covariância $\tilde{\Gamma}_{y y}{ }^{11}$ é possível mostrar que $\bar{x}$ representa a distribuição que maximiza a distribuição a posteriori,

$$
\begin{aligned}
X_{\text {map }} & =\underset{x}{\operatorname{argmin}}\left((x-\bar{x})^{t} \tilde{\Gamma}_{y y}^{-1}(x-\bar{x})\right) \\
& =\bar{x} \\
& =x_{0}+\Gamma_{x y} \Gamma_{y y}{ }^{-1}\left(y-y_{0}\right) .
\end{aligned}
$$

${ }^{11}$ A demonstração e as considerações para o cálculo da estimativa do máximo a posteriori a partir das propriedades do complemento de Schur estão descritas no Apêndice A.3 


\section{Capítulo 3}

\section{Método}

Na teoria probabilística, a construção e aplicação de funções de densidade de probabilidade a priori para a solução de problemas inversos tem obtido grande sucesso [7]. Abordado na Seção 2.4.1, Camargo [8] mostra em seu trabalho que a utilização de um atlas anatômico em conjunto com a teoria do erro de aproximação melhora significativamente a resolução espacial de imagens absolutas de tórax.

Como visto na Seção 2.3.2, através do método D-bar não é possível utilizar o mesmo enfoque e incorporar informação a priori por meio de uma penalização adicional, como é realizado no método de Gauss-Newton. Devido a forma com que o método D-bar estima a distribuição de condutividade a inclusão de priors na formulação do problema torna-se não evidente.

Neste contexto, é proposto nesta seção duas diferentes formas de introduzir informações a priori e minimizar erros de modelagem no método $D$-bar:

\section{Introdução de informação $a$ priori através do ajuste da tensão:}

O método consiste em ajustar previamente as tensões que são utilizadas na estimação da imagem no método $D$-bar. Procura-se minimizar uma função objetivo que considera erros de modelo e inclui uma função densidade de probabilidade com informações anatômicas e fisiológicas acerca do domínio avaliado.

2. Introdução de informação a priori através do pós-processamento das imagens reconstruídas

O método consiste no pós-processamento das imagens já reconstruídas pelo método $D$-bar. Sob a ótica Bayesiana, aplicam-se as propriedades do complemento 
de Schur para estimar a densidade de probabilidade condicional que calcule o valor esperado (e mais acurado) de uma distribuição de condutividade quando conhecida a distribuição de condutividade estimada pelo algoritmo.

Por meio de testes numéricos e fantomas experimentais ${ }^{12}$ avalia-se o desempenho dos dois métodos propostos no algoritmo de reconstrução $D$-bar. Testes para a validação e avaliação dos métodos são propostos na Seção 3.3.

\subsection{Introdução de informação a priori através do ajuste}

\section{da tensão}

Pelo método do erro de aproximação descrito na Seção 2.2.3 sabe-se que é necessário corrigir o problema inverso, mais especificamente, o modelo de ruído. Uma aproximação da correção do modelo de ruído consiste em adicionar o valor esperado dos erros entre modelo não simplificado e modelo simplificado ao vetor de tensões calculado pelo modelo simplificado. Isto equivale algebricamente a pré-condicionar as medidas,

$$
\begin{aligned}
& v=\tilde{f}(\tilde{\sigma})+\underbrace{\left(e_{0}+f\left(\sigma_{0}\right)-\tilde{f}\left(\tilde{\sigma_{0}}\right)\right)}_{\tilde{e}_{0}}, \\
& v=\tilde{f}\left(\tilde{\sigma_{0}}\right)+\tilde{e}_{0}, \\
& \underbrace{v-\tilde{e}_{0}}_{\text {pré-cond. da medida }}=\tilde{f}\left(\tilde{\sigma_{0}}\right) .
\end{aligned}
$$

Ampliando agora o conceito do erro de aproximação, propõe-se pré-condicionar a medida levando em consideração não apenas o valor esperado do erro de aproximação, mas também a função densidade de probabilidade a priori de um conjunto de medidas $v$ acontecer. Formulando o problema deste modo, procura-se então estimar uma distribuição de tensão $V_{M A P}$ que maximize a densidade de probabilidade a posteriori dado o conjunto de medidas $v$,

\footnotetext{
${ }^{12}$ Fantoma experimental é um termo utilizado na TIE para descrever ensaios onde objetos com geometria e propriedades definidas são avaliados em recipientes com condições controladas de forma que somente algumas condições específicas encontradas em testes reais sejam observadas.
} 


$$
V_{M A P}=\underset{v_{c}}{\operatorname{argmax}} \pi\left(v_{c} \mid v\right)
$$

onde $v_{c}$ são as medidas pré-condicionadas a serem estimadas.

\subsubsection{Formulação probabilística para estimação das medidas}

Retomando a teoria do erro de aproximação, foi mostrado que corrigir a problema inverso inclui uma etapa que equivale a condicionar as medidas. Vamos denotar as medidas condicionadas por $v_{c}$ e denotar as medidas físicas, propriamente ditas, por $v$. Vamos adotar um modelo simples e linear entre $v$ e $v_{c}$, equação 3.2. A tensão medida $v$ é igual à tensão $v_{c}$, que pode ser vista como a solução de um problema direto simplificado $\tilde{f}(\tilde{\sigma})$, acrescida do erro de aproximação $(\tilde{e})$.

$$
v=\tilde{f}(\tilde{\sigma})+\tilde{e}
$$

Implicitamente o método D-bar utiliza um modelo da realidade física em que alguns aspectos da eletrônica de medição e do domínio estão ausentes, a capacitância parasita, a impedância de contato, domínio físico em 3D, por citar alguns. O modelo que calcula $v_{c}$ deve conter as mesmas simplificações, equação 3.3.

$$
v_{c}=\tilde{f}(\tilde{\sigma})
$$

Admite-se que a distribuição de condutividade a priori, também chamada de Atlas Anatômico, pode ser considerada um vetor de variáveis aleatórias Gaussianas, equação 3.4 ,

$$
\tilde{\sigma} \sim N\left(\tilde{\sigma}_{0}, \tilde{\Gamma}_{p r}\right)
$$

onde $\tilde{\sigma_{0}}$ e $\tilde{\Gamma}_{p r}$ são a expectância e a covariância do conjunto amostrado de distribuições de condutividade $\tilde{\sigma}$. 
As amostras utilizadas para calcular a equação 3.4, $\tilde{\sigma}_{i}$ podem ser transformadas em amostras de tensões, $v_{c_{i}}$, através de um modelo de elementos finitos que contenha as mesmas simplificações implícitas no método $D$-bar, gerando uma nova distribuição em tensões, equação 3.5 .

$$
v_{c} \sim N\left(\tilde{v}_{0}, \tilde{\Gamma}_{p r_{v}}\right)
$$

onde $\tilde{v}_{0}$ e $\tilde{\Gamma}_{p r_{v}}$ são a expectância e a covariância do conjunto de tensões calculados $v_{c}$ a partir do conjunto amostrado de distribuições de condutividade $\tilde{\sigma}$. Esta distribuição de probabilidade será chamada de Atlas de Tensões. Observa-se que ela contém informação anatômica, fisiológica e de eletrônica.

Uma vez conhecida a estatística de $v_{c}$ e o modelo determinístico de $v$, aplica-se o teorema de Bayes para função densidade de probabilidade $\pi\left(v_{c} \mid v\right)$,

$$
\pi\left(v_{c} \mid v\right)=\frac{\pi\left(v \mid v_{c}\right) \cdot \pi\left(v_{c}\right)}{\pi(v)}
$$

onde $\pi\left(v \mid v_{c}\right)$ é a função de verossimilhança, $\pi\left(v_{c}\right)$ a fdp a priori de $v_{c}$ e $\pi(v)$ a verossimilhança marginal.

Utilizando os conceitos mostrados na Seção 2.4, as funções densidade de probabilidade da equação 3.6 podem ser calculadas por,

$$
\begin{aligned}
\pi\left(v \mid v_{c}\right) & \propto \exp \left(-\frac{1}{2}\left(v-v_{c}-\tilde{e}_{0}\right)^{T} \tilde{\Gamma}_{\text {noise }}^{-1}\left(v-v_{c}-\tilde{e}_{0}\right)\right) \\
\pi\left(v_{c}\right) & \propto \exp \left(-\frac{1}{2}\left(v_{c}-\tilde{v}_{0}\right)^{T} \tilde{\Gamma}_{p r_{v}}^{-1}\left(v_{c}-\tilde{v}_{0}\right)\right) \mathrm{e} \\
\pi(v) & \approx \text { cte. }
\end{aligned}
$$

Aplicando o logaritmo natural e eliminando as constantes multiplicativas pode-se 
definir a função de minimização,

$$
\begin{aligned}
V_{M A P} & =\underset{v_{c}}{\operatorname{argmax}} \pi\left(v_{c} \mid v\right) \\
& =\underset{v_{c}}{\operatorname{argmin}}\left(-\ln \pi\left(v_{c} \mid v\right)\right) \\
& =\underset{v_{c}}{\operatorname{argmin}}\left(\left(v-v_{c}-\tilde{e}_{0}\right)^{T} \tilde{\Gamma}_{n o i s e}^{-1}\left(v-v_{c}-\tilde{e}_{0}\right)+\left(v_{c}-\tilde{v}_{0}\right)^{T} \tilde{\Gamma}_{p r_{v}}^{-1}\left(v_{c}-\tilde{v}_{0}\right)\right)
\end{aligned}
$$

Do ponto de vista prático, a matriz de covariância $\Gamma_{p r_{v}}$ não é bem conhecida, assim torna-se necessário utilizar um parâmetro adicional $\beta$ para controlar o peso relativo entre verossimilhança e informação a priori,

$$
V_{M A P}=\underset{v_{c}}{\operatorname{argmin}}\left(\left(v-v_{c}-\tilde{e}_{0}\right)^{T} \tilde{\Gamma}_{n o i s e}^{-1}\left(v-v_{c}-\tilde{e}_{0}\right)+\beta^{2}\left(v_{c}-\tilde{v}_{0}\right)^{T} \tilde{\Gamma}_{p r_{v}}^{-1}\left(v_{c}-\tilde{v}_{0}\right)\right) .
$$

A equação 3.13 define uma função objetivo sendo possível estimar $v_{c}$ a partir de $v$. Desta forma, esta extensão do método do erro de aproximação pré-condiciona a medida levando em consideração o conhecimento a priori da anatomia de uma população, o erro de aproximação do modelo implícito no D-bar e o erro de medida.

\subsection{Introdução de informação a priori através do pós- processamento das imagens reconstruídas}

Nesta seção é proposta uma forma de melhorar a resolução espacial das imagens reconstruídas através do método D-bar a partir de um conjunto de amostras de uma população e utilizando as propriedades do complemento de Schur para distribuições Gaussianas mostradas na seção 2.5.

A partir de um conjunto de amostras de distribuições de condutividade tridimensionais $\sigma^{3 D}$, equação 3.14, uma estimativa da densidade de probabilidade de uma população $\pi(\sigma)$ pode ser calculada. Para a obtenção de cada uma destas amostras, imagens de tomografia computadorizadas são segmentadas e valores de condutividade atribuídos para cada um dos tecidos discriminados na segmentação.

$$
\left[\sigma^{3 D_{1}}|\ldots| \sigma_{i}^{3 D_{i}}|\ldots| \sigma^{3 D}{ }_{N_{t o t}}\right] \rightarrow \mathcal{N}\left(\bar{\sigma}^{3 D}, \Gamma_{\sigma}^{3 D}\right) \rightarrow \pi(\sigma)
$$


Na equação 3.14 a variável $\sigma^{3 D}{ }_{i}$ representa a $i$-ésima amostra de distribuição de condutividade tridimensional, $N_{\text {tot }}$ o número de amostras utilizadas, $\bar{\sigma}^{3 D}$ a expectância da distribuição de condutividade tridimensional e $\Gamma_{\sigma}^{3 D}$ a sua covariância.

Para cada distribuição de condutividade $\sigma^{3 D}{ }_{i}$ calculam-se duas imagens distintas:

- $\sigma^{2 D}{ }_{i}$, que representa a distribuição de condutividade na seção transversal onde a cinta de eletrodos está posicionada para a $i$-ésima amostra de distribuição de condutividade tridimensional. Tal imagem pode ser obtida através de uma interpolação Gaussiana que leve em consideração apenas uma determinada faixa de altura do domínio 3D.

- $\tilde{\sigma}_{\text {Dbar } i}^{2 D}$, que representa a imagem $\sigma^{2 D}{ }_{i}$ quando reconstruída através do método $D$ bar. Para obter esta imagem, primeiramente calcula-se $V\left(\sigma^{3 D_{i}}\right)$, que é a solução do problema direto da distribuição de condutividade $\sigma^{3 D_{i}}$, e em um segundo momento estima-se através do método $D$-bar a imagem que representa o vetor de tensões calculado.

A Figura 3.1 mostra os passos para a obtenção das imagens propostas. As variáveis $x_{i}$ e $y_{i}$ descritas nesta figura representam as imagens $\sigma^{2 D_{i}}$ e $\tilde{\sigma}_{D b a r}^{2 D}$ vetorizadas. Posteriormente, concatenando os vetores $x_{i}$ e $y_{i}$ obtém-se o vetor $z_{i}$, equação 3.15.

$$
z_{i}=\left[\begin{array}{c}
x_{i} \\
y_{i}
\end{array}\right]
$$

A partir da estimativa do vetor $z_{i}$ de todas as $N_{t o t}$ amostras, estima-se a média $\mu_{z} \mathrm{e}$ a covariância $\Gamma_{z}$ desta distribuição,

$$
\left[z_{1}|\ldots| z_{i}|\ldots| z_{N_{t o t}}\right] \rightarrow \mathcal{N}\left(\mu_{z}, \Gamma_{z}\right)
$$

Avaliando a expectância e a covariância da distribuição do processo z, podemos representar estes parâmetros da seguinte forma,

$$
\mu_{z}=\left[\begin{array}{l}
\mu_{x} \\
\mu_{y}
\end{array}\right] \quad \text { e } \quad \Gamma_{z}=\left[\begin{array}{cc}
\Gamma_{x x} & \Gamma_{x y} \\
\Gamma_{y x} & \Gamma_{y y}
\end{array}\right]
$$

onde $\mu_{x}$ e $\mu_{y}$ são as expectâncias dos processos $x$ e $y, \Gamma_{x x}$ e $\Gamma_{y y}$ as covariâncias de cada um destes processos e $\Gamma_{x y}$ e $\Gamma_{y x}$ as suas covariâncias cruzadas. 


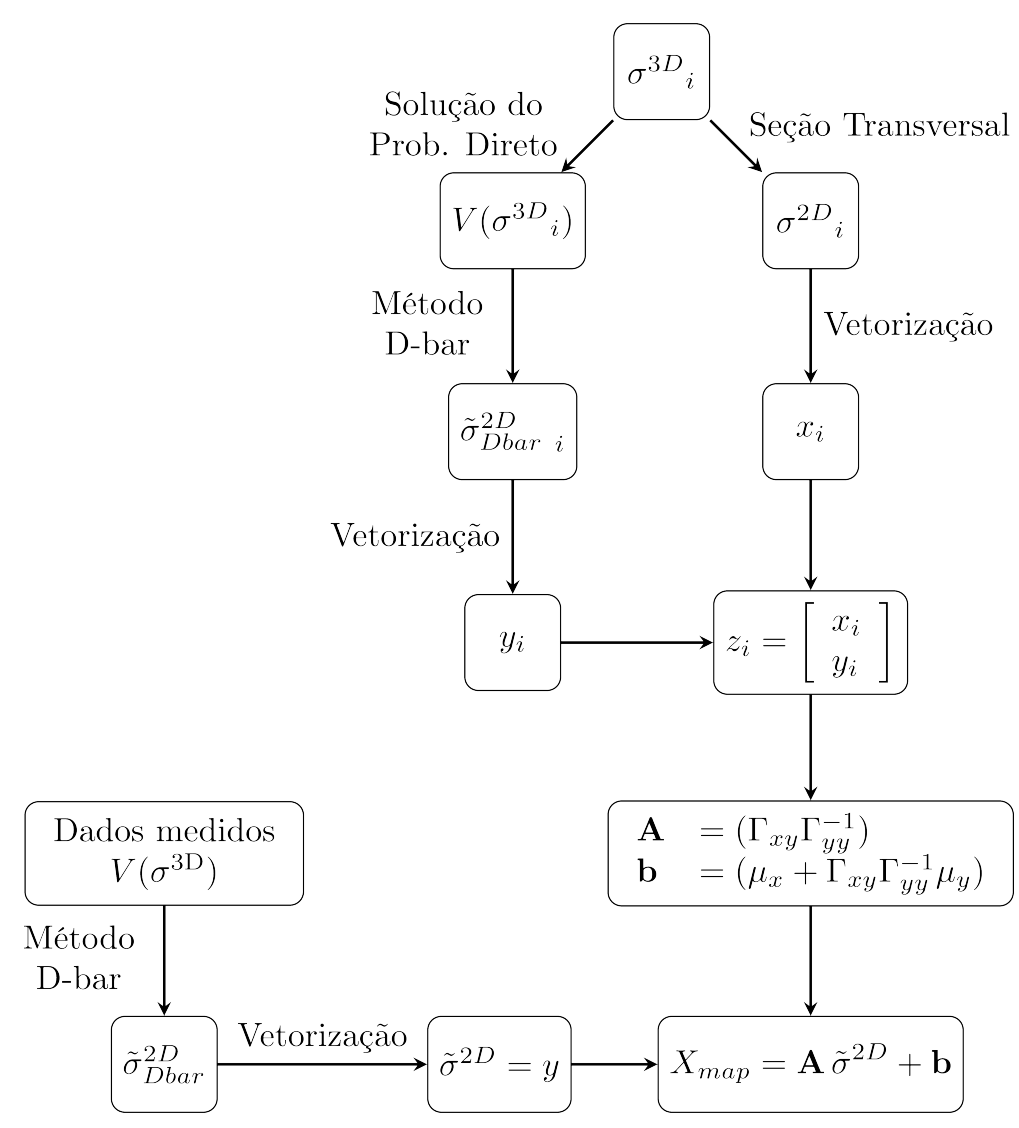

Figura 3.1: Diagrama que descreve o método de introdução de informação a priori através do pós-processamento das imagens reconstruídas.

Como mostrado na Seção 2.5 uma estimativa do vetor $x$ pode ser calculada a partir de $y$ e das estatísticas envolvidas, Equação 2.39. Reordenando a equação, temos um ajuste para as imagens estimadas pelo método D-bar, equação 3.17. Este póscondicionamento da imagem leva em consideração a distribuição de condutividade a priori da população envolvida e a modelagem do problema direto.

$$
\begin{aligned}
X_{\text {map }} & =\mu_{x}+\Gamma_{x y} \Gamma_{y y}^{-1}\left(y-\mu_{y}\right) \\
& =\left(\Gamma_{x y} \Gamma_{y y}^{-1}\right) y+\left(\mu_{x}+\Gamma_{x y} \Gamma_{y y}^{-1} \mu_{y}\right) \\
& =\mathbf{A} y+\mathbf{b}
\end{aligned}
$$




\subsection{Ensaios para avaliação dos métodos}

Para avaliar o desempenho dos métodos propostos nas Seções 3.1 e 3.2, ensaios com fantomas numéricos e experimentais são utilizados. Primeiramente, modela-se o problema direto incluindo o efeito capacitivo dos eletrodos, evitando assim que a capacitância parasita presente nos eletrodos produza artefatos nas imagens. Em seguida, os parâmetros médios do meio, dos eletrodos e da capacitância parasita são calculados para serem utilizados como estimativa inicial no cálculo do erro de aproximação e nas reconstruções. Depois, um conjunto de amostras simuladas de distribuições de condutividades é construído e utilizado para formar expectância e matriz de covariância. Por fim, a partir de fantomas simulados e experimentais, estimam-se as distribuições de condutividade através do método $D$-bar utilizando os métodos propostos e então avaliam-se os resultados obtidos.

\subsubsection{Ensaios em cuba homogênea}

\section{Modelagem do efeito capacitivo}

Umas das características do tomógrafo ACE1 é a presença de capacitâncias parasitas nos eletrodos de medição. Essas capacitâncias deformam o sinal de tensão medido e quando não são levadas em consideração introduzem artefatos significativos na distribuição de condutividade estimada. Para evitar que estes artefatos apareçam na imagem, o efeito capacitivo pode ser modelado através do MEF.

Para a modelagem do efeito capacitivo um elemento concentrado entre cada nó virtual e o terra do sistema pode ser acoplado à malha de elementos finitos, conforme descrito na Seção 2.2.5. Para avaliar o modelo desenvolvido realizam-se medições em cuba com solução salina e comparam-se as tensões medidas pelo tomógrafo com as tensões calculadas pelo problema direto que modela o efeito capacitivo dos eletrodos de medição.

\section{Estimativa inicial e cálculo do erro de aproximação}

Em geral, nos métodos de solução do problema inverso de TIE, uma boa estimativa inicial contribui para que os métodos convirjam com menos iterações (quando iterativos) e apresentem menos artefatos na estimativa final. Para o método de Gauss- 
Newton, por exemplo, a estimativa inicial é importante para o seu bom desempenho, uma vez que este método baseia-se em aproximações lineares da função objetivo.

Através do método de Gauss-Newton abordado na Seção 2.3.1 e utilizando a regularização de Tikhonov generalizado é possível estimar a condutividade média do meio, impedância de contato média e o efeito capacitivo parasita médio. Para avaliar o modelo desenvolvido realizam-se medições em um cuba circular com solução salina de condutividade conhecida e estimam-se os três parâmetros descritos através da minimização do erro quadrático mostrado na equação 2.19. Compara-se o resultado encontrado com o valores esperados da condutividade da solução salina, da impedância de contato e da capacitância equivalente do circuito que compõe o ACE1.

Para incluir o método do erro de aproximação é necessário calcular a expectância $\tilde{e}$ mostrado na equação 2.12. Para isto calculam-se, adotando a mesma estimativa inicial, duas distribuições de tensões, para a primeira utiliza-se um modelo refinado e para a segunda utiliza-se um modelo simplificado. Posteriormente calcula-se a diferença entre elas e adiciona-se o ruído de medição médio, caso ele não seja nulo, estimandose assim a expectância do erro de aproximação $\left(\tilde{e}_{0}\right)$.

Para o cálculo das tensões do modelo refinado é necessária uma estimativa inicial de condutividade. Para encontrar uma estimativa inicial de condutividade, executamse as seguintes etapas para o calculo do erro de aproximação:

\section{Etapas}

1. A partir de um conjunto de medidas de tensão em uma cuba circular preenchida com solução salina, estimar as condições iniciais para o modelo simplificado, sem incorporar o erro de aproximação.

2. Calcular as tensões para o modelo refinado a partir das condições iniciais encontradas.

3. Comparar as tensões medidas na cuba e as tensões calculadas através do modelo refinado e aplicar uma correção da condutividade média do meio para o modelo refinado.

4. Utilizando a nova condutividade média, calcular novamente as tensões para o modelo refinado e para o modelo simplificado utilizando estas condições iniciais corrigidas. 
5. Calcular o erro de aproximação.

6. Estimar as condutividades novamente para o modelo simplificado, com o erro de aproximação não nulo.

\subsubsection{Prior baseado em amostras simuladas}

Uma forma possível de obter amostras para a construção de priors é através de simulações computacionais. Quando conhecida a estatística das características fisiológicas e/ou anatômicas de um certo domínio é possível estimar numericamente distribuições de condutividades e construir priors a partir destas simulações.

Propõe-se a construção de um prior baseado em amostras para ser utilizado em testes de fantoma numéricos e experimentais. A partir do conhecimento da condutividade média e da geometria dos objetos que serão utilizados como targets é possível, por meio de simulações numéricas, estimar um atlas anatômico do fantoma experimental.

\subsubsection{Fantoma numérico}

Fantomas numéricos são usualmente os primeiros testes utilizados para depurar algoritmos de solução do problema inverso de TIE. Estes testes baseiam-se em calcular os potenciais elétricos de uma certa distribuição de condutividade e posteriormente tentar estimá-la por meio do algoritmo que esta sendo depurado. É considerado um crime inverso utilizar o mesmo modelo numérico para calcular as tensões e para realizar a reconstrução da imagem, pois desta forma assume-se que o modelo e a realidade coincidem, o que torna o problema melhor condicionado do que na realidade é.

Uma forma de evitar o crime inverso é utilizar malhas mais refinadas e modelos de eletrodos mais elaborados para resolver o problema direto, adicionar ruído nas tensões calculadas e utilizar parâmetros de entrada para o problema inverso que não coincidam com os utilizados no problema direto.

\subsubsection{Fantoma experimental}

Para a avaliação dos métodos, serão utilizados fantomas experimentais. Através do tomógrafo ACE1 medem-se dados em uma cuba com solução salina de condutividade 
controlada e, em alguns casos, são introduzidos objetos no domínio, com condutividades, formatos e posições conhecidos. Com os dados assim medidos, avalia-se o efeito da introdução de priors no método D-bar. 


\section{Capítulo 4}

\section{Resultados e Discussões}

Neste capítulo são apresentados os resultados para os dois métodos de introdução de informação a priori propostos no Capítulo 3. Seguindo a metodologia proposta, primeiramente é implementado a modelagem do efeito capacitivo. Depois, é mostrado como os parâmetros iniciais do problema direto podem ser estimados. Em seguida, calcula-se a partir desta estimativa inicial o erro de aproximação do modelo que representa o problema direto. Por fim, fantomas numéricos e experimentais são avaliados utilizando os métodos propostos.

\subsection{Modelagem do efeito capacitivo}

O modelo capacitivo mostrado na Seção 2.2.5 foi implementado. A Figura 4.1 mostra a malha utilizada no problema direto simplificado, esta malha é composta por $13 \mathrm{k}$ elementos tetraédricos e está mais refinada próxima aos eletrodos. A partir da malha construída, foi implementado o modelo de eletrodo complete electrode model e um elemento concentrado foi conectado entre cada nó virtual e o terra para modelar o efeito capacitivo nos eletrodos.

Em seguida, uma cuba circular foi preenchida com solução salina $0.18 \mathrm{~S} / \mathrm{m}$ e, utilizando o tomógrafo ACE1, esta cuba foi excitada por meio do padrão de corrente pula-0 e foram medidos o potenciais nos eletrodos. A Figura 4.2 mostra as tensões calculadas (em azul) e medidas (em vermelho) dos 32 eletrodos para o primeiro padrão de injeção de correntes.

Uma vez que a condutividade da solução salina e a impedância de contato não 


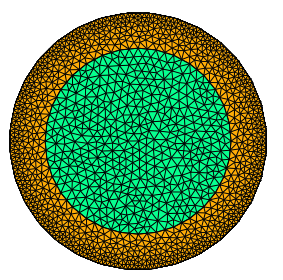

(a) Vista superior

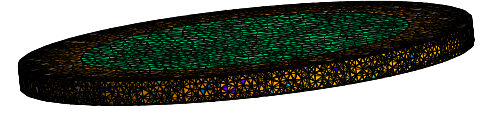

(b) Vista lateral

Figura 4.1: Malha problema direto simplificado, $13 \mathrm{k}$ tetraedros.

apresentam parte imaginária significativa, espera-se que as tensões medidas tenham a parte imaginária desprezível. No entanto, devido às capacitâncias parasitas dos eletrodos, é possível observar que as tensões medidas foram tensões complexas com parte imaginária considerável.

Através da Figura 4.2 é possível observar a influência do efeito capacitivo sobretudo na parte imaginária da tensão medida. Comparando as tensões medidas e as tensões calculadas verifica-se que o modelo capacitivo implementado consegue reproduzir o fenômeno observado nas tensões medidas.

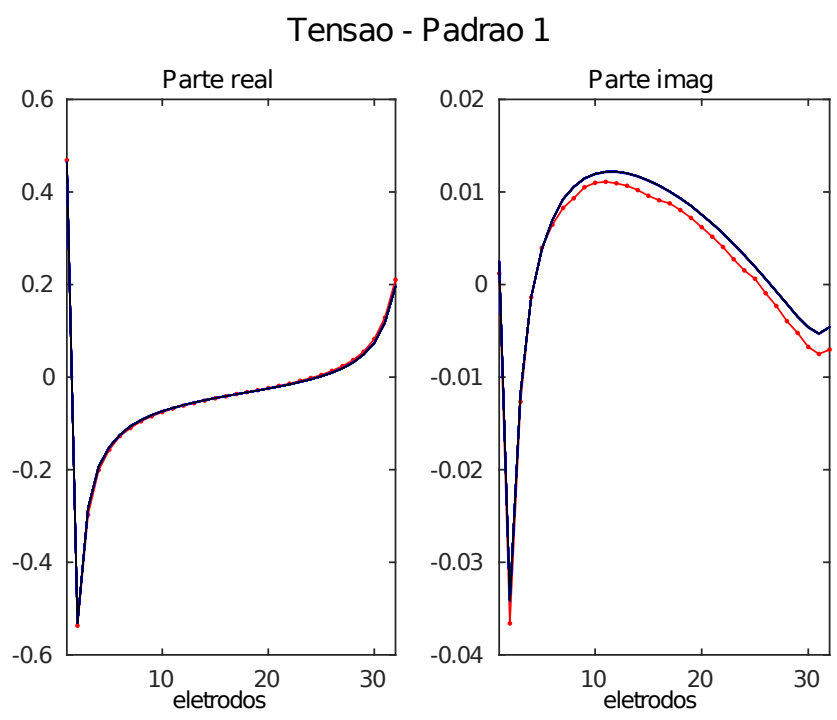

Figura 4.2: Tensão nos eletrodos, Padrão 1.

\subsection{Estimativa inicial e cálculo do erro de aproximação}

Seguindo a metodologia proposta na Seção 2.3.1, utilizou-se uma cuba circular homogênea com solução salina $0.18 \mathrm{~S} / \mathrm{m}$ para estimar os três parâmetros $\left(\sigma_{m e i o}, \sigma_{i m p \_c o n t}\right.$ 
e $Z_{C_{\text {media }}}$ ) utilizados na estimativa inicial do problema e na estimativa do erro de aproximação.

\section{Etapa 1}

A Figura 4.3 mostra o resultado da estimação realizada para a primeira etapa descrita na Seção 3.3.1. Através da Figura 4.3b é possível observar o decrescimento do erro calculado através da Equação 2.19. A capacitância média do sistema (por eletrodo) convergiu para $\approx 80 \mathrm{pF}$, a impedância média de contato ficou em $1.053 \mathrm{~S}$ (para altura $t$ do modelo do eletrodo $10^{-5}$ ) e a condutividade em $0.14645 \mathrm{~S} / \mathrm{m}$. Tanto a impedância de contato quando a condutividade do meio apresentaram parte imaginária.

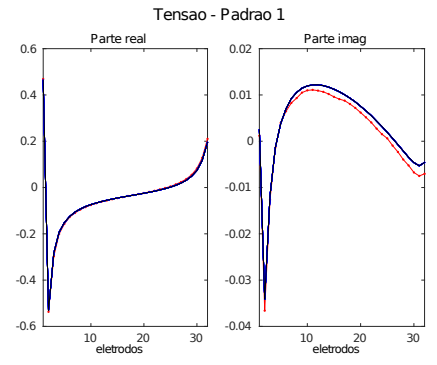

(a) Tensões calculadas (em azul) e medidas (em vermelho), Padrão 1

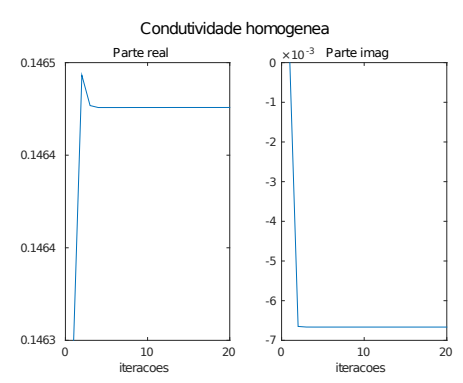

(c) Condutividade homogênea

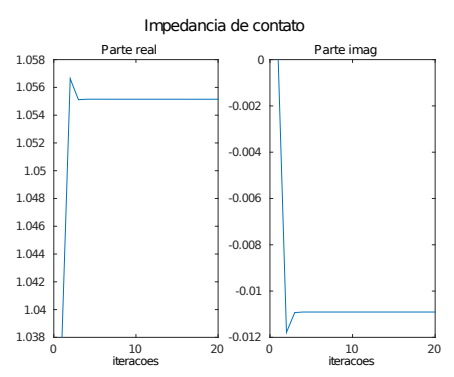

(d) Impedância de Contato

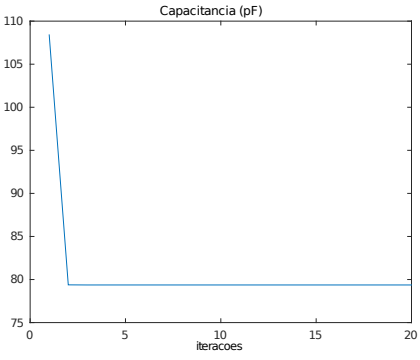

(e) Capacitância

Figura 4.3: Estimativa inicial, modelo simplificado.

\section{Etapas 2 e 3}

A partir das características médias estimadas para o modelo simplificada, foi calculada a distribuição das tensões para o modelo refinado. A Figura 4.5a mostra a comparação entre as tensões medidas e as tensões calculadas através do modelo refinado. Para o modelo refinado foi utilizado os parâmetros médios obtidos na Etapa 1 e uma malha refinada composta por $126 k$ tetraedros (Figura 4.4).

A Figura 4.5a mostra a amplitude das tensões medidas e das tensões calculadas a partir do modelo refinado. Através destas curvas é possível observar que existe uma 


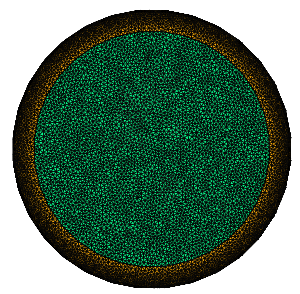

(a) Vista superior

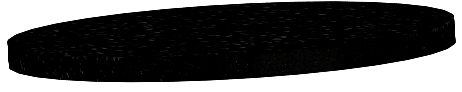

(b) Vista lateral

Figura 4.4: Malha problema direto refinado, $126 k$ tetraedros

superestimação da tensão. Uma vez que a condutividade média é proporcional a tensão calculada, corrigiu-se a condutividade média estimada por meio de um fator multiplicativo proporcional à divisão do pico de tensão medido pelo pico de tensão calculado para o eletrodo de injeção 1. Após a correção da condutividade média, calculou-se novamente o problema direto refinado obtendo a distribuição de tensão mostrada na Figura $4.5 b$, que apresentou tensões mais próximas das tensões medidas.

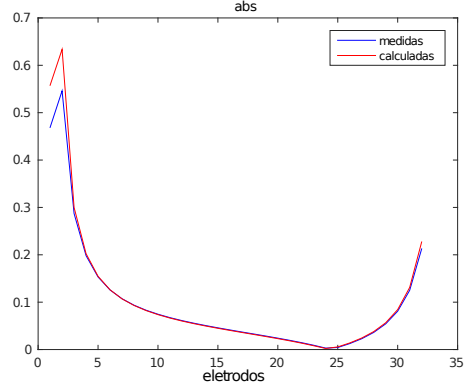

(a) Amplitude das tensões sem correção da cond. média do meio

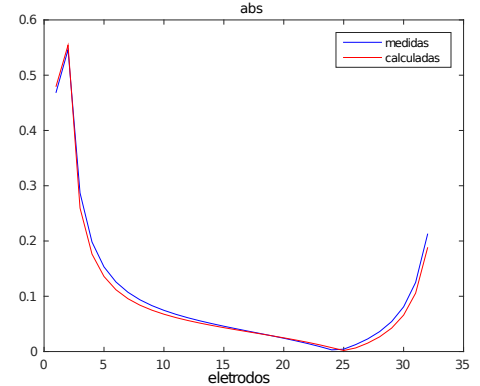

(b) Amplitude das tensões com correção da cond. média do meio

Figura 4.5: Cálculo da tensão nos eletrodos utilizando a malha refinada.

\section{Etapa 4}

Com a estimativa inicial recalculada para o modelo refinado, calculou-se o erro de aproximação $\tilde{e}_{0}$, apresentado na Seção 2.2.3. As Figuras 4.6a e 4.6b mostram, respectivamente, a parte real e a parte imaginária das tensões calculadas utilizando a malha refinada (em vermelho) e utilizando a malha simplificada (em azul). Enquanto as Figuras 4.6c e 4.6d mostram, respectivamente, a parte real e imaginaria do erro de aproximação para o padrão de excitação 1 .

\section{Etapa 5}

A partir dos dados calculados de $\tilde{e}_{0}$, estimam-se novamente os parâmetros iniciais, agora incluindo o erro de aproximação. A Figura 4.7 mostra o resultado desta estima- 


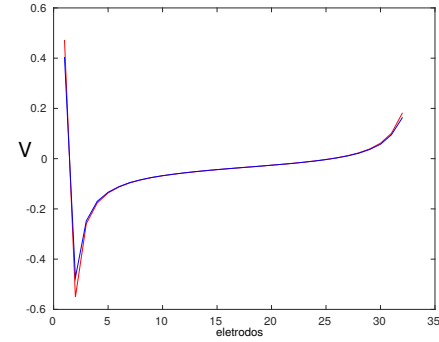

(a) Tensões calculadas, parte real

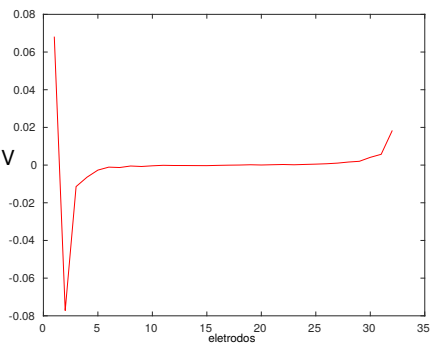

(c) $\tilde{e}_{0}$, parte real.

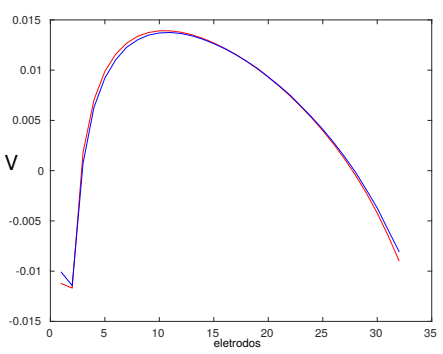

(b) Tensões calculadas, parte imag.

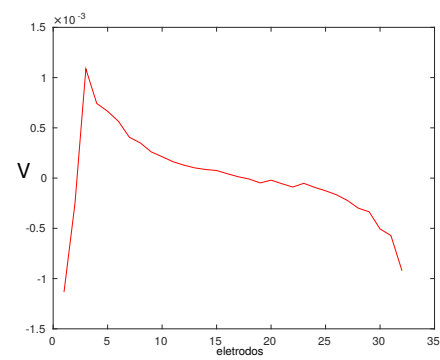

(d) $\tilde{e}_{0}$, parte imag.

Figura 4.6: Cálculo do erro de aproximação $\tilde{e}_{0}$, pula-0 e Padrão 1

ção.

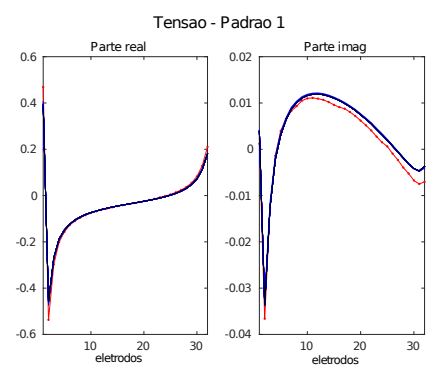

(a) Tensões calculadas (em azul) e medidas (em vermelho), Padrão 1

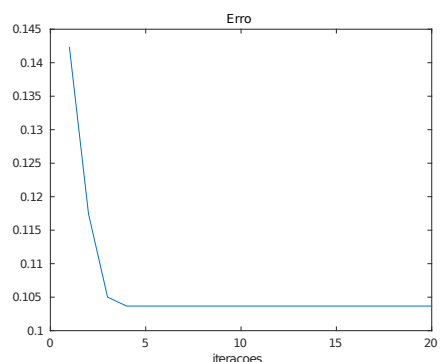

(b) Erro, $r_{2}(\vec{\sigma})$

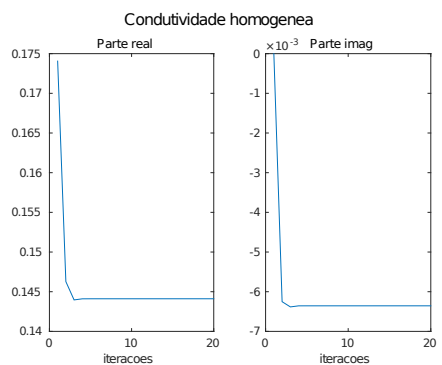

(c) Condutividade homogênea

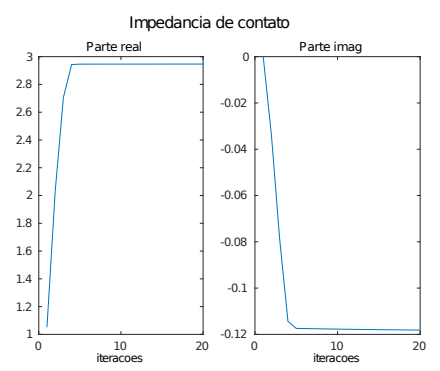

(d) Impedância de Contato

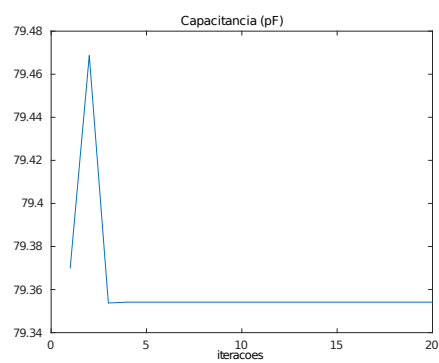

(e) Capacitância

Figura 4.7: Estimativa inicial com o erro de aproximação, malha simplificada.

O resultados mostraram que a capacitância ficou muito próxima da estimada ante- 


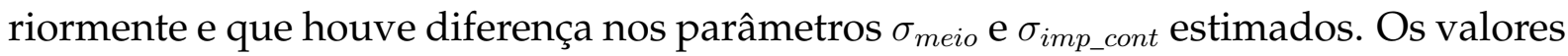
estimados para a condutividade do meio caíram de $0.1464 \mathrm{~S} / \mathrm{m}$ para $0.1441 \mathrm{~S} / \mathrm{m}$, enquanto o valor da impedância de contato (parte real) subiu de $1.055 \mathrm{~S}$ para $2.946 \mathrm{~S}$. Comparando-se as Figuras 4.3b e 4.7b é possível observar que o erro final da função objetivo caiu de $0.1203 \mathrm{~V}^{2}$ para $0.103 \mathrm{~V}^{2}$ quando se utilizou o erro de aproximação.

\subsection{Introdução de informação a priori através do ajuste da tensão}

Para avaliar o método apresentado na seção 3.1, dois conjuntos de fantomas experimentais foram propostos. Utilizou-se o tomógrafo ACE1 para a excitação do domínio e medição das correntes e tensões envolvidas. Como padrão de excitação foi utilizado o pula-0, com amplitude da fonte bipolar em $3.35 \mathrm{~mA}$ e frequência de $125 \mathrm{kHz}$.

No primeiro experimento, Seção 4.3.1, foram medidas as tensões para a cuba circular preenchida apenas com solução salina. Em um segundo momento e utilizando a mesma solução salina, 3 fatias de pepino foram posicionadas em linha. Para uma terceira medição de tensão as mesmas fatias foram reposicionadas na forma de um triângulo.

Em um segundo experimento, Seção 4.3.2, foram medidas as tensões em uma cuba com formato de tórax humano, preenchida apenas com solução salina. Depois, foram introduzidas três peças de ágar com condutividades e formatos conhecidos e realizouse uma nova medição. Posteriormente, retirou-se uma fração de uma das peças de ágar e foram medidas as tensões novamente.

\subsubsection{Fantoma experimental - Cuba circular e pepinos}

\section{Fantomas}

A Figura 4.8 mostra o primeiro fantoma experimental proposto, com 3 fatias de pepinos descascados e posicionados em linha (Figura 4.8a) e em forma triangular (Figura 4.8b). A cuba circular utilizada tem raio $0.15 \mathrm{~m}$, com 32 eletrodos equidistantes de $25 \mathrm{~mm}$ de largura. Para este experimento a cuba de acrílico foi preenchida com solução salina de condutividade $0.18 \mathrm{~S} / \mathrm{m}$, verificada através de um condutivímetro modelo 


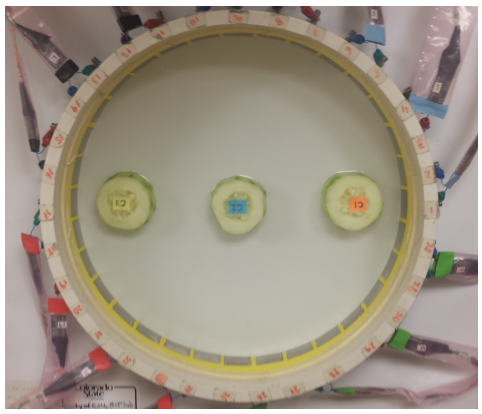

(a) Em linha

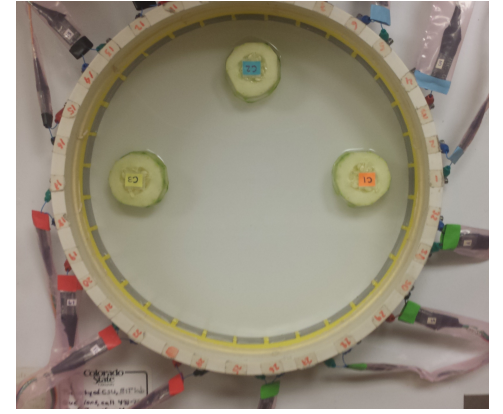

(b) Em posição triangular

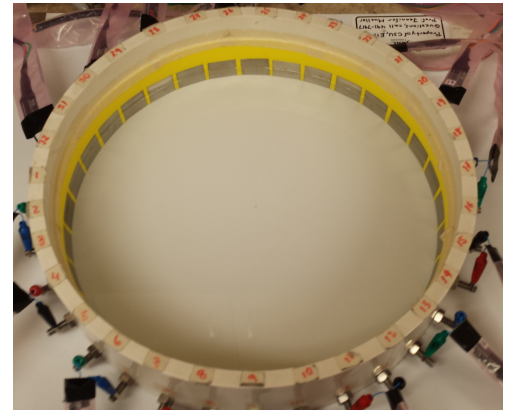

(c) Meio homogêneo

Figura 4.8: Fantoma experimental, cuba circular com 3 pepinos.

\section{Prior baseado em amostras simuladas}

Utilizando a malha mostrada na Figura 4.1, foram sorteadas $200 \mathrm{k}$ amostras de distribuições de condutividade simulando o fantoma experimental proposto. Para cada amostra, sorteou-se posição, raio e condutividade de um objeto cilíndrico em uma cuba homogênea, Figura 4.9. A condutividade da solução salina e do objeto foram sorteadas seguindo distribuições Gaussianas, enquanto o raio do objeto e a sua posição seguiram distribuições uniformes.
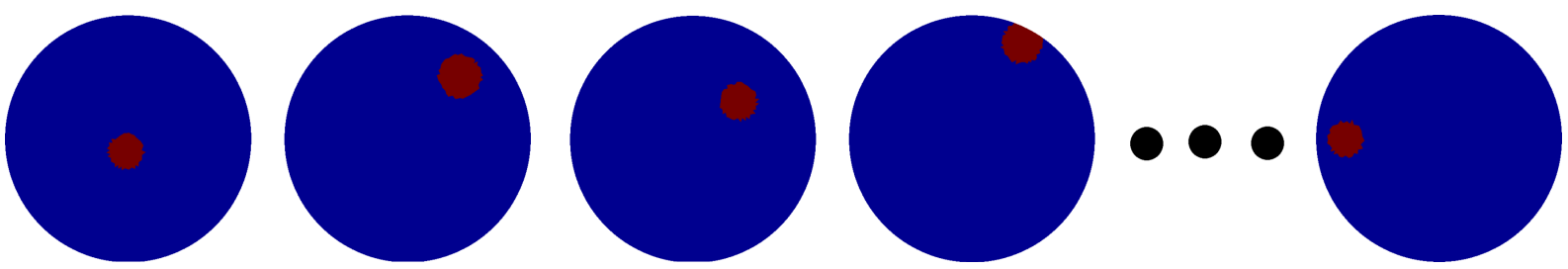

Figura 4.9: Amostras simuladas, fatias de pepino.

Utilizando o problema direto simplificado, calculou-se um vetor de tensões para cada uma das amostras geradas. Posteriormente, o vetor de tensões médio $\tilde{v}_{0}$ e a matriz de covariância $\tilde{\Gamma}_{p r_{v}}$ foram calculadas para representar este conjunto de amostras.

\section{Imagens reconstruídas}

Para cada uma das tensões medidas do fantoma proposto, foi estimado, através da minimização da Equação 3.13, vetores de tensões para diversos valores de $\beta$. Para a minimização da função objetivo foi utilizada o algoritmo para solução de problemas não lineares fminsearch da plataforma MATLAB ${ }^{\circledR}$. 
Através do algoritmo $D$-bar, descrito na Seção 2.3.2, foram estimados dois conjuntos de dados, para o Caso 1 foram minimizadas as tensões utilizando como erro de modelo $\tilde{e}_{0}$ (equação 3.13) o erro de aproximação calculado para a cuba circular. Em um segundo momento, Caso 2, o erro de aproximação $\tilde{e}_{0}$ foi substituído pela diferença entre a tensão basal medida na cuba homogênea e a tensão basal calculada.

\section{Caso 1}

A Figura 4.10 mostra as imagens estimadas a partir das tensões medidas do fantoma em que as fatias de pepino estão em linha, Figura 4.8a. A Figura 4.10a mostra a estimação para a tensão sem ajuste, enquanto nas outras imagens é mostrado as estimações para diversos valores do parâmetro de regularização $\beta$.

Através das imagens é possível perceber que o melhor resultado para o experimento em que as fatias de pepino estavam em linha ocorreu para $\beta^{2}=10^{-4}$, onde é possível diferenciar os 3 objetos. Quanto menor o valor do parâmetro de regularização $\beta$ mais próxima a imagem ficou da imagem estimada sem o ajuste das tensões. Apesar das imagens apresentarem muitos artefatos e baixa resolução, houve uma melhora significativa quando o vetor de tensões foi corrigido.

A Figura 4.11 mostra imagens estimadas, para diferentes valores de $\beta$, a partir das tensões medidas do fantoma em que as fatias de pepino foram posicionadas em forma de triângulo, Figura 4.8b. A Figura 4.11a mostra a estimação para a tensão sem ajuste, enquanto nas outras imagens é mostrado as estimações para diversos valores do parâmetro de regularização $\beta$.

Para o experimento com as fatias de pepino posicionadas em forma triangular o melhor resultado ocorreu para $\beta^{2}=10^{-5}$, onde os três objetos apresentaram visualmente formato mais circular e menor erro de posição. Quando $\beta^{2}=10^{-4}$, melhor caso para o fantoma com as fatias de pepino em linha, os objetos foram deformados e deslocados para a borda do domínio.

\section{Caso 2}




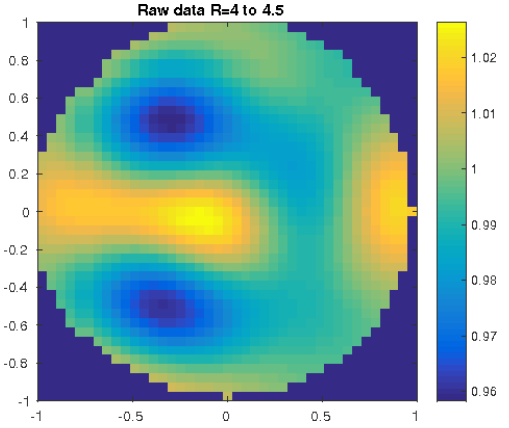

(a) Tensões inalteradas

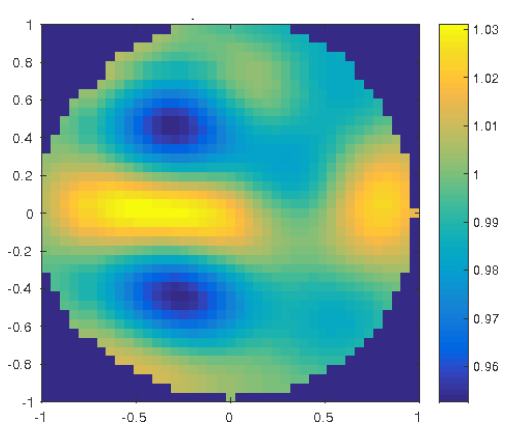

(d) $\beta^{2}=10^{-5}$

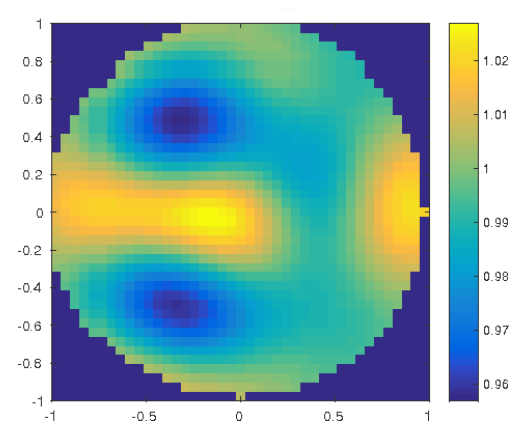

(b) $\beta^{2}=10^{-7}$

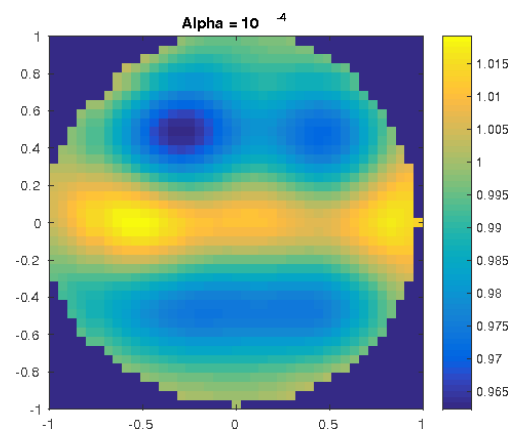

(e) $\beta^{2}=10^{-4}$

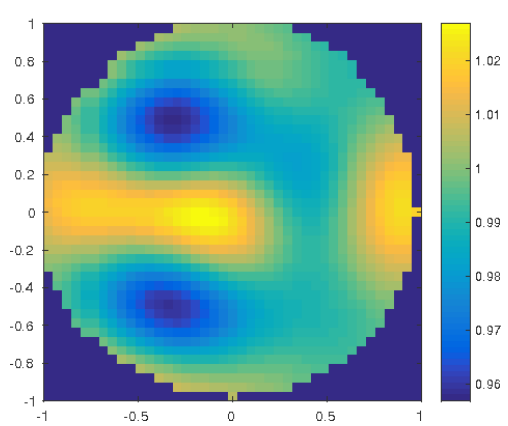

(c) $\beta^{2}=10^{-6}$

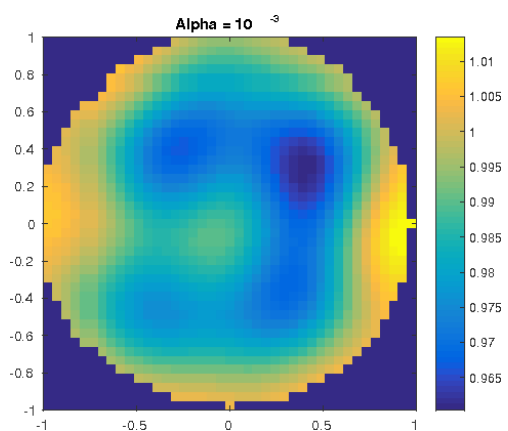

(f) $\beta^{2}=10^{-3}$

Figura 4.10: Caso 1, fantoma com pepinos posicionados em linha.

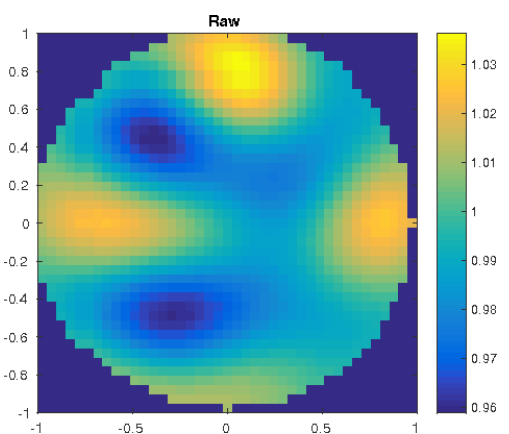

(a) Tensões inalteradas

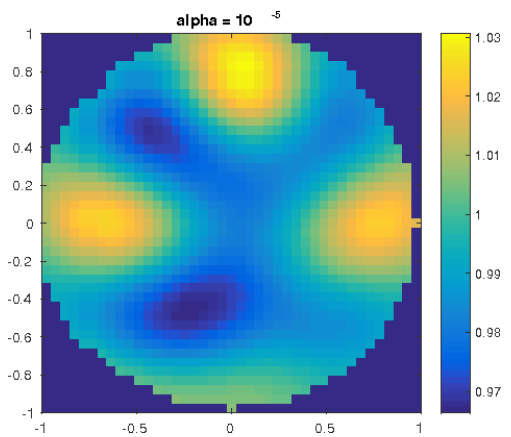

(d) $\beta^{2}=10^{-5}$

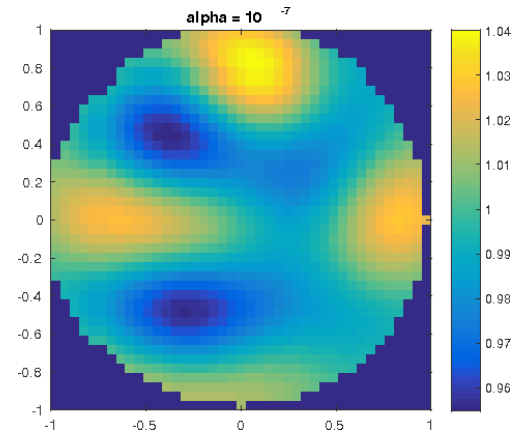

(b) $\beta^{2}=10^{-7}$

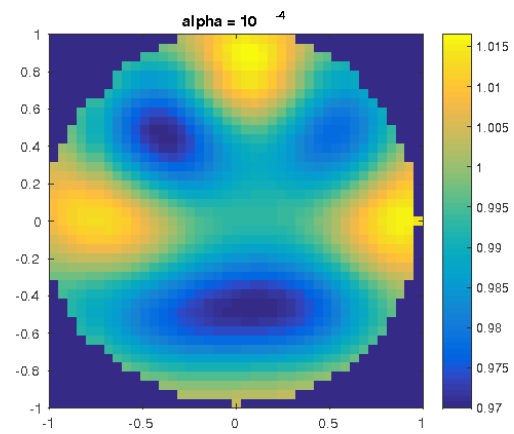

(e) $\beta^{2}=10^{-4}$

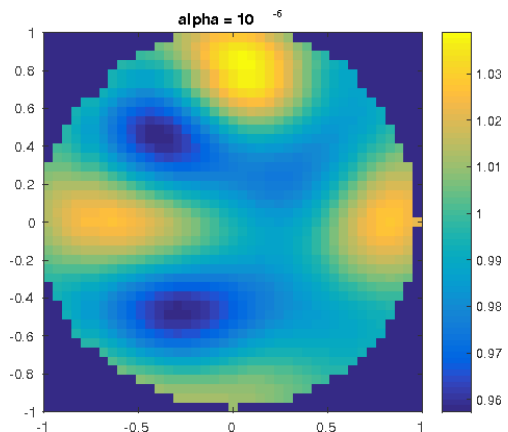

(c) $\beta^{2}=10^{-6}$

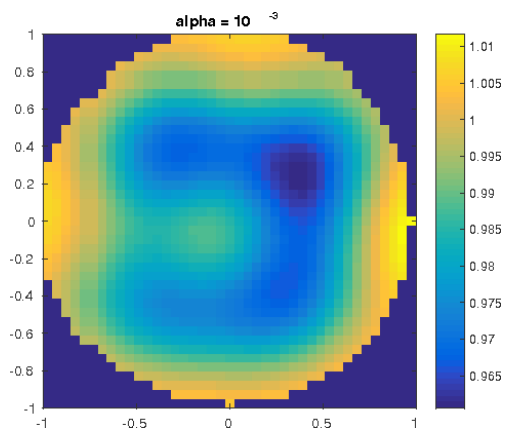

(f) $\beta^{2}=10^{-3}$

Figura 4.11: Caso 1, fantoma com pepinos posicionados em forma de triângulo. 
Para a estimação das imagens no Caso 2 o erro $\tilde{e}_{0}$ foi substituído por,

$$
\tilde{e}_{0}^{*}=v_{\text {med_basal }}-v_{\text {cal_basal }},
$$

onde $v_{\text {med_basal }}$ é a tensão medida na cuba homogênea e $v_{\text {cal_basal }}$ a tensão calculada através do modelo simplificado. Desta forma o erro de aproximação é calculado levando em consideração as tensões medidas para a cuba homogênea.

A Figura 4.12 mostra as imagens estimadas para diversos valores de $\beta$ a partir das tensões medidas do fantoma em que as fatias de pepino estão em linha. Nesta estimação o melhor resultado ocorreu para $\beta^{2}=10^{-5}$, onde é possível observar os três objetos com formato e posições esperados. Vale observar que, mesmo no melhor caso, a condutividade do objeto central está subestimada em relação aos outros dois objetos.

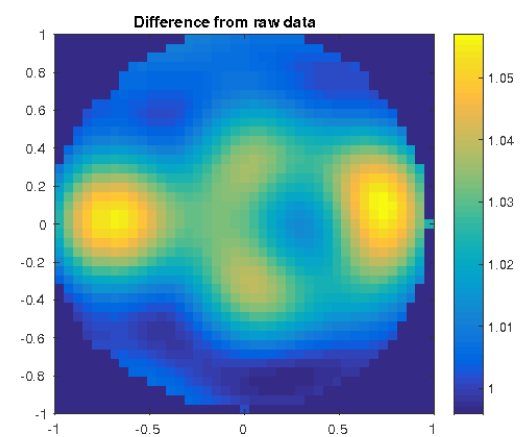

(a) Tensões inalteradas

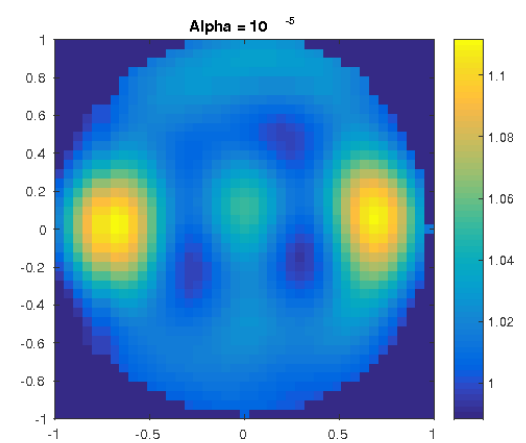

(d) $\beta^{2}=10^{-5}$

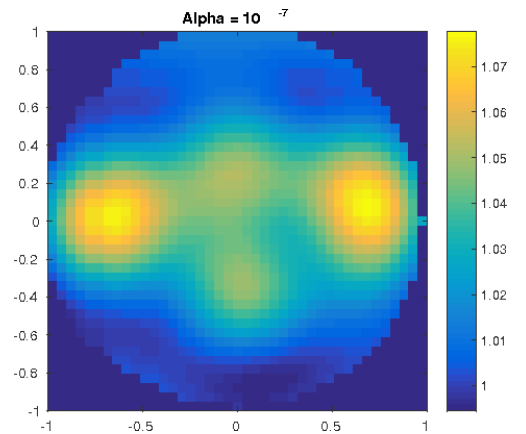

(b) $\beta^{2}=10^{-7}$

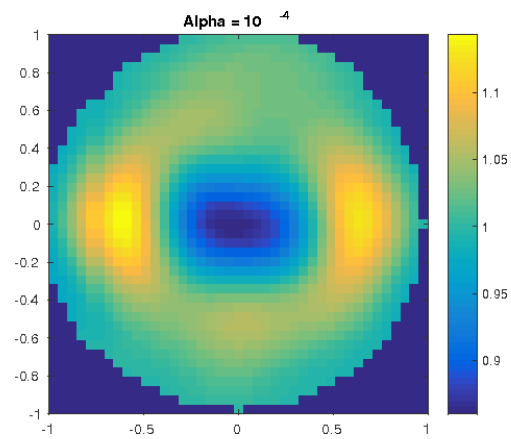

(e) $\beta^{2}=10^{-4}$

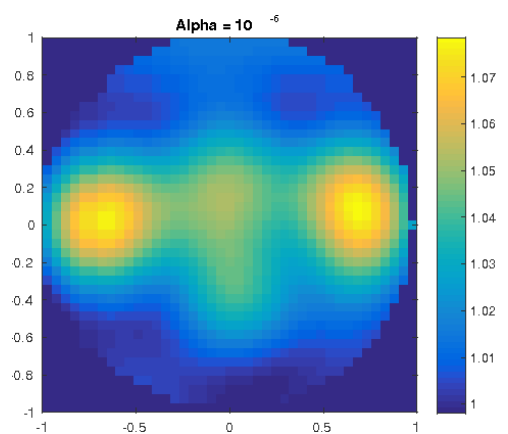

(c) $\beta^{2}=10^{-6}$

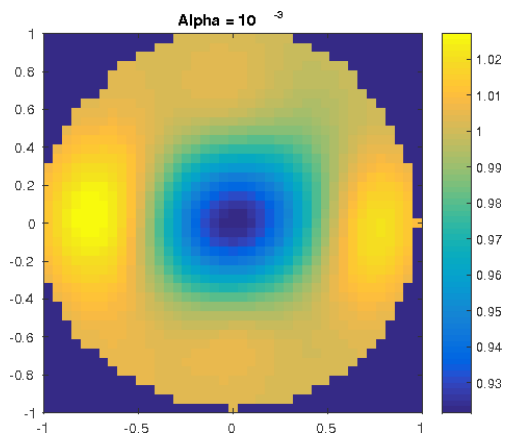

(f) $\beta^{2}=10^{-3}$

Figura 4.12: Caso 2, fantoma com pepinos posicionados em linha.

A Figura 4.13 mostra os resultados encontrados para o caso onde as fatias foram posicionadas em forma de triângulo. Comparando-se as imagens, é possível perceber que com o aumento do parâmetro $\beta$ os objetos apresentam uma forma circular melhor definida, no entanto ocorre o aparecimento de um artefato no centro da imagem. 


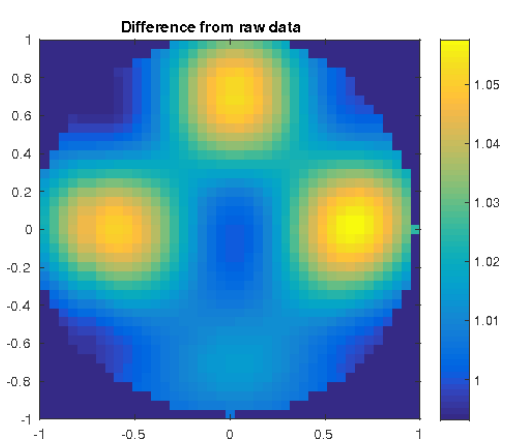

(a) Tensões inalteradas

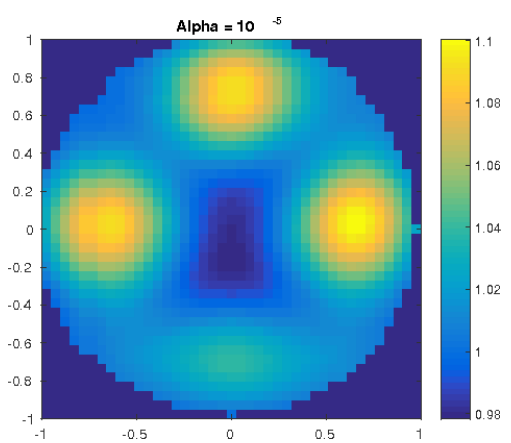

(d) $\beta^{2}=10^{-5}$

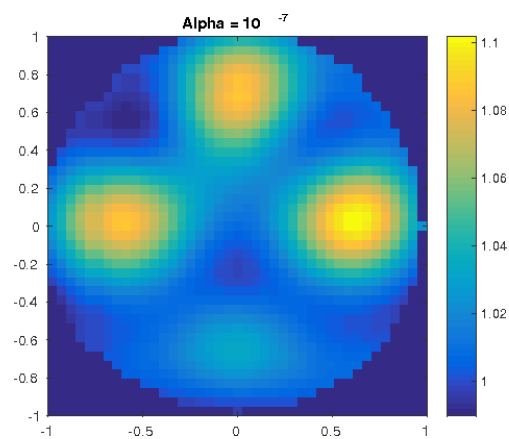

(b) $\beta^{2}=10^{-7}$

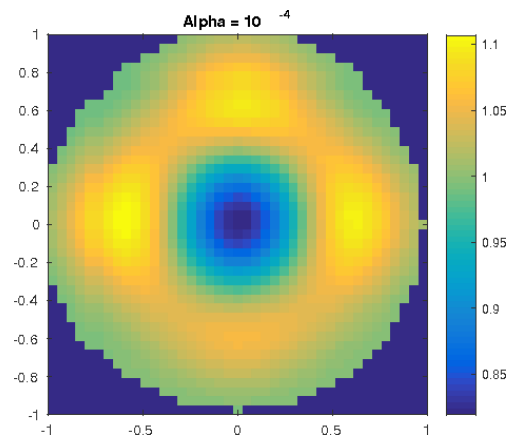

(e) $\beta^{2}=10^{-4}$

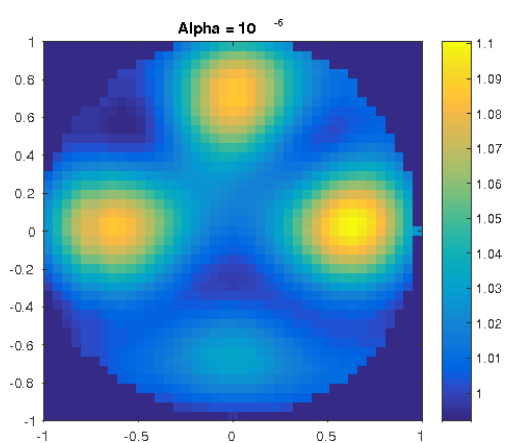

(c) $\beta^{2}=10^{-6}$

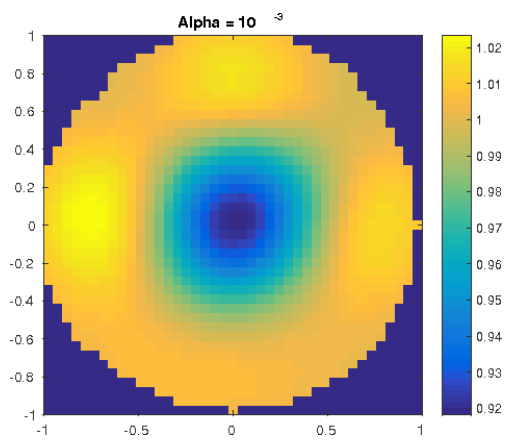

(f) $\beta^{2}=10^{-3}$

Figura 4.13: Caso 2, fantoma com pepinos posicionados em forma de triângulo.

\subsubsection{Fantoma experimental - Cuba em formato de tórax humano e objetos feitos de ágar}

\section{Fantomas}

A Figura 4.14 mostra o segundo fantoma experimental proposto para avaliar a introdução de informação a priori através do ajuste da tensão. Foram preparados 3 objetos a base de ágar para simular características fisiológicas e anatômicas do coração e dos pulmões de um indivíduo. Ajustou-se a condutividade dessas peças para condutividades próximas das esperadas para coração e pulmão de pacientes. A cuba foi preenchida com solução salina e os objetos foram cortados e posicionados no centro da cuba, simulando a anatomia e posição desses órgãos.

A cuba de acrílico tem o formato de um tórax humano e apresenta 32 eletrodos (25 mm de largura) no seu entorno. Para este experimento foi utilizada uma solução salina de condutividade $0.20 \mathrm{~S} / \mathrm{m}$, uma a peça de ágar com condutividade $0.42 \mathrm{~S} / \mathrm{m}$ representando o coração e mais duas peças de ágar com condutividades $0.08 \mathrm{~S} / \mathrm{m}$ representando os pulmões. A medição da propriedade elétrica de cada um dos órgãos e da solução salina foi realizada através de um condutivímetro modelo Omega CDH221. 
Primeiramente realizou-se a medição das tensões na cuba em formato de tórax humano preenchida apenas com solução salina. Em um segundo momento, os objetos feitos de ágar foram inseridos na cuba e novas medições foram realizadas. Por fim, foi retirada a parte superior da peça de ágar que representava o pulmão direito do paciente. Para as medições, utilizou-se o tomógrafo ACE1 ajustado para excitar o domínio através do padrão de corrente pula-0.

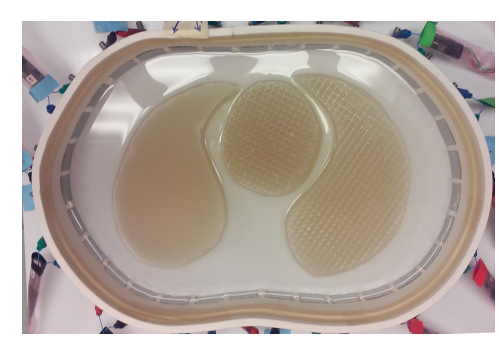

(a) Coração e pulmões intactos

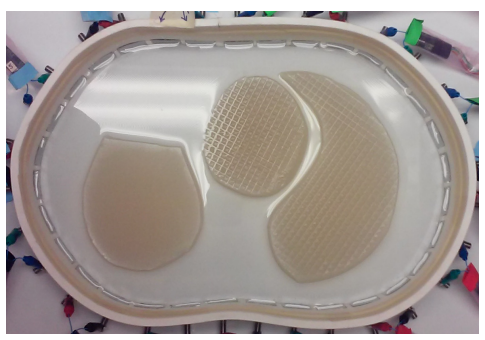

(b) Pulmão direito de ágar cortado

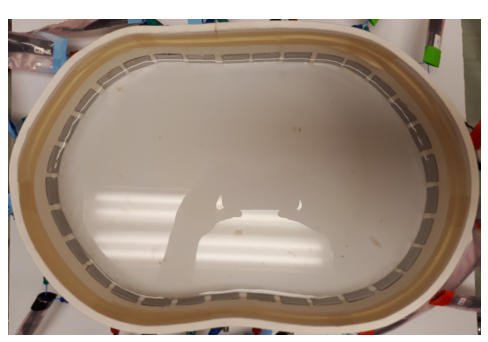

(c) Meio homogêneo

Figura 4.14: Fantoma experimental, cuba em formato de tórax humano com órgãos feitos de ágar.

\section{Prior baseado em amostras simuladas}

Para que o método proposto na seção 3.1 seja avaliado por meio de fantomas experimentais, foi desenvolvido um prior baseado em amostras simuladas. A partir da imagem do fantoma experimental da cuba em formato de tórax humano com os órgãos intactos, Figura 4.14a, foi descrito o formato da cuba, a posição dos eletrodos e o contorno dos órgãos envolvidos. A Figura 4.15 exemplifica o processo descrito, onde foram delimitadas as regiões do coração (verde), pulmão esquerdo (rosa) e pulmão direito (azul).

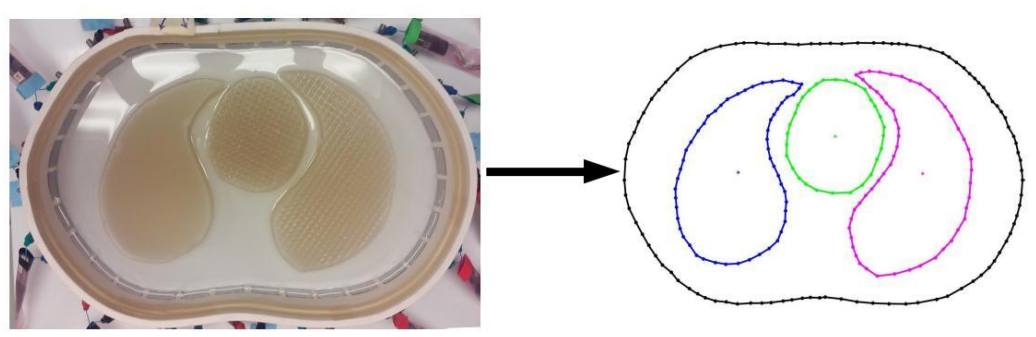

Figura 4.15: Identificação dos contornos dos órgãos e da cuba.

Utilizando os pontos que descrevem o contorno da cuba e a posição dos eletrodos, foi construída uma malha de elementos finitos representando a cuba em formato de 
tórax humano, Figura 4.16. Esta malha é composta por $13 \mathrm{k}$ elementos tetraédricos, tem a mesma altura da coluna de solução salina do experimento proposto e apresenta maior refinamento próximo à região dos eletrodos.

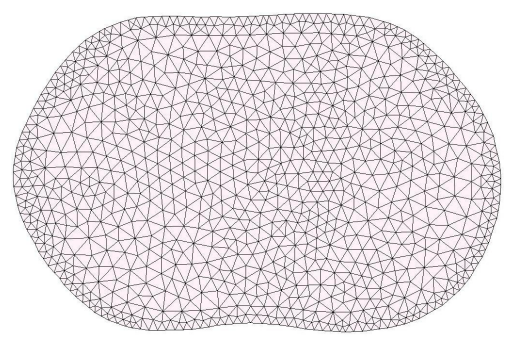

(a) Vista superior

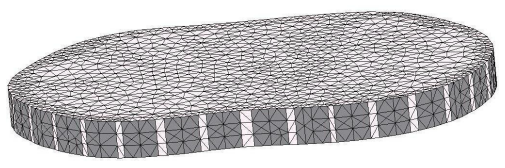

(b) Vista lateral

Figura 4.16: Malha de elementos finitos em formato de tórax humano, 13 k elementos

A partir do contorno das regiões (coração, pulmão direito e pulmão esquerdo) e conhecidas as coordenadas dos centroides de cada um dos elementos tetraédricos da malha é possível determinar quais são os centroides que se encontram em cada uma das regiões. A Figura 4.17 exemplifica este processo, onde são identificados os elementos da malha pertencentes a cada uma das regiões.

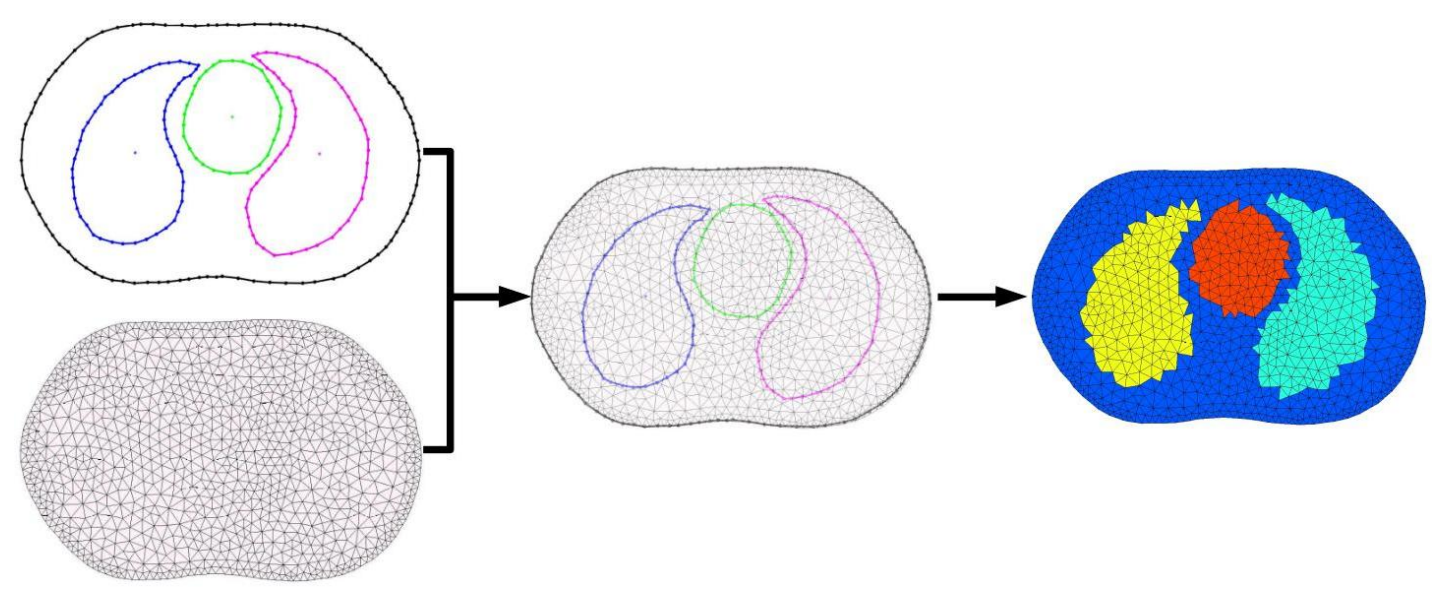

Figura 4.17: Processo de identificação das regiões na malha de elementos finitos.

Para a construção do prior baseado em amostras simuladas foram simulados $15 \mathrm{k}$ amostras. Utilizando como referência a malha de elementos finitos mostrada na Figura 4.16 e os contornos das regiões dos pulmões e coração identificados na Figura 4.15, foi 
sorteado, por amostra, os seguintes parâmetros para cada região,

$$
\text { Região } \Rightarrow \begin{cases}\text { Condutividade }\left(\sigma_{\text {região }}\right) & \sim N\left(\sigma_{\text {região }}, s t d_{\text {região }}\right) \\ \text { Dilatação }(A) & =U\left(A_{\text {min }}, A_{\text {max }}\right) \\ \text { Deslocamento horizontal }\left(x_{\text {shift }}\right) & =U\left(x_{\text {min }}, x_{\text {max }}\right) \\ \text { Deslocamento vertical }\left(y_{\text {shift }}\right) & =U\left(y_{\min }, y_{\max }\right) \\ \text { Rotação }\left(\theta_{\text {rot }}\right) & =U\left(\theta_{\min }, \theta_{\max }\right)\end{cases}
$$

onde a condutividade de cada região foi sorteada seguindo distribuições Gaussianas e os demais parâmetros $A, x_{\text {shift }}, y_{\text {shift }}$ e $\theta_{\text {rot }}$ foram sorteados segundo distribuições uniformes. Além disto, cada amostra sorteada contém uma pequena deformação em um dos pulmões, representando uma imperfeição, alteração ou patologia. Estas pertubações são simuladas a partir da subtração de um objeto circular de tamanho e posição aleatórios no pulmão selecionado.

Conforme o conjunto de parâmetros sorteados para cada região, os contornos foram escalados, deslocados e rotacionados. Em seguida, esses contornos, agora modificados, foram identificados na malha de elementos finitos e então foi atribuído o valor da condutividade sorteada de cada região. A Figura 4.18 mostra como o processo foi realizado, onde $\tilde{\sigma}_{1}$ é o vetor de condutividades que descreve a amostra sorteada 1 na malha de elementos finitos, $\tilde{\sigma}_{N}$ o vetor de condutividades que descreve a enésima amostra sorteada na malha de elementos finitos e $N_{\text {amostras }}$ o número total de amostras simuladas.

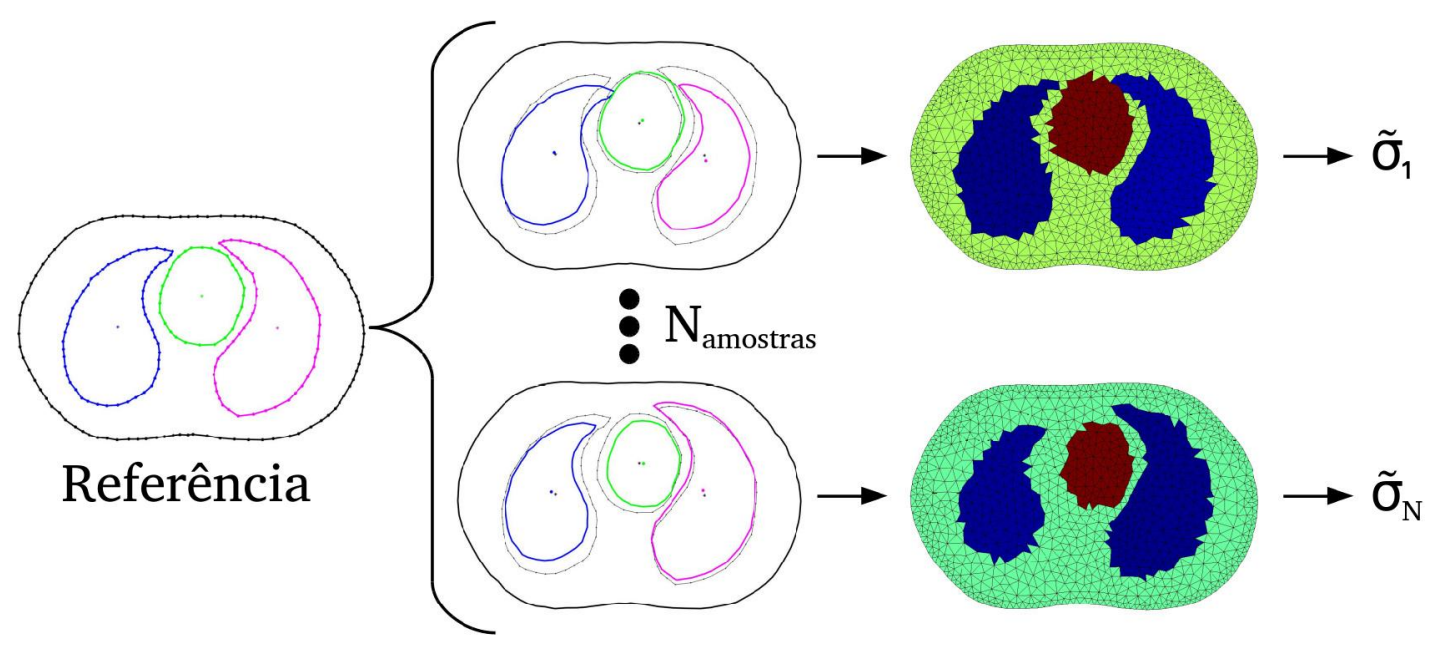

Figura 4.18: Simulação das amostras do prior.

Utilizando o problema direto simplificado, calculou-se um vetor de tensões para 
cada uma das amostras geradas. Posteriormente, o vetor de tensões médio $\tilde{v}_{0}$ e a matriz de covariância $\tilde{\Gamma}_{p r_{v}}$ foram calculadas para representar este conjunto de amostras.

\section{Imagens reconstruídas}

Para cada uma das tensões medidas do fantoma proposto, foi estimado, através da minimização da Equação 3.13, vetores de tensões para diversos valores de $\beta$. Para a minimização da função objetivo foi utilizada o algoritmo para solução de problemas não lineares fminsearch da plataforma MATLAB ${ }^{\circledR}$.

Através do algoritmo $D$-bar, descrito na Seção 2.3.2, foram estimados dois conjuntos de dados, para o Caso 1 foram minimizadas as tensões utilizando como erro de modelo $\tilde{e}_{0}$ (equação 3.13) o erro de aproximação calculado para a cuba circular. Em um segundo momento, Caso 2, o erro de aproximação $\tilde{e}_{0}$ foi substituído pela diferença entre a tensão basal medida na cuba homogênea e a tensão basal calculada.

\section{Caso 1}

A Figura 4.19 mostra as imagens estimadas a partir das tensões medidas do fantoma experimental na cuba em formato de tórax humano com os órgãos feitos de ágar intactos. A Figura 4.19a mostra a estimação para a tensão sem ajuste, enquanto nas outras imagens são mostradas as estimações para diversos valores do parâmetro de regularização $\beta$.

Através das imagens é possível perceber que o resultado que apresentou a melhor resolução espacial para o experimento onde os órgãos estão intactos ocorreu para $\beta^{2}=10^{-4}$. Para este valor de $\beta$ a posição e o formato do coração e dos pulmões estão mais próximos do formato real das peças de ágar. Quanto menor o valor do parâmetro de regularização mais próxima a imagem ficou da imagem estimada sem o ajuste das tensões. Por meio deste fantoma é possível verificar que houve uma melhora significativa quando o vetor de tensões foi corrigido.

A Figura 4.20 mostra as imagens estimadas a partir das tensões medidas do fantoma experimental para o caso onde a parte superior do pulmão de ágar direito foi cortada, Figura 4.14b. A Figura 4.20a mostra a estimação para a tensão sem ajuste, enquanto nas outras imagens é mostrado as estimações para diversos valores do parâ- 


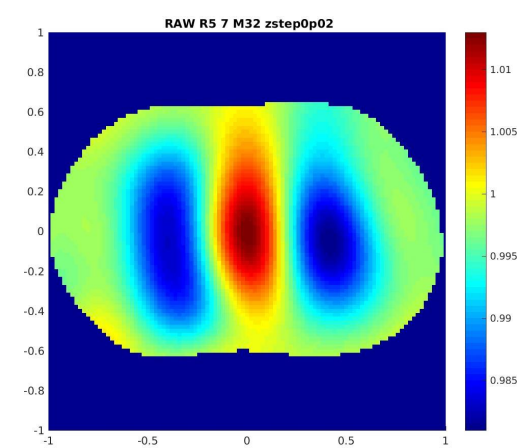

(a) Tensões inalteradas

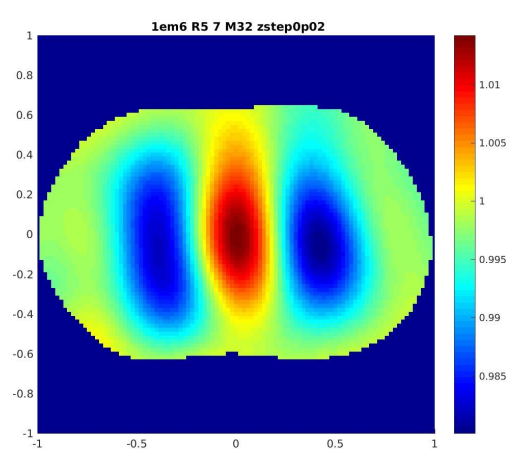

(b) $\beta^{2}=10^{-6}$

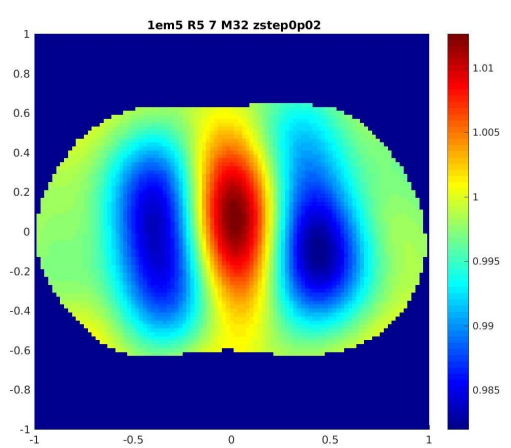

(c) $\beta^{2}=10^{-5}$

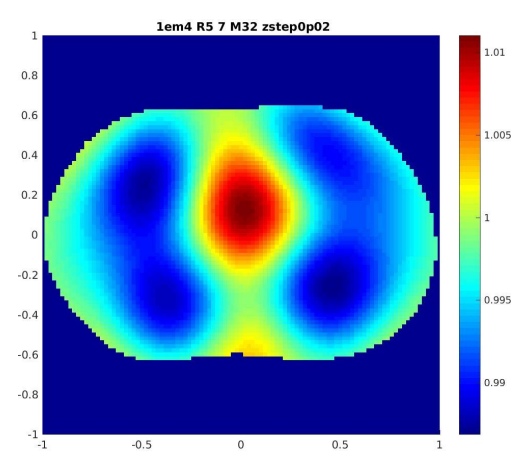

(d) $\beta^{2}=10^{-4}$

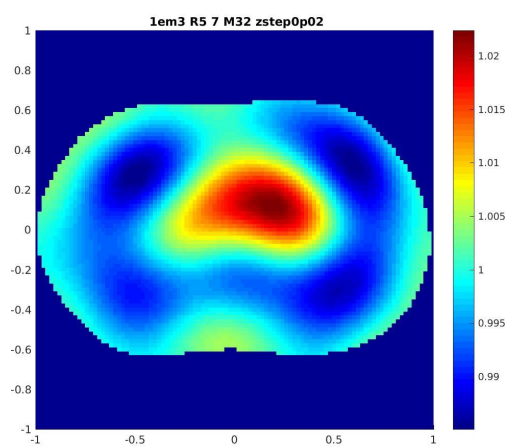

(e) $\beta^{2}=10^{-3}$

Figura 4.19: Caso 1, fantoma com cuba em formato de tórax humano com órgãos de ágar intactos.

metro de regularização $\beta$.

Através das imagens é possível perceber que o resultado que apresentou a melhor resolução espacial para o experimento onde uma parte do pulmão de ágar direito foi retirada ocorreu para $\beta^{2}=10^{-4}$, seguindo o caso onde os órgãos estão intactos. Para este valor de $\beta$ a posição e o formato do coração e dos pulmões estão mais próximos do formato real das peças de ágar. Quanto menor o valor do parâmetro de regularização mais próxima a imagem ficou da imagem estimada sem o ajuste das tensões.

\section{Caso 2}

Para a estimação das imagens no Caso 2 o erro $\tilde{e}_{0}$ foi substituído por,

$$
\tilde{e}_{0}^{*}=v_{\text {med_basal }}-v_{\text {cal_basal }},
$$

onde $v_{\text {med_basal }}$ é a tensão medida na cuba homogênea e $v_{\text {cal_basal }}$ a tensão calculada através do modelo simplificado. Desta forma o erro de aproximação é calculado levando 


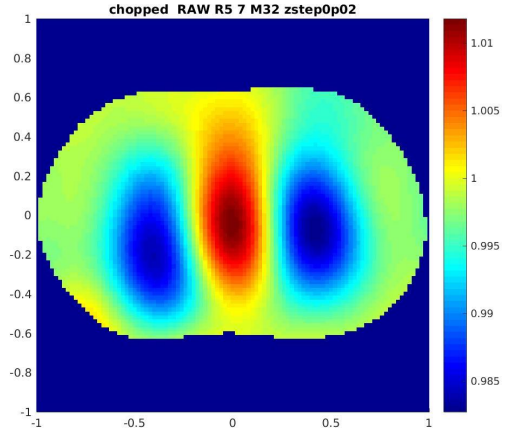

(a) Tensões inalteradas

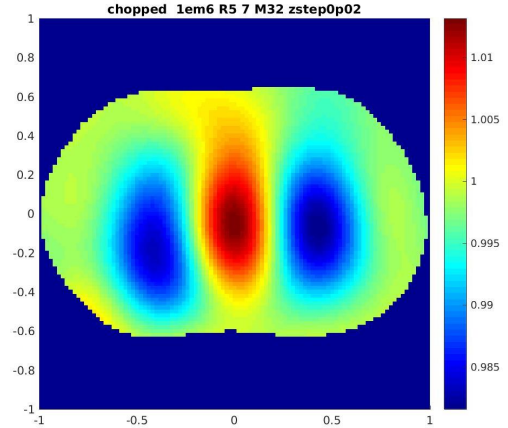

(b) $\beta^{2}=10^{-6}$

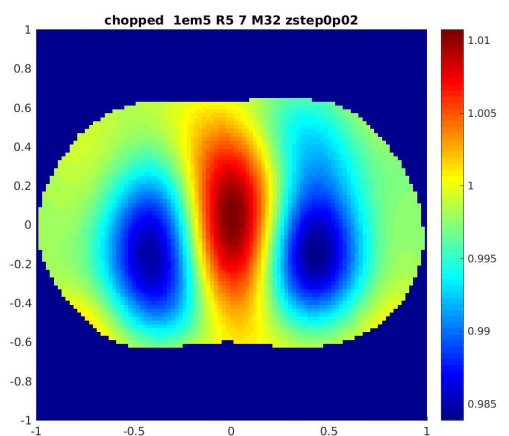

(c) $\beta^{2}=10^{-5}$

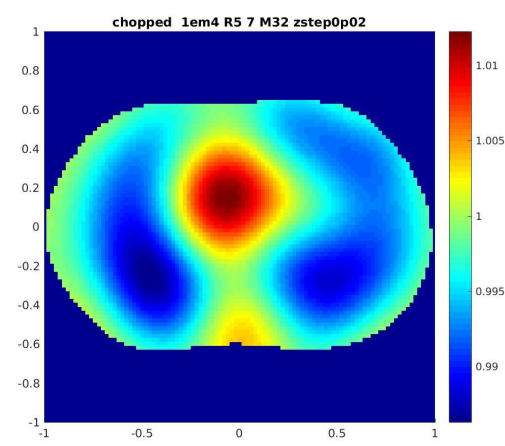

(d) $\beta^{2}=10^{-4}$

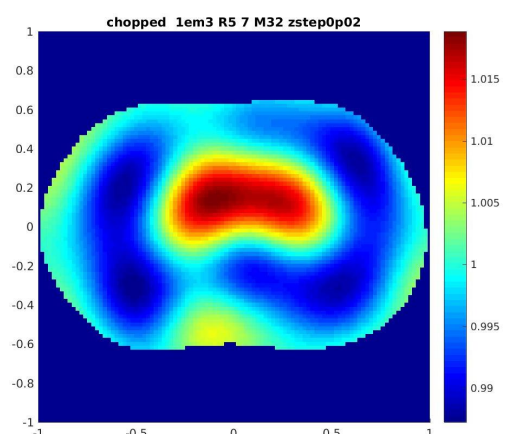

(e) $\beta^{2}=10^{-3}$

Figura 4.20: Caso 1, fantoma com cuba em formato de tórax humano com pulmão de ágar direito cortado.

em consideração as tensões medidas para a cuba homogênea.

A Figura 4.21 mostra as imagens estimadas para o caso onde os pulmões de ágar estavam intactos. A partir das imagens é possível observar que a reconstrução com o formato e posição mais próximos do fantoma ocorreu para $\beta^{2}=10^{-4}$. Na Figura $4.21 \mathrm{~d}$ é possível observar que a posição do coração está mais próxima do experimental, no entanto o seu contorno continuou deformado.

A Figura 4.22 mostra os resultados encontrados o experimento onde parte do pulmão de ágar direito foi retirada. A partir das imagens é possível observar que o resultado que reconstruiu a distribuição de condutividade com o formato e posição mais próximos do real ocorreu para $\beta^{2}=10^{-4}$.

\section{Comparação entre as imagens estimadas}

Nesta seção são comparadas as imagens estimadas com e sem o ajuste das tensões. Para a análise é mostrado as imagens para $\beta^{2}=10^{-4}$, que foi considerado o melhor resultado com o ajuste da tensão. A Figura 4.23 mostra os resultados para o Caso 1 e a Figura 4.24 mostra os resultados para o Caso 2. Para os casos avaliados foi computada 


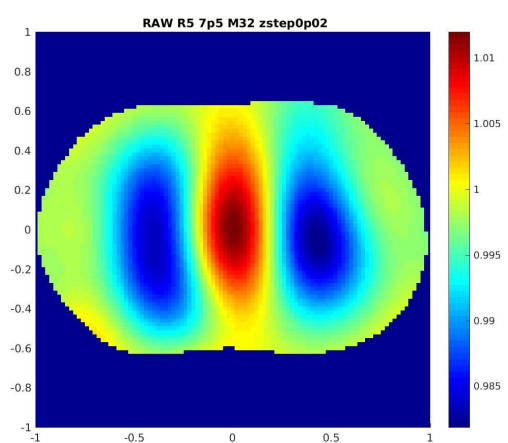

(a) Tensões inalteradas

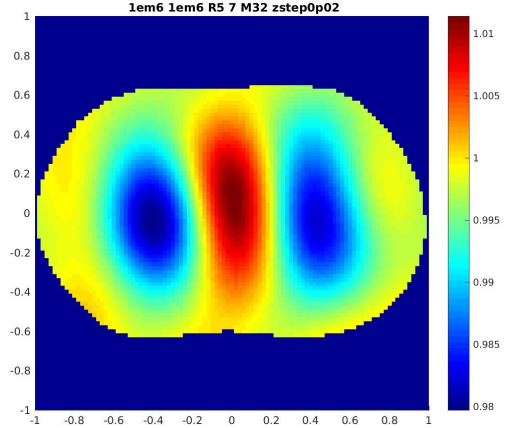

(b) $\beta^{2}=10^{-6}$

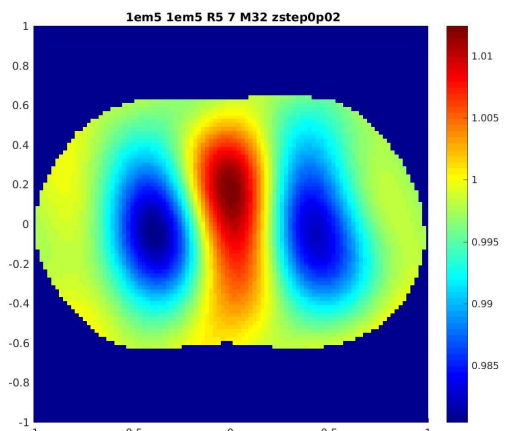

(c) $\beta^{2}=10^{-5}$

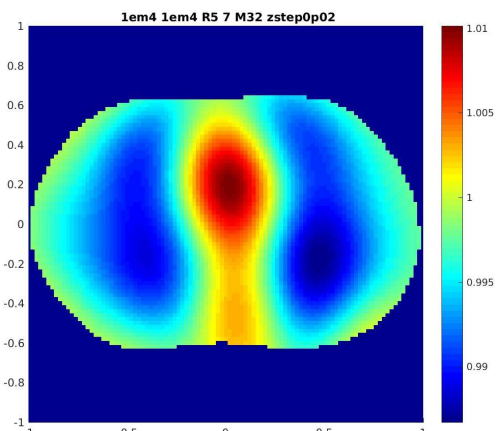

(d) $\beta^{2}=10^{-4}$

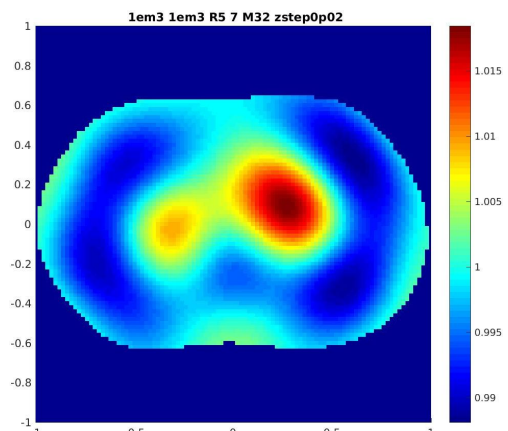

(e) $\beta^{2}=10^{-3}$

Figura 4.21: Caso 2, fantoma com cuba em formato de tórax humano com órgãos de ágar intactos.

a diferença entre a imagem estimada do fantoma onde os órgãos estavam intactos e a imagem estimada do fantoma onde parte do pulmão direito foi retirada, Figuras $4.23 c$, $4.23 \mathrm{f}, 4.24 \mathrm{c}$ e $4.24 \mathrm{f}$.

Para o Caso 1, não é possível ver o corte no pulmão, no entanto quando a imagem é comparada com o caso onde o pulmão está intacto é possível observar que a condutividade no local onde houve o corte aumentou para os dois casos. Comparando as imagens, o formato dos pulmões e do coração estão mais próximos do fantoma experimental com o uso do método do ajuste das tensões proposto. Avaliando a diferença entre as imagens, tanto as imagens reconstruída sem o ajuste das tensões quanto as imagens reconstruída com o ajuste das tensões apresentaram comportamento similares.

Para o Caso 2, é possível ver o corte no pulmão para a imagem reconstruída utilizando o método do ajuste das tensões proposto. Comparando as imagens, o formato dos pulmões e do coração estão mais próximos do fantoma experimental com o uso do método do ajuste de tensão proposto. Avaliando a diferença entre as imagens relativas, tanto as imagens reconstruída sem o ajuste das tensões quanto as imagens com o ajuste 


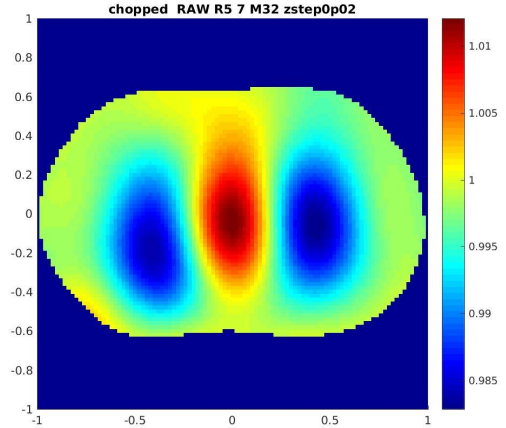

(a) Tensões inalteradas

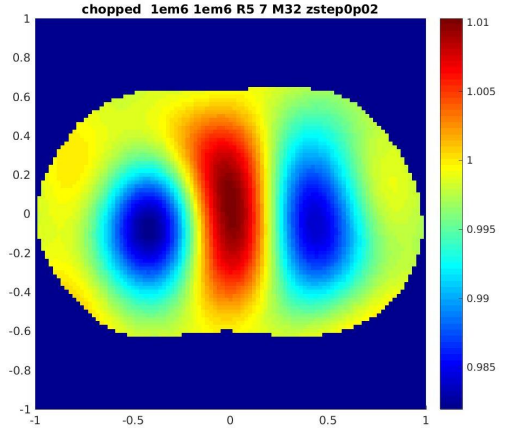

(b) $\beta^{2}=10^{-6}$

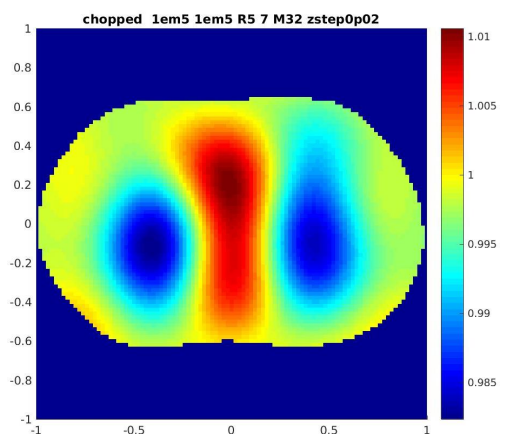

(c) $\beta^{2}=10^{-5}$

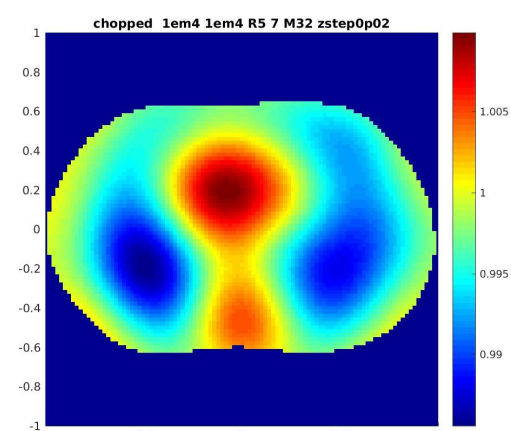

(d) $\beta^{2}=10^{-4}$

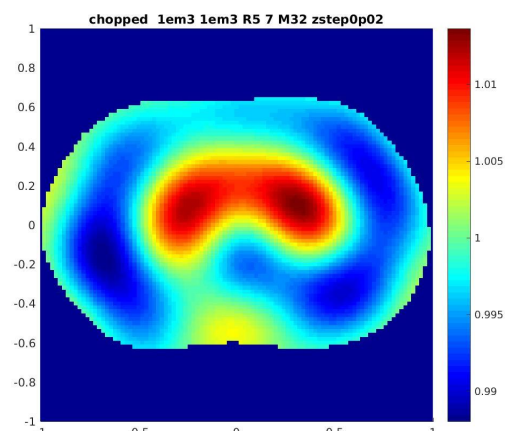

(e) $\beta^{2}=10^{-3}$

Figura 4.22: Caso 2, fantoma com cuba em formato de tórax humano com pulmão de ágar direito cortado.

das tensões apresentaram comportamento similares.

\subsection{Introdução de informação a priori através do pós- processamento das imagens reconstruídas}

Por meio de fantomas numéricos e experimentais foi avaliado o método de introdução de informação a priori através do pós-processamento das imagens reconstruídas proposto na seção 3.2. Através do método D-bar, descrito na seção 2.3.2, foram estimadas as distribuições de condutividade dos fantomas propostos e então um pósprocessamento foi aplicada à essas reconstruções.

Como primeiro passo, foi construído um prior baseado em amostras simuladas. Esse prior é composto por um conjunto de amostras de distribuições de condutividade e de suas respectivas distribuições de tensões calculadas através do problema direto simplificado.

Em seguida a distribuição de condutividade de cada uma das tensões calculadas na 


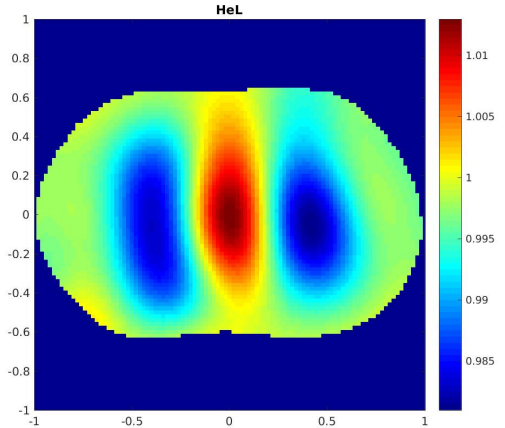

(a) Tensões inalteradas, pulmões intactos

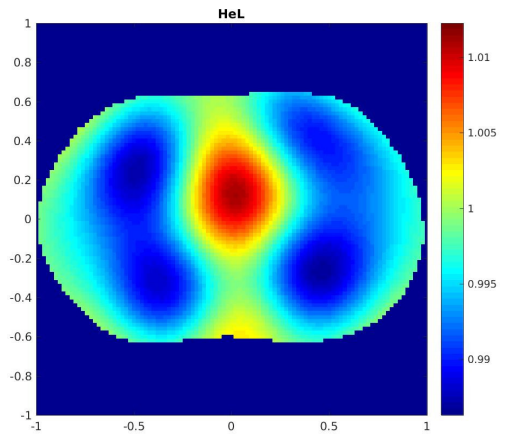

(d) $\beta^{2}=10^{-4}$, pulmões intactos

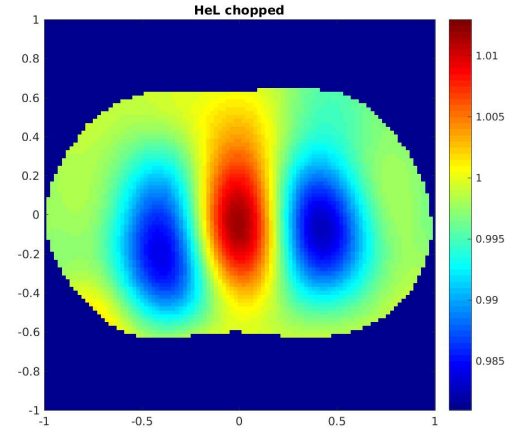

(b) Tensões inalteradas, pulmão direito cortado

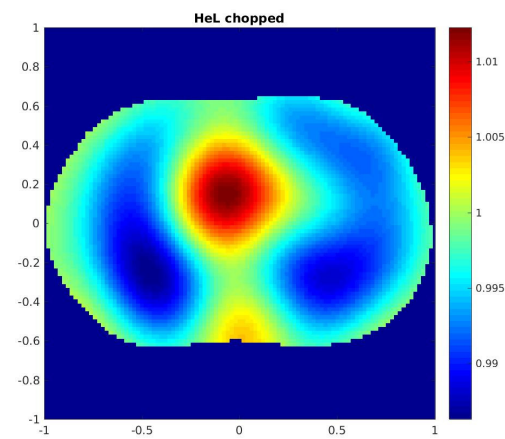

(e) $\beta^{2}=10^{-4}$, pulmão direito cortado

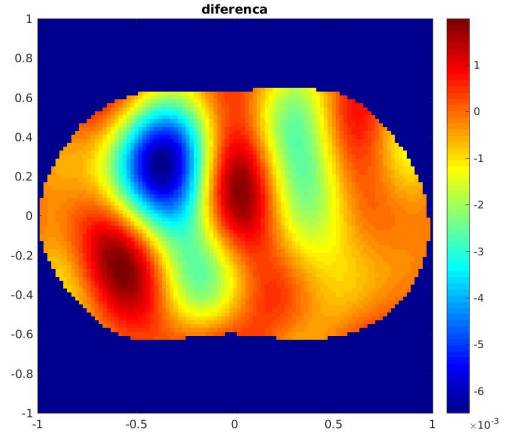

(c) Diferença entre vetores de tensão inalterados.

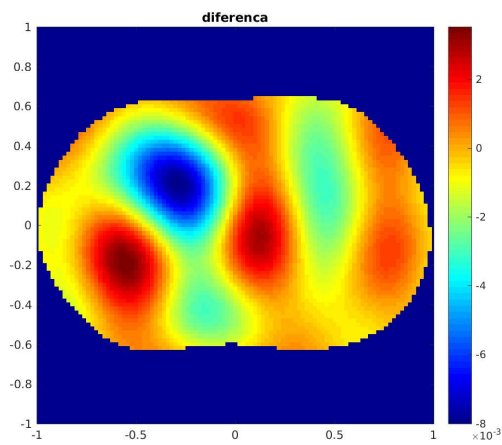

(f) Diferença entre vetores de tensão para $\beta^{2}=10^{-4}$

Figura 4.23: Caso 1, comparação dos resultados para cuba em formato de tórax humano com órgãos de ágar.

etapa anterior foi reconstruída através do método $D$-bar descrito na seção 2.3.2. Posteriormente, as distribuições de condutividade de cada uma das amostras pertencentes ao prior e de suas respectivas reconstruções foram vetorizadas.

Por fim, as imagens vetorizadas são concatenadas, conforme descrito em 3.2, e então são calculados os parâmetros A e b utilizados no pós-processamento proposto pelo método. A partir destes parâmetros, as distribuições de condutividade estimadas dos fantomas numéricos e experimentais são corrigidas e os resultados avaliados.

\subsubsection{Prior baseado em amostras simuladas}

Para que o método proposto seja avaliado, foi construído um prior baseado em amostras simuladas seguindo o mesmo processo descrito na Seção 4.3.2. Para este caso, foi utilizado como referência o contorno dos órgãos do fantoma experimental proposto adiante.

Na construção deste prior, um conjunto de $15 \mathrm{k}$ amostras simuladas de distribuições 


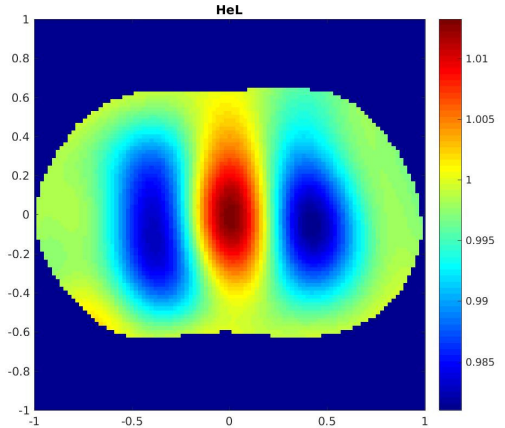

(a) Tensões inalteradas, pulmões intactos

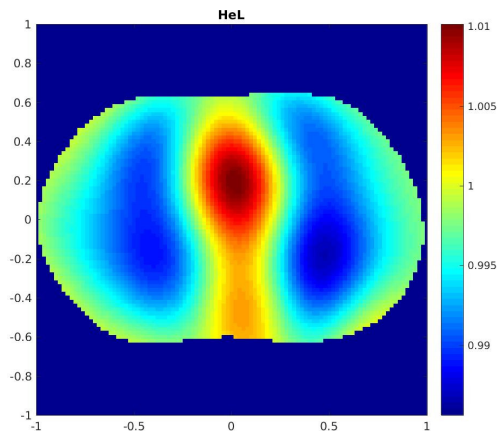

(d) $\beta^{2}=10^{-4}$, pulmões intactos

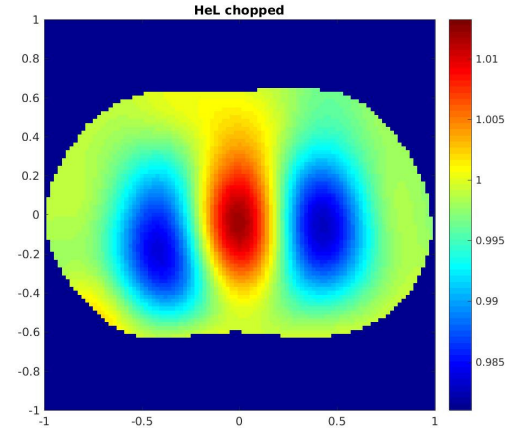

(b) Tensões inalteradas, pulmão direito cortado

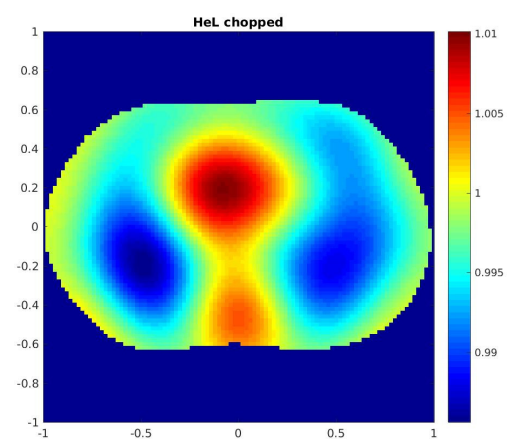

(e) $\beta^{2}=10^{-4}$, pulmão direito cortado

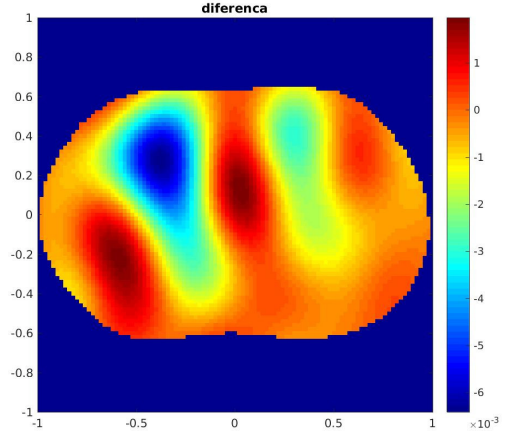

(c) Tensões inalteradas, diferença entre as imagens

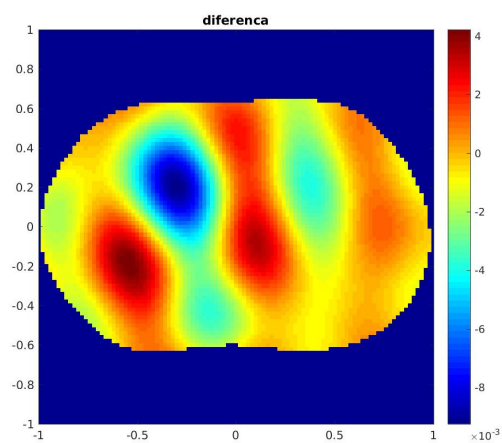

(f) Diferença entre vetores de tensão para $\beta^{2}=10^{-4}$

Figura 4.24: Caso 2, comparação dos resultados para cuba em formato de tórax humano com órgãos de ágar.

de condutividade foram geradas. Cada uma destas amostras é representada por $\sigma^{3 D}{ }_{i}$, que descreve uma distribuição de condutividade em uma malha de elementos finitos. A malha utilizada foi a mesma descrita na seção 4.3.2 e mostrada na Figura 4.16.

Em seguida, para cada $\sigma^{3 D}$, foi calculado, através do problema direto simplificado, o vetor de tensões $V\left(\sigma^{3 D}{ }_{i}\right)$. Para simular o erro de medição, é sorteado e adicionado a cada um destes vetores de tensões um vetor de ruído Gaussiano que segue a mesma média e desvio-padrão apresentado pelos eletrodos no tomógrafo ACE1. Desta forma, o ruído eletrônico de medição também é levado em consideração no ajuste das imagens.

A partir do vetor de potenciais elétricos calculado foi estimado, através do método $D$-bar descrito na Seção 2.3.2, a distribuição de condutividade $\tilde{\sigma}_{D b a r}^{2 D}$. 


\subsubsection{Cálculo dos parâmetros $\mathrm{A}$ e b}

Conforme descrito na seção 3.2, foi proposto um ajuste nas imagens reconstruídas através do método D-bar. Para este ajuste, foram calculados os parâmetros $\mathbf{A}$ e $\mathbf{b}$ propostos na equação 3.17 .

Através de uma interpolação Gaussiana as distribuições de condutividade $\sigma^{3 D_{i}}$ são transformadas em imagens $\sigma^{2 D}{ }_{i}$. Estas imagem representam a distribuição de condutividade na seção transversal onde a cinta com os eletrodos está posicionada. Neste experimento, utilizou-se uma interpolação Gaussiana, descrita através da matriz $G \in \mathbb{R}^{n \_p i x e l \times n \_t e t r a}$, que calcula uma imagem pixelada de tamanho $64 \times 64$ (n_pixel) a partir dos $13 \mathrm{k}$ elementos tetraédricos (n_tetra) da malha de elementos finitos, equação 4.2 .

$$
\sigma^{2 D}{ }_{i}=G \sigma^{3 D}
$$

A partir do conjunto de amostas das imagens $\sigma^{2 D}{ }_{i}$ e de suas respectivas distribuições de condutividades $\tilde{\sigma}_{D b a r}^{2 D}$, é construído o conjunto de vetores $z_{i}$ descritos pela equação 3.15. Neste caso, $\sigma^{2 D}{ }_{i}$ é representado através da variável $x_{i}$ e $\tilde{\sigma}_{D b a r ~}^{2 D}$ através da variável $y_{i}$.

Em seguida, foi calculada a expectância $\mu_{z}$ e a matriz de covariância $\Gamma_{z}$ do conjunto de amostras $z$. Conforme mostrado na equação 3.16, foram obtidas as expectâncias $\mu_{x}$ e $\mu_{y}$, as matrizes de covariância $\Gamma_{x x}$ e $\Gamma_{y y}$ e as matrizes de covariância cruzada $\Gamma_{x y}$ e $\Gamma_{y x}$ relacionadas aos conjuntos de amostras $\sigma^{2 D}{ }_{i}$ e $\tilde{\sigma}_{D b a r}^{2 D}$.

Por fim, a partir das expectâncias, matrizes de covariância e covariância cruzadas a matriz $\mathbf{A}$ e o vetor $\mathbf{b}$ foram calculados segundo a equação 3.17. Vale observar que para o cálculo dos parâmetros $\mathbf{A}$ e $\mathbf{b}$ é necessário inverter a matriz $\Gamma_{y y}$. Esta matriz representa a matriz de covariância do conjunto de amostras do prior simulado e geralmente é mal-condicionada. Para que $\Gamma_{y y}$ seja não-singular e inversível, um ruído branco é adicionado à sua diagonal,

$$
\Gamma_{y y}^{-1}=\left(\Gamma_{y y}+\kappa I\right)^{-1}
$$

onde $\kappa$ é o parâmetro que controla o nível de ruído branco e $I$ a matriz de identidade. 


\subsubsection{Fantoma numérico}

Para avaliar o desempenho do método de introdução de informação a priori através do pós-processamento imagens reconstruídas fantomas numéricos foram simulados. Através de um problema direto refinado (51 k elementos tetraédricos), calculou-se vetores de potenciais elétricos para algumas distribuições de condutividade. Posteriormente as imagens foram reconstruídas a partir do método D-bar descrito em 2.3.2 e então corrigidas através do método avaliado nesta seção.

Para calcular o problema direto, foi utilizado um valor de impedância de contato $10 \%$ menor que o valor utilizado para construir o prior baseado em amostras simuladas e para a capacitância parasita utilizou-se um valor 10\% maior. Para simular o erro de medição, é sorteado e adicionado a cada uma das tensões dos fantomas numéricos um vetor de ruído Gaussiano que segue a mesma média e desvio-padrão apresentado pelos eletrodos no tomógrafo ACE1. Como tensão de referência foi utilizado a tensão calculada para a cuba homogênea com condutividade da solução salina em $0.19 \mathrm{~S} / \mathrm{m}$.

Para cada uma das Figuras 4.25-4.34 é mostrado as distribuições de condutividade simuladas ( $1^{a}$ linha), as imagens reconstruídas através do método $D$-bar ( $2^{a}$ linha) e as imagens corrigidas através do método proposto ( $3^{a}$ linha). Na primeira coluna são apresentadas as imagens de referência, com o contorno médio. Na segunda coluna estão dispostas as figuras dos fantomas simulados. E na última coluna foi computado a diferença entre as duas primeiras colunas.

A Figura 4.25 mostra um fantoma onde foi removido um dos pulmões, as Figuras 4.26, 4.27 e 4.28 mostram fantomas onde foram provocadas rotações nos pulmões. Para as Figuras 4.29, 4.30 e 4.31 foram provocados diferentes tamanhos de corte na base de um dos pulmões, enquanto as Figuras 4.32 e 4.33 mostram simulações de corte no topo de um dos pulmões. Por fim, a Figura 4.34 mostra um caso onde houve a redução do tamanho do pulmão esquerdo.

Diversos fantomas foram simulados, provocando diferentes deformações no domínio. Para todos os casos simulados o método proposto melhorou significativamente a resolução espacial das imagens. Mesmo com a diferença de 10\% na impedância de contato e na capacitância parasita dos eletrodos e utilizando uma malha mais refinada na geração das tensões o método apresentou resultado satisfatório. Para o caso 4.32 o corte foi superestimado pelo pós-processamento e foi introduzido um artefato no 


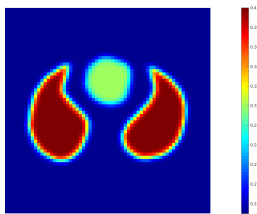

(a) Referência, simulada

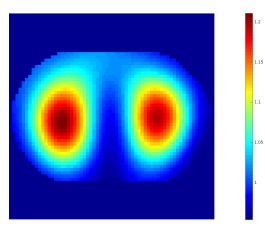

(d) Referência, reconstruída

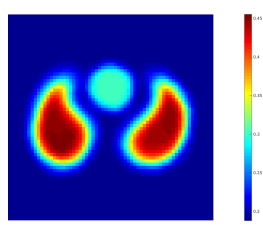

(g) Referência, corrigida

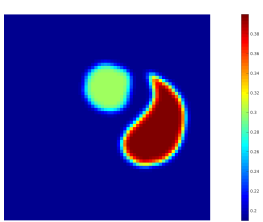

(b) Fantoma simulado

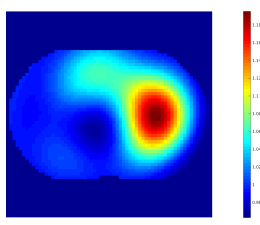

(e) Fantoma reconstruído

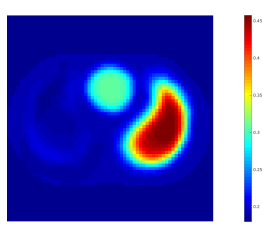

(h) Fantoma corrigido

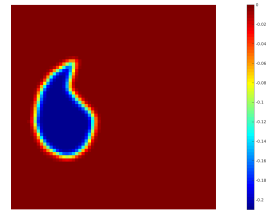

(c) Diferença, simulado

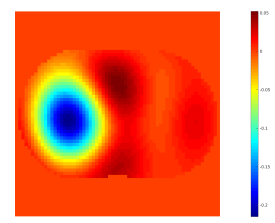

(f) Diferença, reconstruído

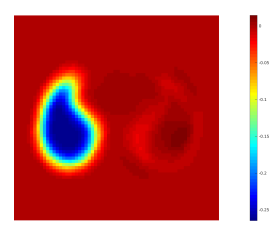

(i) Diferença, corrigido

Figura 4.25: Caso 1, remoção do pulmão direito.

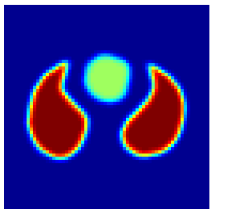

(a) Referência, simulada

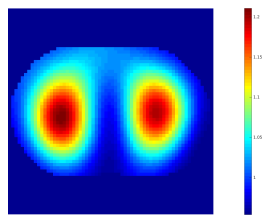

(d) Referência, reconstruída

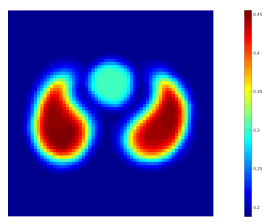

(g) Referência, corrigida

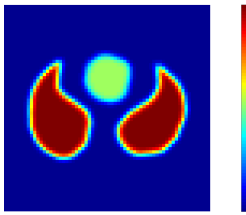

(b) Fantoma simulado

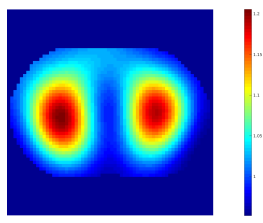

(e) Fantoma reconstruído

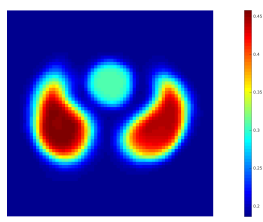

(h) Fantoma corrigido

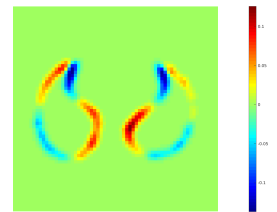

(c) Diferença, simulado

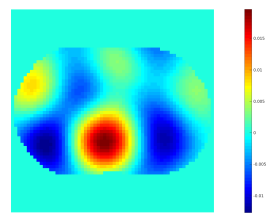

(f) Diferença, reconstruído

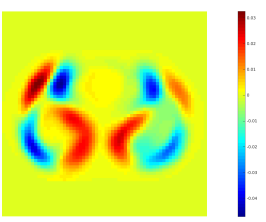

(i) Diferença, corrigido

Figura 4.26: Caso 2, rotação dos pulmões para fora. 


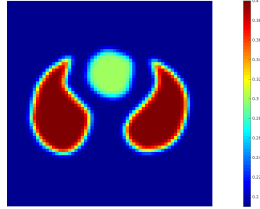

(a) Referência, simulada

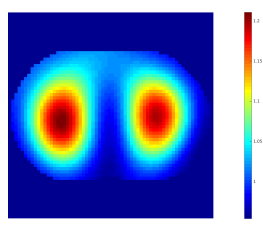

(d) Referência, reconstruída

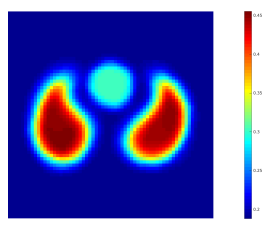

(g) Referência corrigida

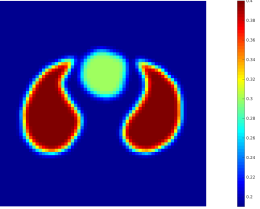

(b) Fantoma simulado

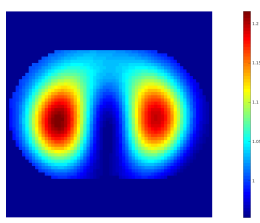

(e) Fantoma reconstruído

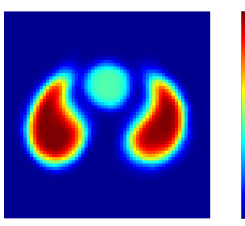

(h) Fantoma corrigido

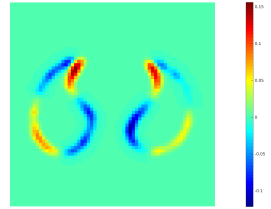

(c) Diferença, simulado

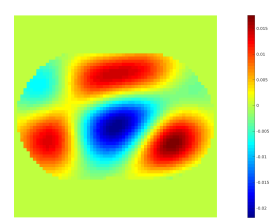

(f) Diferença, reconstruído

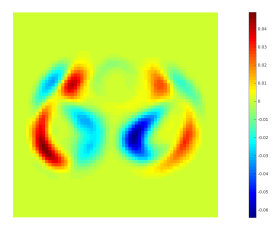

(i) Diferença, corrigido

Figura 4.27: Caso 3, rotação dos pulmões para dentro.

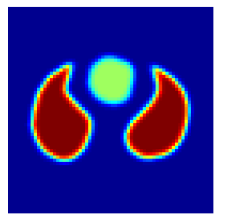

(a) Referência, simulada

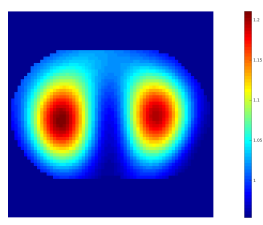

(d) Referência, reconstruída

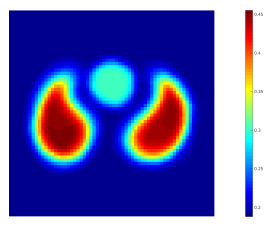

(g) Referência, corrigida

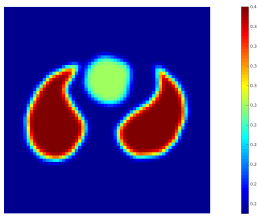

(b) Fantoma simulado

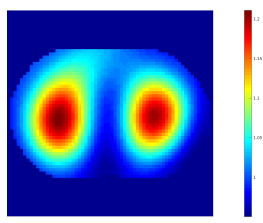

(e) Fantoma reconstruído

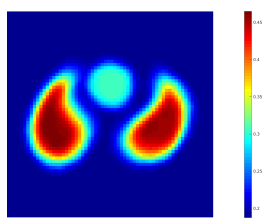

(h) Fantoma corrigido

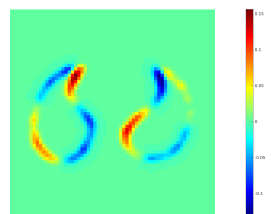

(c) Diferença, simulado

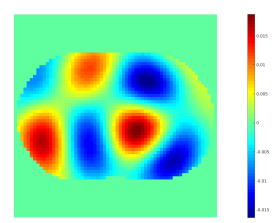

(f) Diferença, reconstruído

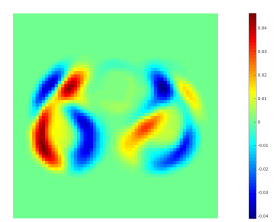

(i) Diferença, corrigido

Figura 4.28: Caso 4, rotação pulmão direito para dentro e pulmão esquerdo para fora. 


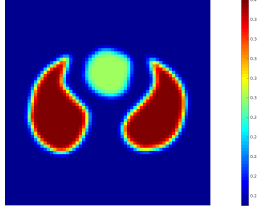

(a) Referência, simulada

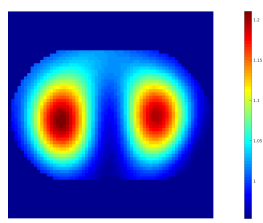

(d) Referência, reconstruída

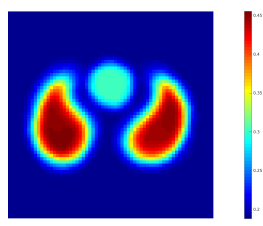

(g) Referência, corrigida

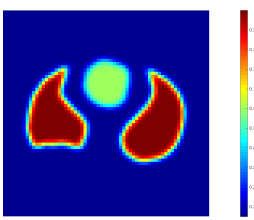

(b) Fantoma simulado

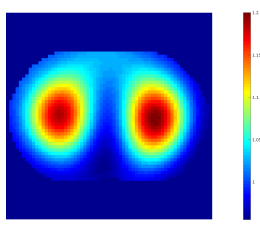

(e) Fantoma reconstruído

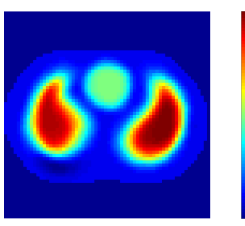

(h) Fantoma corrigido

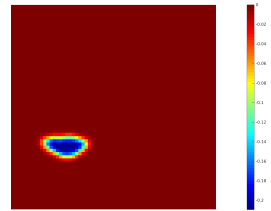

(c) Diferença, simulado

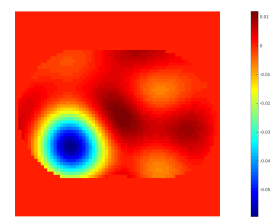

(f) Diferença, reconstruído

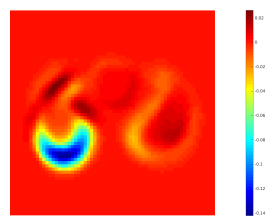

(i) Diferença, corrigido

Figura 4.29: Caso 5, pequeno corte na base do pulmão direito.

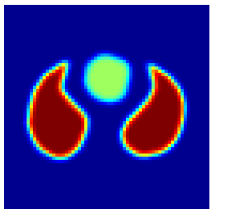

(a) Referência simulada

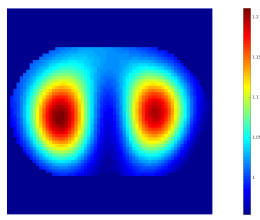

(d) Referência, reconstruída

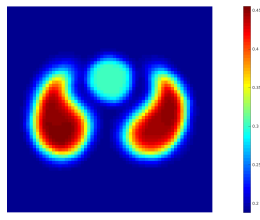

(g) Referência, corrigida

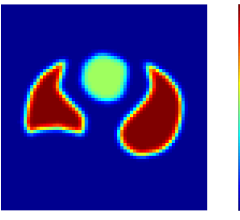

(b) Fantoma simulado

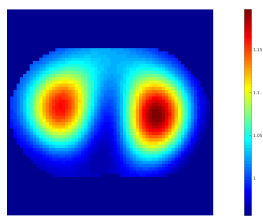

(e) Fantoma reconstruído

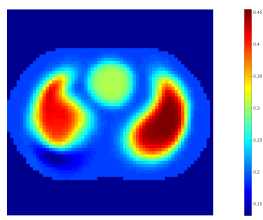

(h) Fantoma corrigido

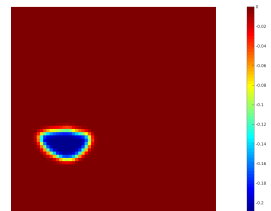

(c) Diferença, simulado

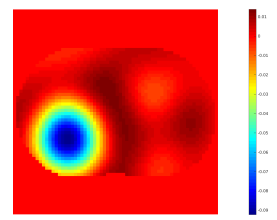

(f) Diferença, reconstruído

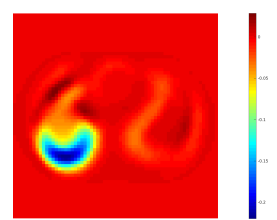

(i) Diferença, corrigido

Figura 4.30: Caso 6, corte médio na base do pulmão direito. 


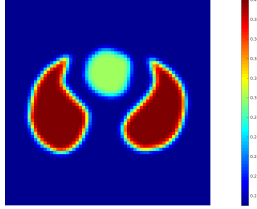

(a) Referência, simulada

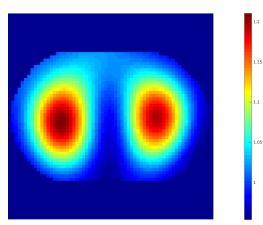

(d) Referência, reconstruída

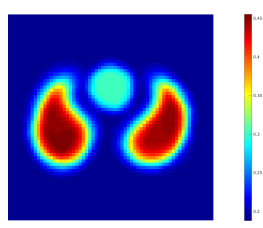

(g) Referência, corrigida

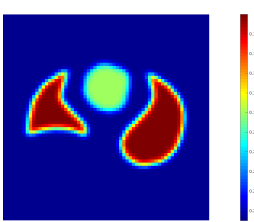

(b) Fantoma simulado

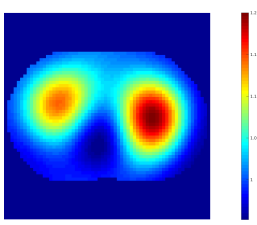

(e) Fantoma reconstruído

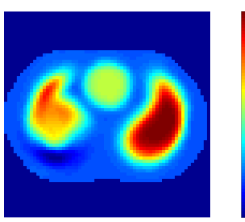

(h) Fantoma corrigido

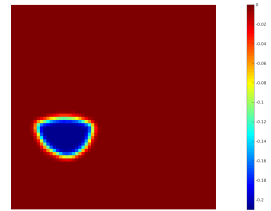

(c) Diferença, simulado

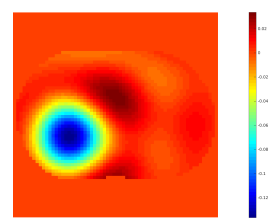

(f) Diferença, reconstruído

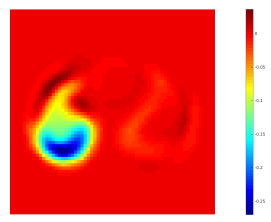

(i) Diferença, corrigido

Figura 4.31: Caso 7, corte grande na base do pulmão direito.

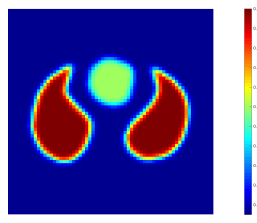

(a) Referência, simulada

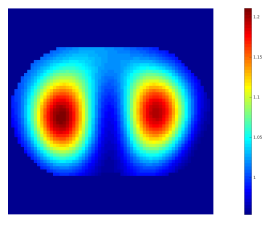

(d) Referência, reconstruída

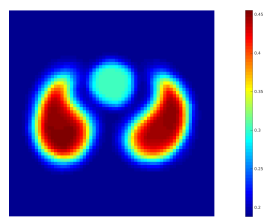

(g) Referência, corrigida

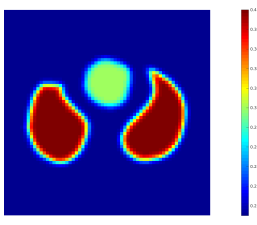

(b) Fantoma simulado

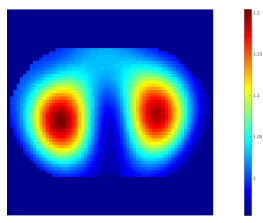

(e) Fantoma reconstruído

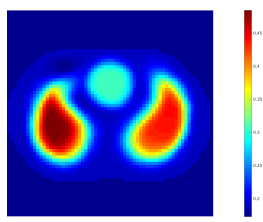

(h) Fantoma corrigido

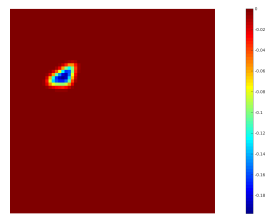

(c) Diferença, simulado

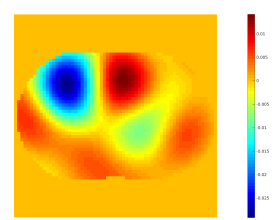

(f) Diferença, reconstruído

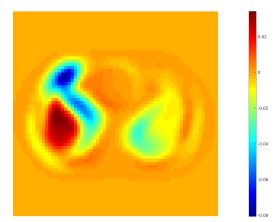

(i) Diferença, corrigido

Figura 4.32: Caso 8, pequeno corte topo do pulmão direito. 


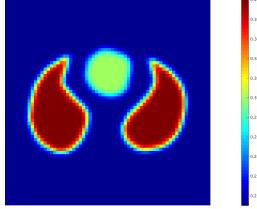

(a) Referência, simulada

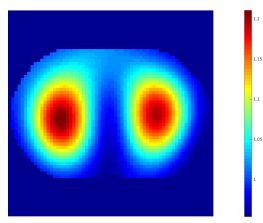

(d) Referência, reconstruída

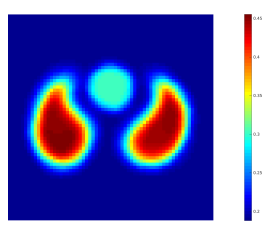

(g) Referência, corrigida

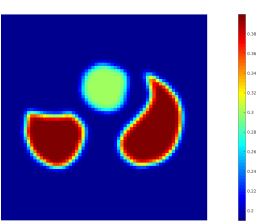

(b) Fantoma simulado

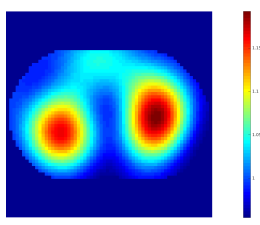

(e) Fantoma reconstruído

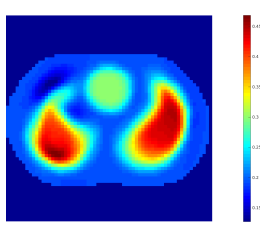

(h) Fantoma corrigido

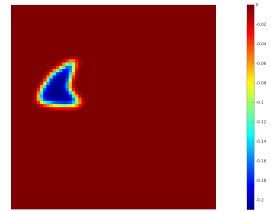

(c) Diferença, simulado

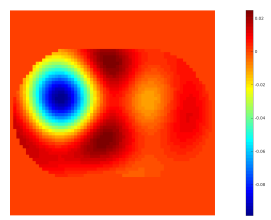

(f) Diferença, reconstruído

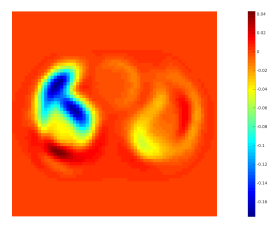

(i) Diferença, corrigido

Figura 4.33: Caso 9, corte grande no topo do pulmão direito.

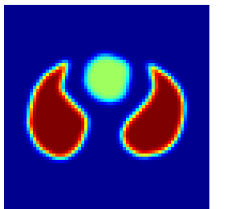

(a) Referência simulada

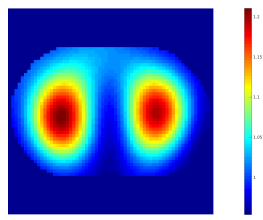

(d) Referência, reconstruída

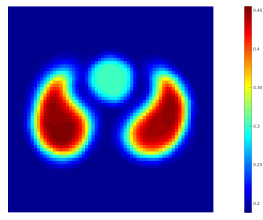

(g) Referência, corrigida

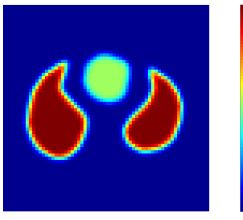

(b) Fantoma simulado

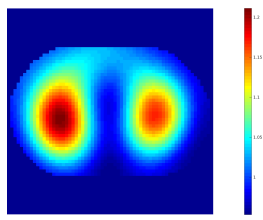

(e) Fantoma reconstruído

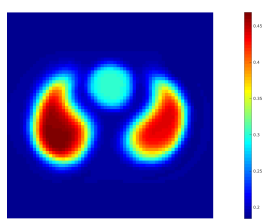

(h) Fantoma corrigido

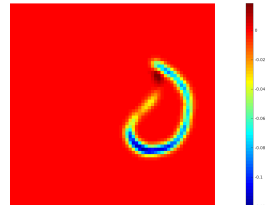

(c) Diferença, simulado

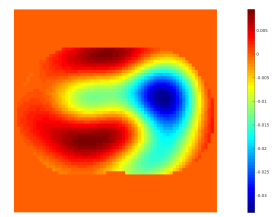

(f) Diferença, reconstruído

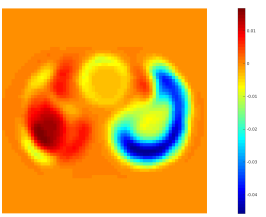

(i) Diferença, corrigido

Figura 4.34: Caso 10, redução tamanho do pulmão esquerdo. 
pulmão intacto.

\subsubsection{Fantoma experimental}

A Figura 4.35 mostra o fantoma experimental proposto para avaliar a introdução de informação a priori através do pós-processamento das imagens reconstruídas. A partir das tensões medidas na cuba, as distribuições de condutividade de cada caso foram estimadas utilizando o método D-bar. Posteriormente, essas distribuições foram corrigidas através do método proposto na seção 3.2 e os resultados obtidos foram avaliados.

Neste fantoma experimental, foram preparados dois objetos cortados do interior de uma melancia e um objeto a base de ágar. Ajustou-se a condutividade da peça de ágar que representa o coração para condutividade próxima à esperada deste órgão e mediuse no final do experimento a condutividade das peças de melancia, que representam os pulmões. A cuba foi preenchida com solução salina e os objetos foram cortados e posicionados no centro da cuba, simulando a anatomia e posição do coração e dos pulmões.

A cuba de acrílico tem o formato de um tórax humano e apresenta 32 eletrodos (25 mm de largura) no seu entorno. Para este experimento foi utilizada uma solução salina de condutividade $0.20 \mathrm{~S} / \mathrm{m}$, a peça de ágar com condutividade $0.38 \mathrm{~S} / \mathrm{m}$ e duas peças de melancia com condutividades $0.15 \mathrm{~S} / \mathrm{m}$. A medição da propriedade elétrica de cada um dos órgãos e da solução salina foi realizada através de um condutivímetro modelo Omega CDH221.

Primeiramente realizou-se a medição das tensões na cuba em formato de tórax humano preenchida apenas com solução salina. Em um segundo momento, os objetos feitos de ágar e melancia foram inseridos na cuba e novas medições foram adquiridas. Em seguida, foi realizado um corte e retirada a parte inferior da peça de melancia que representava o pulmão direito. Depois, retirou-se uma parte ainda maior na mesma peça de melancia. Para as medições, utilizou-se o tomógrafo ACE1 ajustado para excitar o domínio através do padrão de corrente pula-0. 


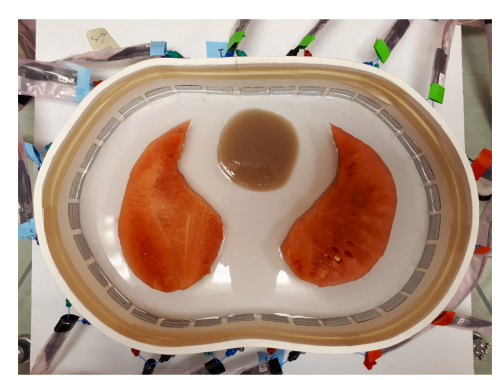

(a) Coração e pulmões intactos

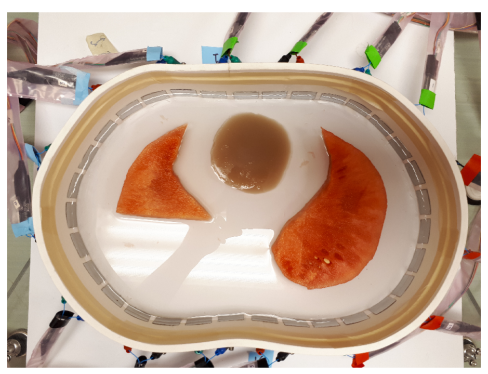

(c) Pulmão direito, corte grande

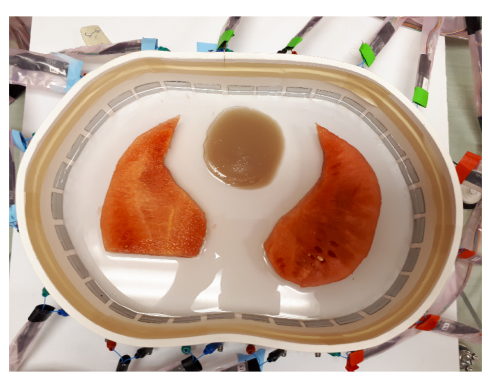

(b) Pulmão direito, corte pequeno

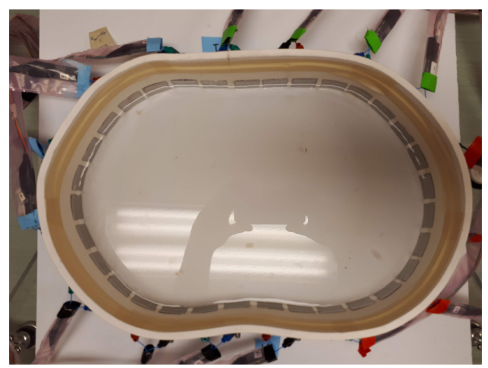

(d) Meio homogêneo

Figura 4.35: Fantoma experimental, cuba em formato de tórax humano com coração de ágar e pulmões de melancia.

\section{Imagens reconstruídas}

Para cada uma das tensões medidas do fantoma proposto, dois conjuntos de dados foram estimados através do algoritmo D-bar descrito na Seção 2.3.2. Para o primeiro conjunto de dados, foram estimadas as distribuições de condutividade utilizando como tensão de referência o problema direto simplificado corrigido pelo erro de aproximação. E para o segundo conjunto de dados, foram estimadas novamente as distribuições de condutividade utilizando como tensão de referência a tensão medida na cuba em formato de tórax humano quando esta estava preenchida apenas com a solução salina.

\section{Imagens absolutas}

A Figura 4.36 mostra as imagens estimadas através do método $D$-bar a partir das tensões medidas do fantoma experimental proposto. A Figura 4.36a mostra a reconstrução quando o coração de ágar e os pulmões de melancia estavam intactos, enquanto a Figura 4.36 b mostra a distribuição de condutividade quando houve o primeiro corte 
e a Figura 4.36c mostra o resultado quando o corte foi ampliado.

Para a reconstrução destas imagens foi utilizado como tensão de referência um vetor de tensões calculado a partir da malha mostrada na Figura 4.16. Foi utilizado o método proposto na Seção 3.3.1 para encontrar a estimativa inicial e calcular o erro de aproximação. No problema direto refinado foi utilizada uma malha de $91 \mathrm{k}$ elementos tetraédricos.

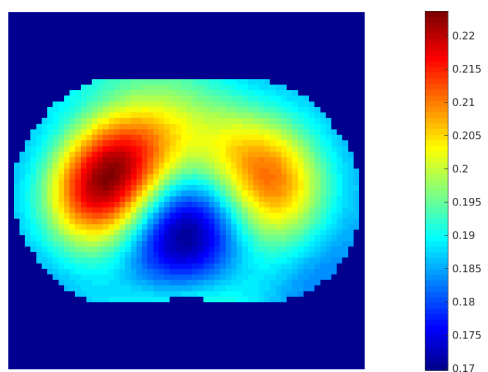

(a) Órgãos intactos

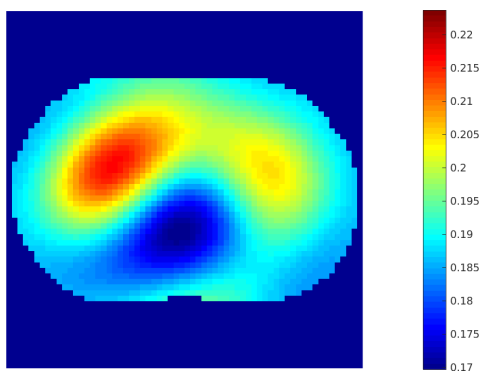

(b) Pulmão direito, corte pequeno

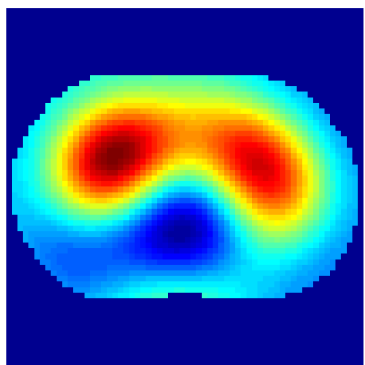

(c) Pulmão direito, corte grande

Figura 4.36: Imagens absolutas, cuba em formato de tórax humano com coração de ágar e pulmão de melancia.

A Figura 4.37 mostra os resultados obtidos utilizando o método proposto para introduzir informação a priori através do pós-processamento das imagens absolutas reconstruídas. A partir das expectâncias $\mu_{x}$ e $\mu_{y}$, das matrizes de covariância $\Gamma_{x x}$ e $\Gamma_{y y}$ e das matrizes de covariância cruzada $\Gamma_{x y}$ e $\Gamma_{y x}$ obtidas através do método proposto, foram calculadas a matriz $\mathbf{A}$ e o vetor $\mathbf{b}$ para 3 valores distintos do parâmetro $\kappa$. Na primeira linha são mostrados os resultados das reconstruções corrigidas quando o parâmetro que controla o nível de ruído branco para a inversão da matriz de covariância $\Gamma_{y y}$ foi de $10^{-2}$, enquanto na segunda e terceira linhas estão os resultado para $\kappa=10^{-4}$ e $10^{-8}$, respectivamente.

Para os diversos valores de $\kappa$ foram calculadas as diferenças entre as imagens absolutas estimadas do fantoma onde os órgãos estavam intactos e as imagens absolutas estimadas do fantoma onde parte do pulmão direito foi retirada. A Figura 4.38 mostra a diferença entre as imagens para o primeiro corte realizado, enquanto a Figura 4.39 mostra a diferença entre as imagens para o corte amplificado.

A Figura 4.36 mostrou o resultado da distribuição de condutividade absoluta estimada utilizando o método D-bar. Através destas imagens não é possível observar a posição e formato do coração. Comparando as imagens estimadas também não é 


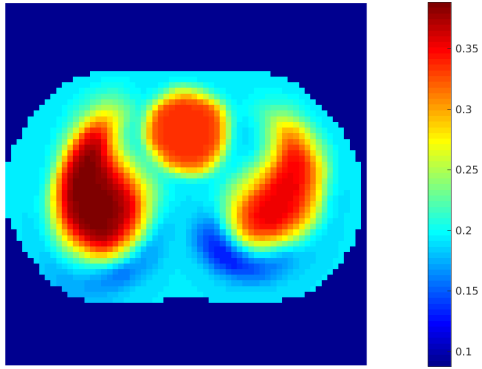

(a) Órgãos intactos, $\kappa=10^{-2}$

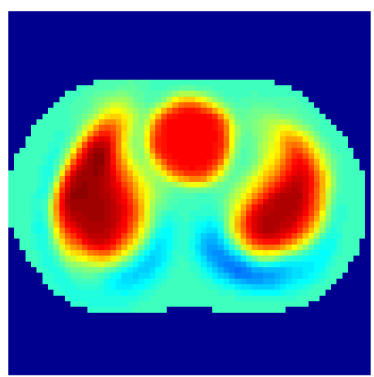

(d) Órgãos intactos, $\kappa=10^{-4}$

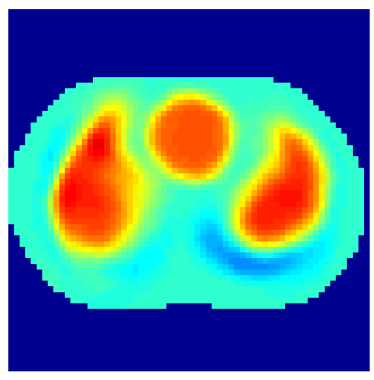

(g) Órgãos intactos, $\kappa=10^{-8}$

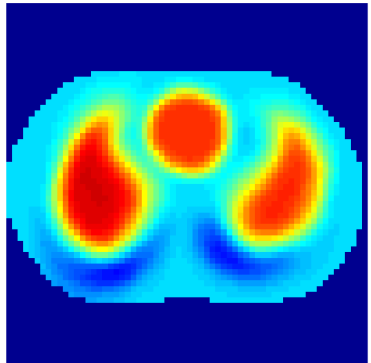

(b) Pulmão direito, corte pequeno, $\kappa=10^{-2}$

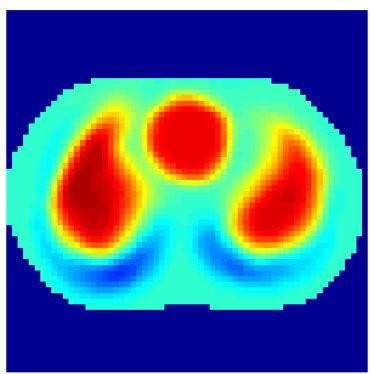

(e) Pulmão direito, corte pequeno, $\kappa=10^{-4}$

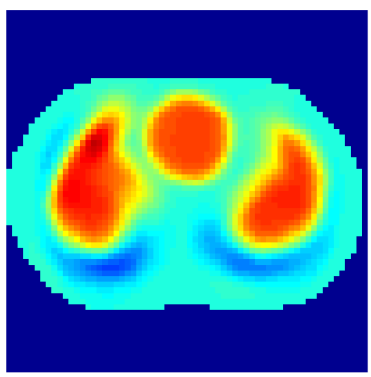

(h) Pulmão direito, corte pequeno, $\kappa=10^{-8}$

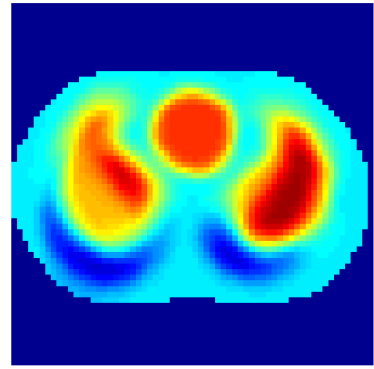

(c) Pulmão direito, grande, $\kappa=10^{-2}$

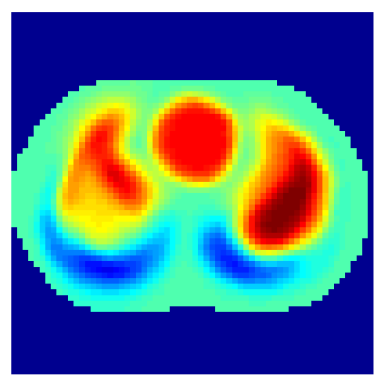

(f) Pulmão direito, grande, $\kappa=10^{-4}$

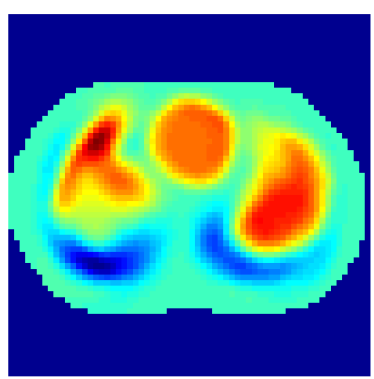

(i) Pulmão direito, corte grande, $\kappa=10^{-8}$

Figura 4.37: Imagens absolutas, cuba em formato de tórax humano com coração de ágar e pulmão de melancia com o pós-processamento das imagens.

possível determinar a localização dos cortes. Para a imagem que representa o fantoma com o maior corte no pulmão houve uma união entre os pulmões na posição do coração. Por fim, as imagens apresentaram um artefato de condutividade baixa entre os pulmões e os contornos dos órgãos não puderam ser determinados.

Com o ajuste das imagens absolutas através do método proposto, Figura 4.36, a resolução espacial das imagens melhorou consideravelmente. As imagens pósprocessadas apresentaram formato e posição dos órgãos próximos à posição mostrada no fantoma. É possível distinguir onde o corte ocorreu apenas para as imagens onde houve o maior corte no pulmão. Comparando as imagens para os diversos valores do parâmetro $\kappa$ é possível observar que os cortes no pulmão ficaram mais evidentes nos 

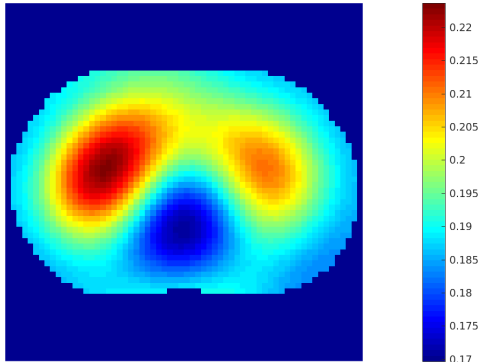

(a) Órgãos intactos, sem ajuste

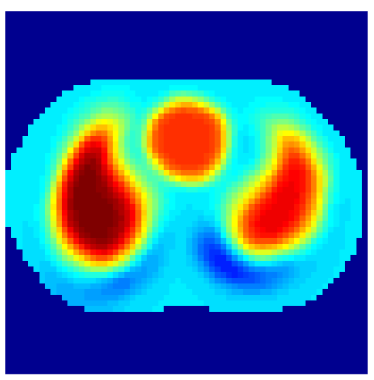

(d) Órgãos intactos, $\kappa=10^{-2}$

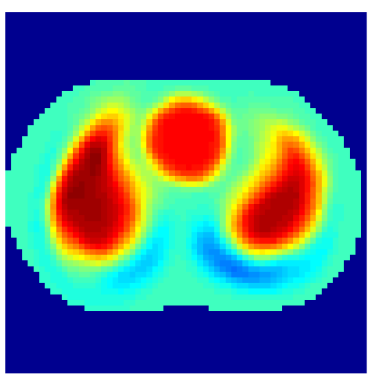

(g) Órgãos intactos, $\kappa=10^{-4}$

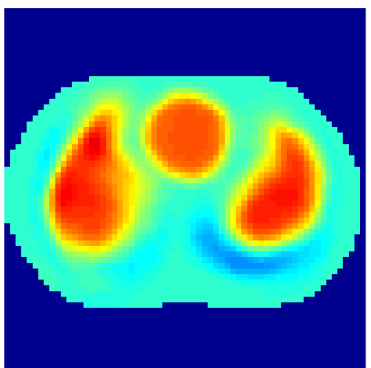

(j) Órgãos intactos, $\kappa=10^{-8}$

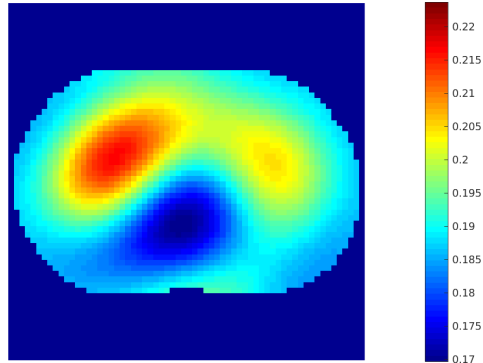

(b) Pulmão direito, corte pequeno, sem ajuste

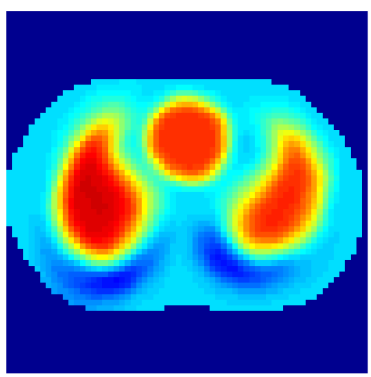

(e) Pulmão direito, corte pequeno, $\kappa=10^{-2}$

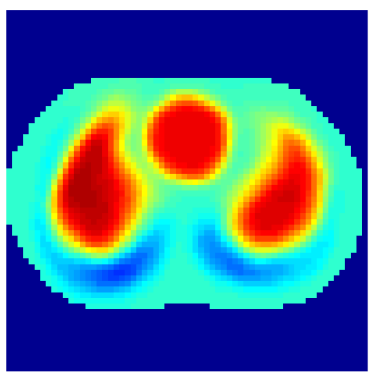

(h) Pulmão direito, corte pequeno, $\kappa=10^{-4}$
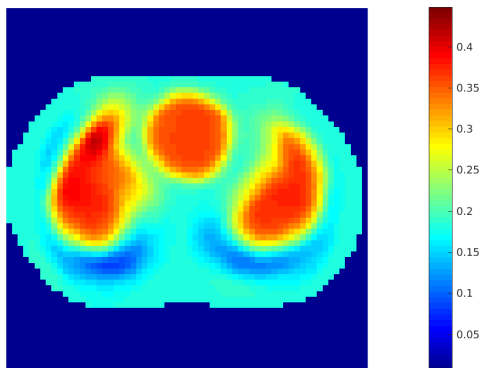

(k) Pulmão direito, corte pequeno, $\kappa=10^{-8}$

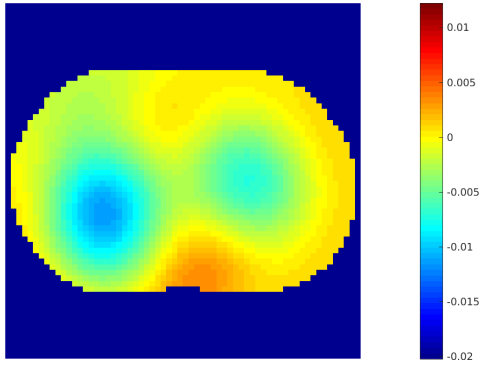

(c) Diferença, corte pequeno, sem ajuste

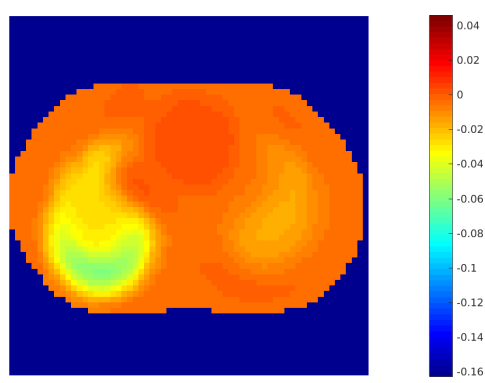

(f) Diferença, corte pequeno, $\kappa=10^{-2}$

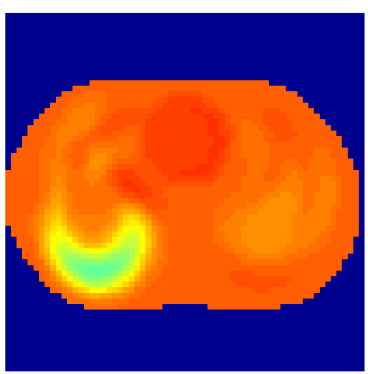

(i) Diferença, corte pequeno, $\kappa=10^{-4}$

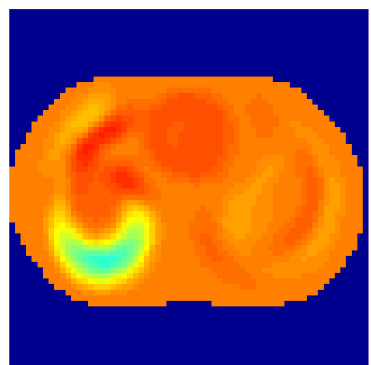

(1) Diferença, corte pequeno, $\kappa=10^{-8}$

Figura 4.38: Diferença entre imagens absolutas para corte pequeno no pulmão direito, cuba em formato de tórax humano com coração de ágar e pulmão de melancia com o pós-processamento das imagens.

casos onde o parâmetro utilizado foi menor. No entanto, quanto menor foi o parâmetro $\kappa$ maior o artefato próximo à base dos pulmões. 


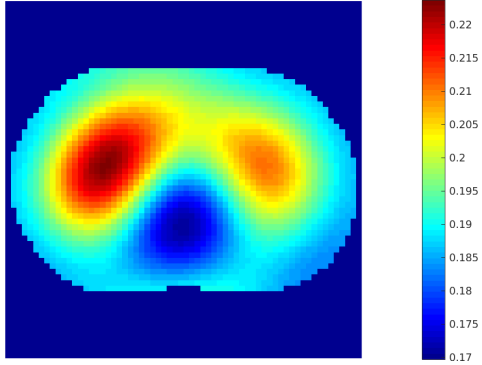

(a) Órgãos intactos, sem ajuste

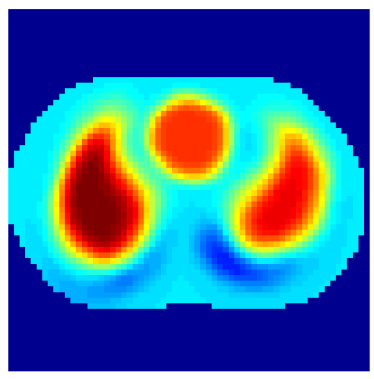

(d) Órgãos intactos, $\kappa=10^{-2}$

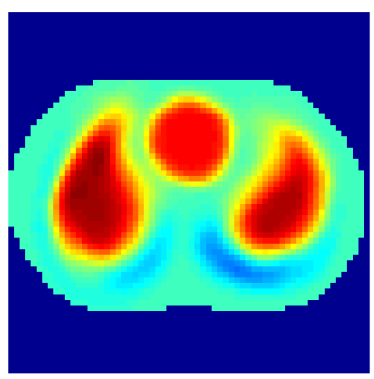

(g) Órgãos intactos, $\kappa=10^{-4}$

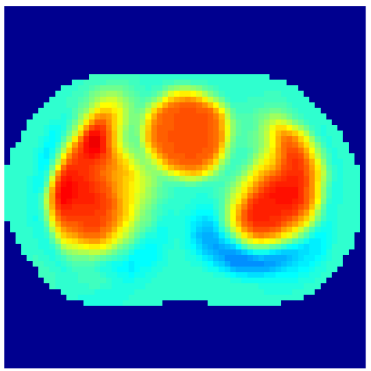

(j) Órgãos intactos, $\kappa=10^{-8}$

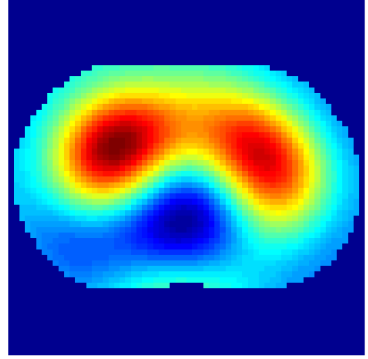

(b) Pulmão direito, grande, sem ajuste

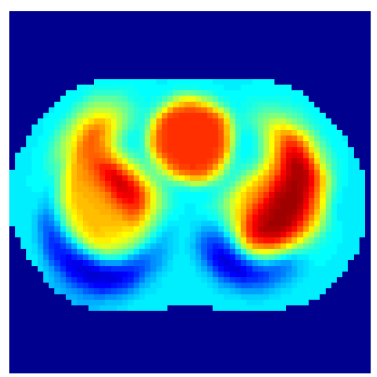

(e) Pulmão direito, grande, $\kappa=10^{-2}$

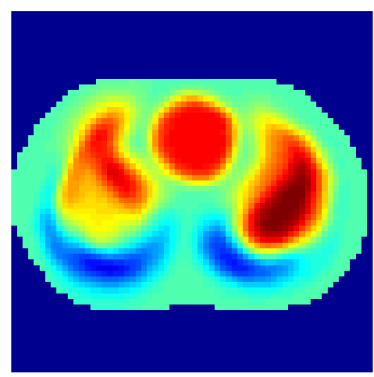

(h) Pulmão direito, grande, $\kappa=10^{-4}$

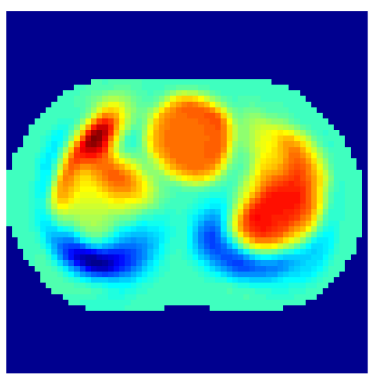

(k) Pulmão direito, grande, $\kappa=10^{-8}$

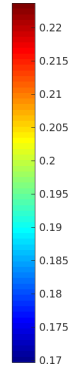

corte

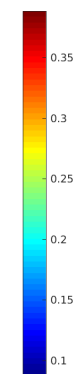

corte

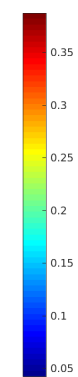

corte

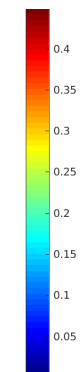

corte

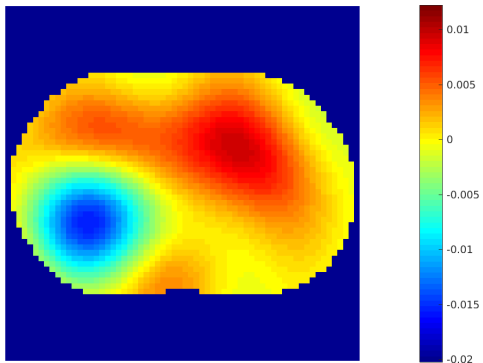

(c) Diferença, corte grande, sem ajuste

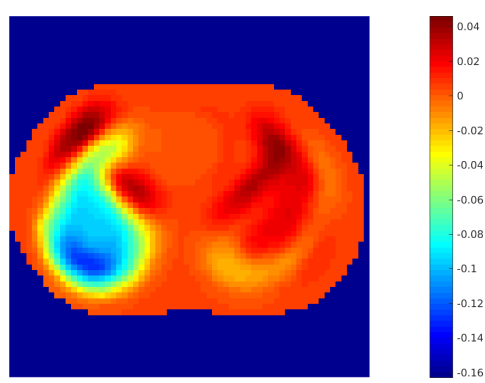

(f) Diferença, corte grande, $\kappa=$ $10^{-2}$

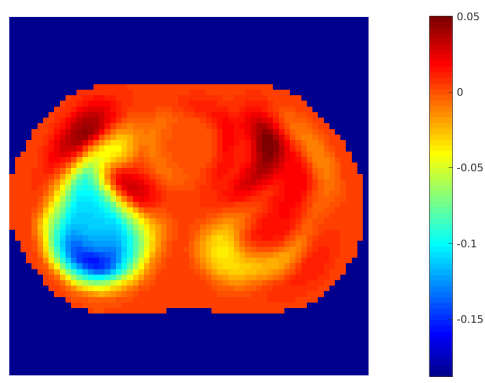

(i) Diferença, corte grande, $\kappa=$ $10^{-4}$

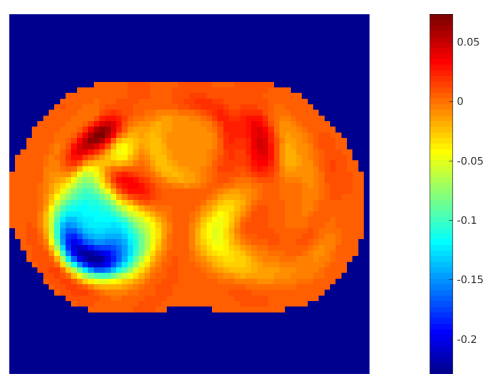

(1) Diferença, corte grande, $\kappa=$ $10^{-8}$

Figura 4.39: Diferença entre imagens absolutas para corte grande no pulmão direito, cuba em formato de tórax humano com coração de ágar e pulmão de melancia com pós-processamento das imagens.

As figuras 4.38 e 4.39 mostram a diferença entre as reconstruções quando o pulmão direito estava intacto e quando cortado. Através destas imagens é possível perceber 
que tanto o corte pequeno quanto o corte grande foram melhor identificados com o uso do método proposto. Comparando as imagens para os diversos valores do parâmetro $\kappa$ é possível observar que quanto maior o seu valor, mais rígido o método proposto se torna, isto é, mais próximo da distribuição esperada a priori as imagens ficam.

\section{Imagens Relativas}

A Figura 4.40 mostra as imagens estimadas através do método $D$-bar a partir das tensões medidas do fantoma experimental proposto e utilizando o vetor de tensões medido para o caso homogêneo como tensão de referência. A Figura 4.40a mostra a reconstrução quando o coração de ágar e os pulmões de melancia estavam intactos, enquanto a Figura 4.40b mostra a distribuição de condutividade quando houve o primeiro corte e a Figura 4.40c mostra o resultado quando o corte foi ampliado.

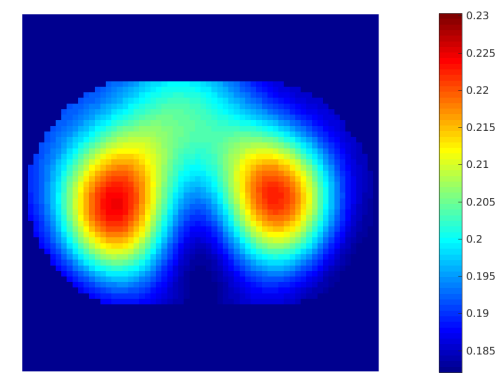

(a) Órgãos intactos

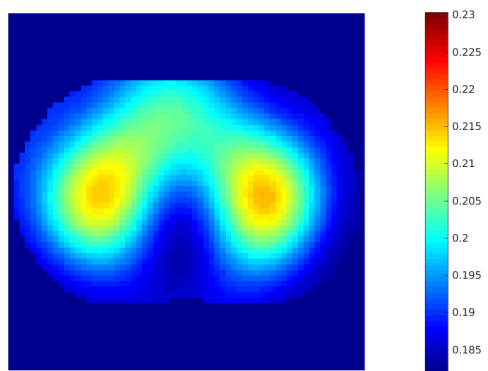

(b) Pulmão direito, corte pequeno

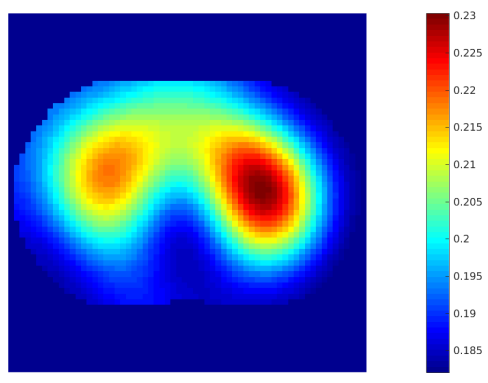

(c) Pulmão direito, corte grande

Figura 4.40: Imagens relativas, cuba em formato de tórax humano com coração de ágar e pulmão de melancia.

A Figura 4.41 mostra os resultados obtidos utilizando o método proposto para introduzir informação a priori através do pós-processamento das imagens relativas reconstruídas. A partir das expectâncias $\mu_{x}$ e $\mu_{y}$, das matrizes de covariância $\Gamma_{x x}$ e $\Gamma_{y y}$ e das matrizes de covariância cruzada $\Gamma_{x y}$ e $\Gamma_{y x}$ obtidas através do método proposto, foram calculadas a matriz $\mathbf{A} \mathbf{e}$ o vetor $\mathbf{b}$ para 3 valores distintos do parâmetro $\kappa$. Na primeira linha são mostrados os resultados das reconstruções corrigidas quando o parâmetro que controla o nível de ruído branco para a inversão da matriz de covariância $\Gamma_{y y}$ foi de $10^{-2}$, enquanto na segunda e terceira linhas estão os resultado para $\kappa=10^{-4}$ e $10^{-8}$, respectivamente.

Para os diversos valores de $\kappa$ foi calculada a diferença entre a imagem relativa es- 


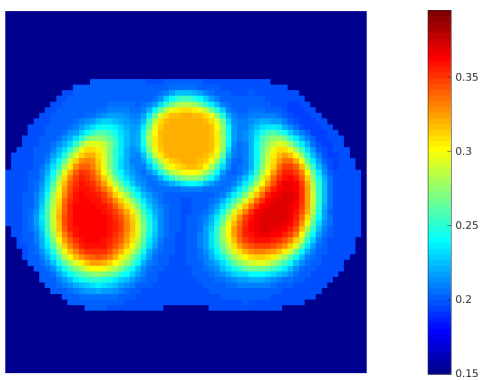

(a) Órgãos intactos, $\kappa=10^{-2}$

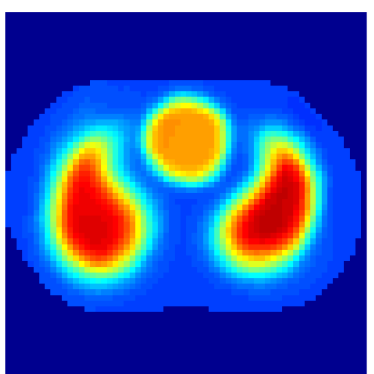

(d) Órgãos intactos, $\kappa=10^{-4}$

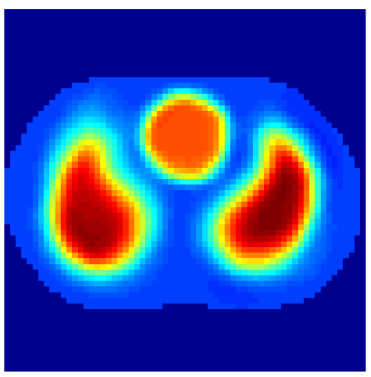

(g) Órgãos intactos, $\kappa=10^{-8}$

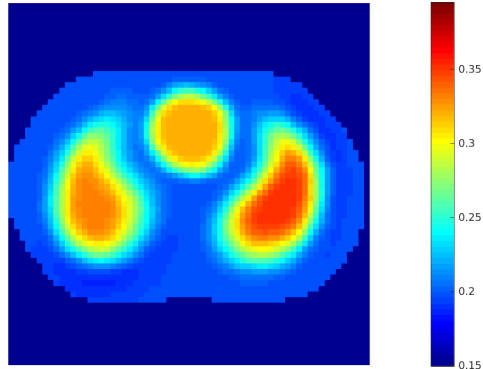

(b) Pulmão direito, corte pequeno, $\kappa=10^{-2}$

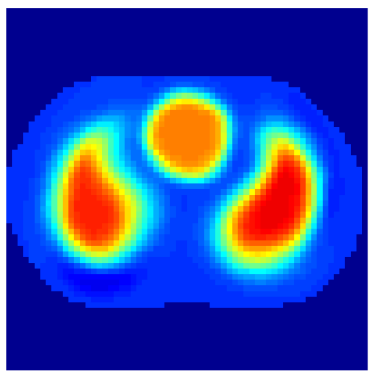

(e) Pulmão direito, corte pequeno, $\kappa=10^{-4}$

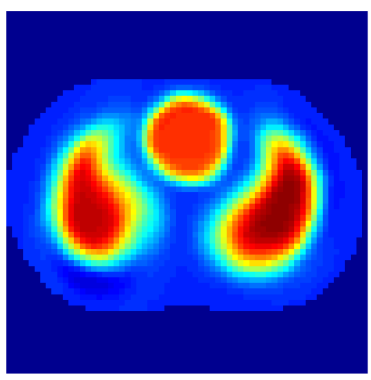

(h) Pulmão direito, corte pequeno, $\kappa=10^{-8}$

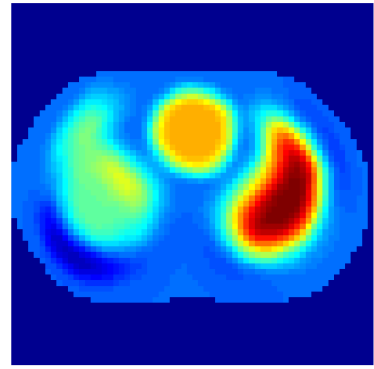

(c) Pulmão direito, grande, $\kappa=10^{-2}$

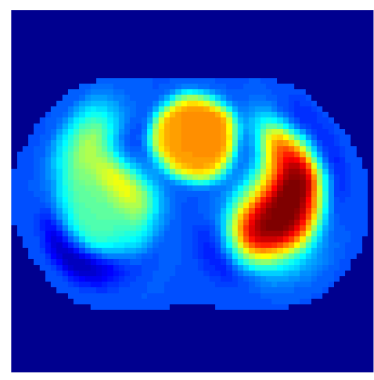

(f) Pulmão direito, grande, $\kappa=10^{-4}$

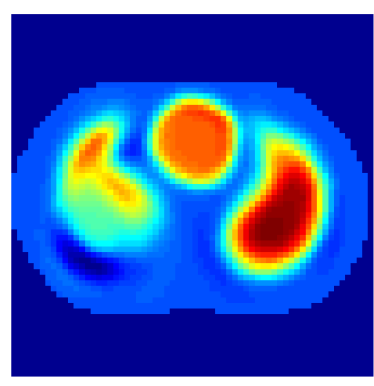

(i) Pulmão direito, corte grande, $\kappa=10^{-8}$

Figura 4.41: Imagens relativas, distribuição de cond. estimada, cuba em formato de tórax humano com coração de ágar e pulmão de melancia com o pós-processamento das imagens.

timada do fantoma onde os órgãos estavam intactos e a imagem relativa estimada do fantoma onde parte do pulmão direito foi retirada. A Figura 4.42 mostra a diferença entre as imagens para o primeiro corte realizado, enquanto a Figura 4.43 mostra a diferença entre as imagens para o corte amplificado.

A Figura 4.40 mostrou o resultado da distribuição de condutividade relativa estimada utilizando o método D-bar. Através destas imagens não é possível observar a posição e formato do coração. Comparando as imagens estimadas também não é possível determinar a localização dos cortes. Diferente das imagens absolutas, as imagens não apresentaram artefatos de condutividade baixa entre os pulmões. 


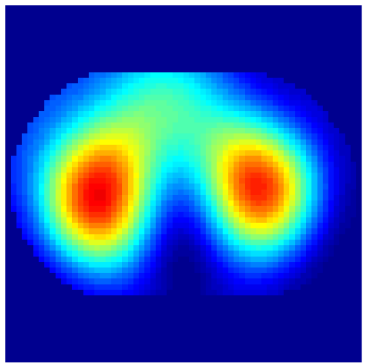

(a) Órgãos intactos, sem ajuste

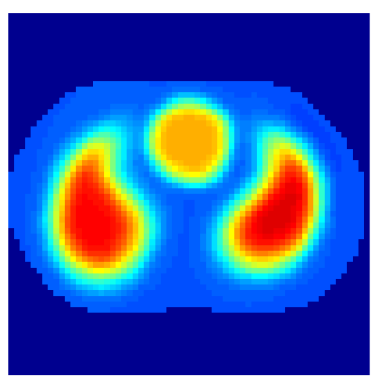

(d) Órgãos intactos, $\kappa=10^{-2}$

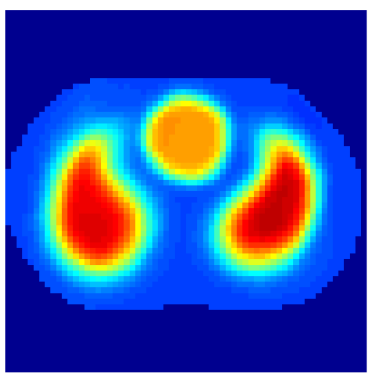

(g) Órgãos intactos, $\kappa=10^{-4}$

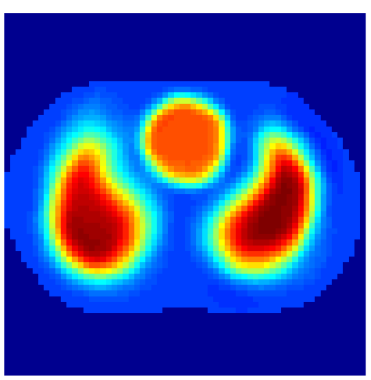

(j) Órgãos intactos, $\kappa=10^{-8}$

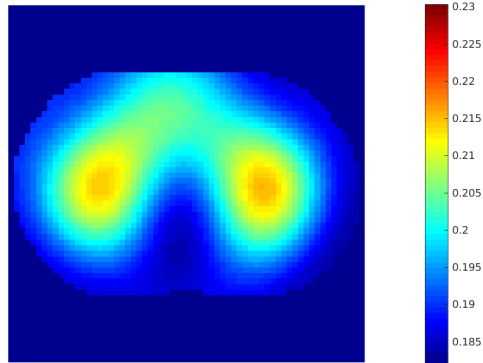

(b) Pulmão direito, corte pequeno, sem ajuste

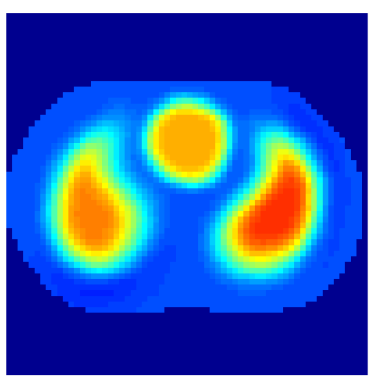

(e) Pulmão direito, corte pequeno, $\kappa=10^{-2}$

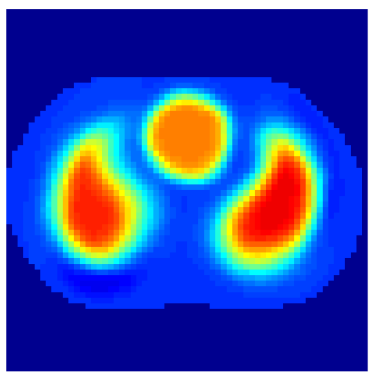

(h) Pulmão direito, corte pequeno, $\kappa=10^{-4}$

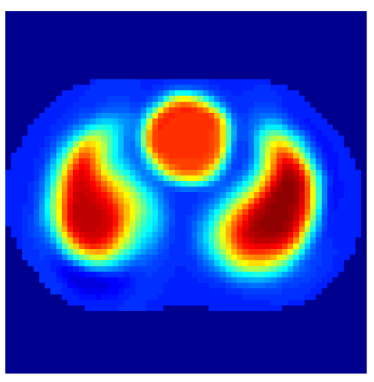

(k) Pulmão direito, corte pequeno, $\kappa=10^{-8}$

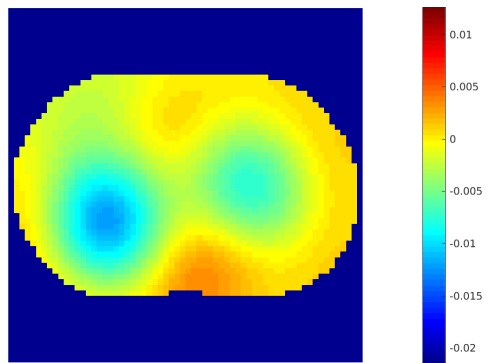

(c) Diferença, corte pequeno, sem ajuste

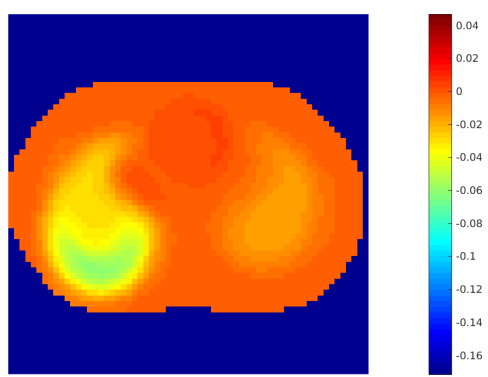

(f) Diferença, corte pequeno, $\kappa=10^{-2}$

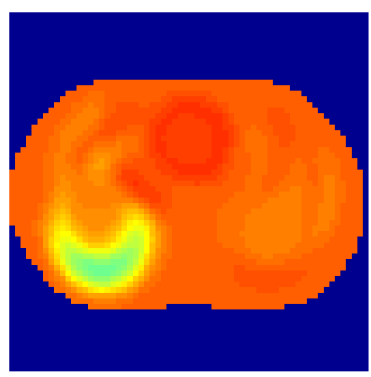

(i) Diferença, corte pequeno, $\kappa=10^{-4}$

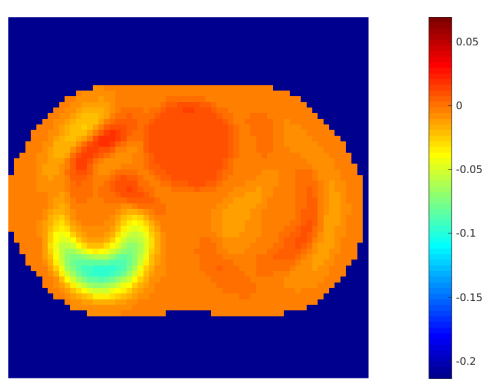

(l) Diferença, corte pequeno, $\kappa=10^{-8}$

Figura 4.42: Diferença entre imagens relativas para corte pequeno no pulmão direito, cuba em formato de tórax humano com coração de ágar e pulmão de melancia com o pós-processamento das imagens.

Com o pós-processamento das imagens relativas através do método proposto, Figura 4.41, a resolução espacial das imagens melhorou consideravelmente. As imagens 


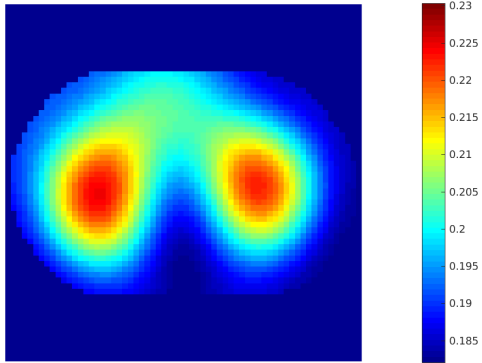

(a) Órgãos intactos, sem ajuste

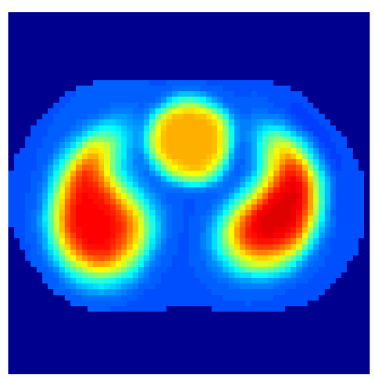

(d) Órgãos intactos, $\kappa=10^{-2}$

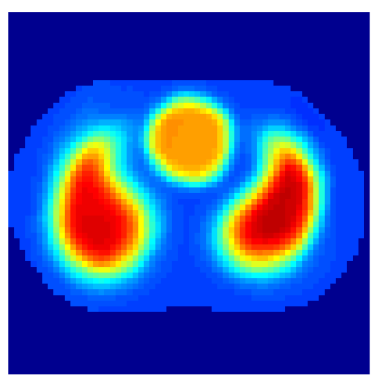

(g) Órgãos intactos, $\kappa=10^{-4}$

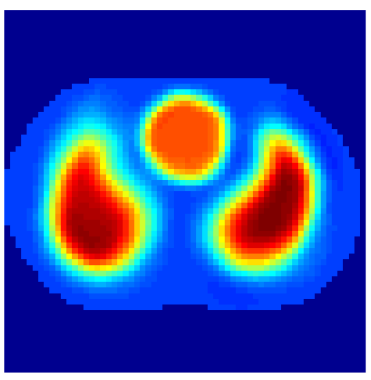

(j) Órgãos intactos, $\kappa=10^{-8}$

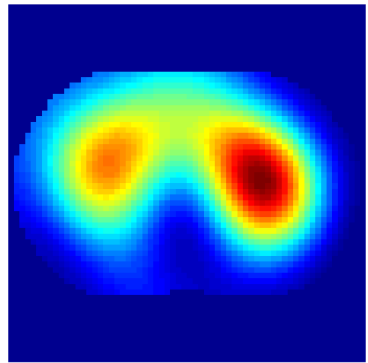

(b) Pulmão direito, grande, sem ajuste

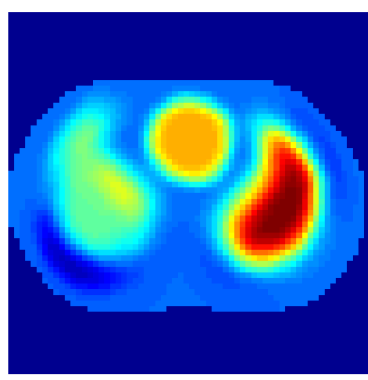

(e) Pulmão direito, grande, $\kappa=10^{-2}$

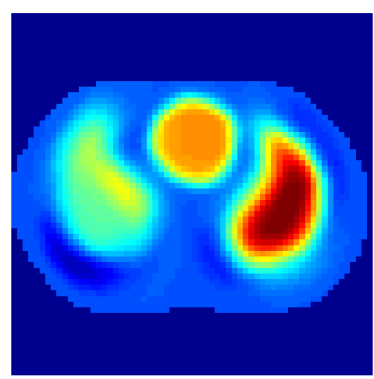

(h) Pulmão direito, grande, $\kappa=10^{-4}$

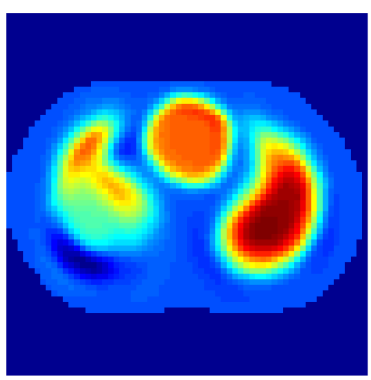

(k) Pulmão direito, grande, $\kappa=10^{-8}$

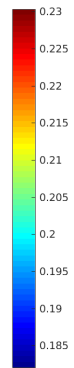

corte

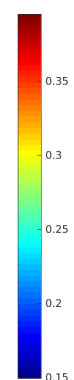

corte

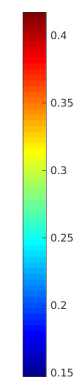

corte

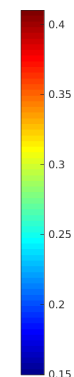

corte

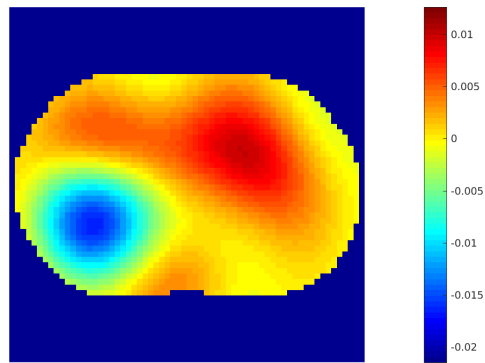

(c) Diferença, corte grande, sem ajuste

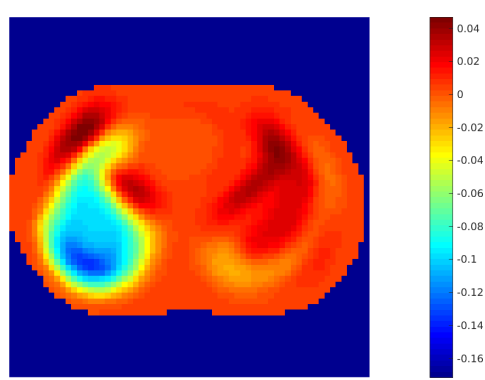

(f) Diferença, corte grande, $\kappa=$ $10^{-2}$

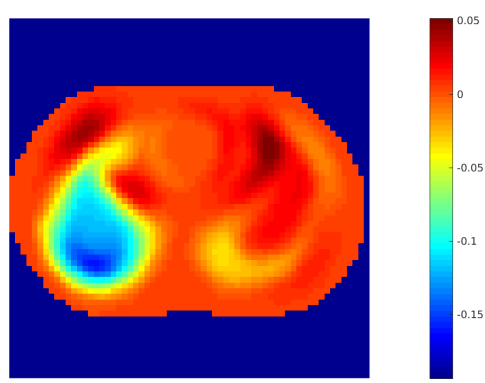

(i) Diferença, corte grande, $\kappa=$ $10^{-4}$

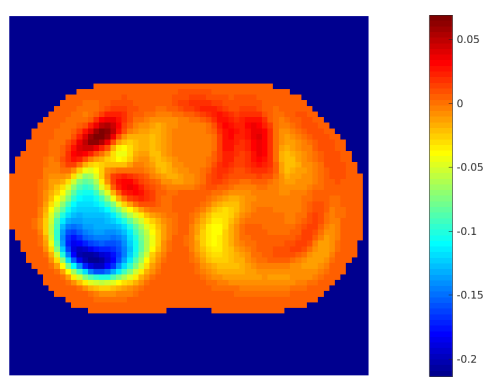

(1) Diferença, corte grande, $\kappa=$ $10^{-8}$

Figura 4.43: Diferença entre imagens relativas para corte grande no pulmão direito, cuba em formato de tórax humano com coração de ágar e pulmão de melancia com o pós-processamento das imagens.

pós-processadas apresentaram formato e posição dos órgãos próximos à posição mostrada no fantoma. Para as imagens relativas foi possível distinguir onde ambos os 
cortes ocorreram. Comparando as imagens para os diversos valores do parâmetro $\kappa$ é possível observar que os cortes no pulmão ficaram mais evidentes nos casos onde o parâmetro utilizado foi menor.

As figuras 4.42 e 4.43 mostram a diferença entre as reconstruções quando o pulmão direito estava intacto e quando cortado. Através destas imagens é possível perceber que tanto o corte pequeno quanto o corte grande foram melhor identificados com o uso do método proposto. Comparando as imagens para os diversos valores do parâmetro $\kappa$ é possível observar que quanto maior o seu valor, mais rígido o método proposto se torna, isto é, mais próximo da distribuição esperada a priori as imagens ficam. 


\section{Capítulo 5}

\section{Conclusões}

Consistente com o objetivo de melhorar a resolução espacial das imagens reconstruídas através do método $D$-bar a partir da introdução de informação a priori, os resultados obtidos mostram que o uso de priors estatísticos, sob a ótica Bayesiana, melhora as imagens reconstruídas pelo método $D$-bar.

A metodologia deste trabalho exigiu o desenvolvimento de modelos refinados dos fantomas experimentais. Para minimizar os efeitos negativos da capacitância parasita da eletrônica do tomógrafo sobre as imagens de TIE, foram incorporados modelos numéricos da capacitância nos eletrodos. Esta inclusão nos modelos de elementos finitos mostrou-se adequada pois permitiu um ajuste das curvas experimentais e de simulação numérica.

O método de introdução de informação a priori na tensão apresentou melhoria marginal das imagens reconstruídas. As melhorias foram observadas na posição e nos contornos dos objetos.

O método de introdução de informação a priori nas imagens reconstruídas apresentou melhora expressiva na resolução espacial das imagens.

Apesar dos métodos propostos terem sido avaliados utilizando o algoritmo $D$-bar, ambos os métodos podem ser aplicados à outros algoritmos de reconstrução.

Em síntese, dois procedimentos de introdução de informação a priori foram testados. Um deles modifica as tensões elétricas e baseia-se no teorema de Bayes. O segundo procedimento modifica a imagem reconstruída e baseia-se numa propriedade do complemento de Schur. O segundo procedimento apresentou melhorias significativas na resolução espacial das imagens. 


\section{Trabalhos futuros}

- Testar o método que utiliza o complemento de Schur nas tensões e na scattering transform;

- Aperfeiçoar o procedimento que utiliza o método do erro de aproximação introduzindo a atualização da matriz de covariância do ruído consistente com o erro de aproximação;

- Utilizar fantomas experimentais mais completos, que apresentem ossos, por exemplo;

- Desenvolver modelos de informação a priori para o tórax humano em condutividade, em potencial elétrico e na scattering transform. 


\section{Apêndice A}

\section{Complemento de Schur}

\section{A.1 Equacionamento do complemento de Schur}

Nesta seção é apresentado a definição dos complementos de Schur e uma das possíveis formas de se calcular a inversa de uma matriz simétrica positiva definida. A demostração é realizada utilizando o método de eliminação de Gauss e segue o equacionamento mostrado em Kaipio e Somersalo [7, Seç. 3.4].

\section{A.1.1 Definição}

Dada a matriz de convariância $\Gamma$,

$$
\Gamma=\left[\begin{array}{cc}
\Gamma_{11} & \Gamma_{12} \\
\Gamma_{21} & \Gamma_{22}
\end{array}\right],
$$

é possível mostrar que a sua matriz inversa pode ser descrita por,

$$
\Gamma^{-1}=\left[\begin{array}{cc}
\tilde{\Gamma}_{22}^{-1} & -\tilde{\Gamma}_{22}^{-1} \Gamma_{12} \Gamma_{22}{ }^{-1} \\
-\tilde{\Gamma}_{22}^{-1} \Gamma_{12} \Gamma_{22}{ }^{-1} & \tilde{\Gamma}_{11}^{-1}
\end{array}\right],
$$

onde

$$
\begin{aligned}
& \tilde{\Gamma}_{22}=\Gamma_{11}-\Gamma_{12} \Gamma_{22}{ }^{-1} \Gamma_{21} \text { e } \\
& \tilde{\Gamma}_{11}=\Gamma_{22}-\Gamma_{21} \Gamma_{11}^{-1} \Gamma_{12} .
\end{aligned}
$$




\section{A.1.2 Lema}

Considerando um sistema linear do tipo,

$$
\Gamma x_{v}=y_{v}
$$

onde $x_{v}$ e $y_{v} \in \mathbb{R}^{n}$ são vetores e $\Gamma \in \mathbb{R}^{n \times n}$ representa uma matriz de covariância, podemos representar este sistema em blocos,

$$
\left[\begin{array}{ll}
\Gamma_{11} & \Gamma_{12} \\
\Gamma_{21} & \Gamma_{22}
\end{array}\right]\left[\begin{array}{l}
x_{1} \\
x_{2}
\end{array}\right]=\left[\begin{array}{l}
y_{1} \\
y_{2}
\end{array}\right]
$$

onde $\Gamma_{11} \in \mathbb{R}^{k \times k}, \Gamma_{22} \in \mathbb{R}^{(n-k) \times(n-k)}, x_{1}$ e $y_{1} \in \mathbb{R}^{k}, x_{2}$ e $y_{2} \in \mathbb{R}^{(n-k)}, k<n$ e $\Gamma_{21}=\Gamma_{12}^{t}$.

Através de uma multiplicação matricial dos blocos apresentados na equação A.2 o seguinte sistema pode ser construído,

$$
\begin{aligned}
& \Gamma_{11} x_{1}+\Gamma_{12} x_{2}=y_{1} \quad \mathrm{e} \\
& \Gamma_{21} x_{1}+\Gamma_{22} x_{2}=y_{2} .
\end{aligned}
$$

Isolando $x_{2}$ na equação A.4, substituindo-o na equação A.3 e utilizando o complemento de Schur $\tilde{\Gamma}_{22}$ temos que,

$$
\begin{aligned}
\Gamma_{11} x_{1}+\Gamma_{12} \boldsymbol{x}_{\mathbf{2}} & =y_{1} \\
\Gamma_{11} x_{1}+\Gamma_{12}\left(\boldsymbol{\Gamma}_{\mathbf{2 2}}{ }^{-\mathbf{1}}\left(\boldsymbol{y}_{\mathbf{2}}-\boldsymbol{\Gamma}_{\mathbf{2 1}} \boldsymbol{x}_{\mathbf{1}}\right)\right) & =y_{1} \\
\Gamma_{11} x_{1} & =y_{1}-\Gamma_{12}\left(\Gamma_{22}{ }^{-1}\left(y_{2}-\Gamma_{21} x_{1}\right)\right) \\
\Gamma_{11} x_{1} & =y_{1}-\Gamma_{12} \Gamma_{22}{ }^{-1} y_{2}+\Gamma_{12} \Gamma_{22}{ }^{-1} \Gamma_{21} x_{1} \\
\Gamma_{11} x_{1}-\Gamma_{12} \Gamma_{22}{ }^{-1} \Gamma_{21} x_{1} & =y_{1}-\Gamma_{12} \Gamma_{22}{ }^{-1} y_{2} \\
\left(\boldsymbol{\Gamma}_{\mathbf{1 1}}-\boldsymbol{\Gamma}_{\mathbf{1 2}} \boldsymbol{\Gamma}_{\mathbf{2 2}}{ }^{-\mathbf{1}} \boldsymbol{\Gamma}_{\mathbf{2 1}}\right) x_{1} & =y_{1}-\Gamma_{12} \Gamma_{22}{ }^{-1} y_{2} \\
\tilde{\boldsymbol{\Gamma}}_{\mathbf{2 2}} x_{1} & =y_{1}-\Gamma_{12} \Gamma_{22}{ }^{-1} y_{2} \\
x_{1} & =\tilde{\Gamma}_{22}^{-1}\left(y_{1}-\Gamma_{12} \Gamma_{22}{ }^{-1} y_{2}\right) \\
x_{1} & =\tilde{\Gamma}_{22}^{-1} y_{1}-\tilde{\Gamma}_{22}^{-1} \Gamma_{12} \Gamma_{22}{ }^{-1} y_{2}
\end{aligned}
$$


Da mesma forma, se isolarmos $x_{1}$ na equação A.3, substituirmos na equação A.4 e utilizando o complemento de Schur $\tilde{\Gamma}_{11}$ temos que,

$$
\begin{aligned}
\Gamma_{21} \boldsymbol{x}_{1}+\Gamma_{22} x_{2} & =y_{2} \\
\Gamma_{21}\left(\boldsymbol{\Gamma}_{\mathbf{1 1}}{ }^{-1}\left(\boldsymbol{y}_{1}-\boldsymbol{\Gamma}_{\mathbf{1 2}} \boldsymbol{x}_{\mathbf{2}}\right)\right)+\Gamma_{22} x_{2} & =y_{2} \\
\Gamma_{22} x_{2} & =y_{2}-\Gamma_{21}\left(\Gamma_{11}^{-1}\left(y_{1}-\Gamma_{12} x_{2}\right)\right) \\
\Gamma_{22} x_{2} & =y_{2}-\Gamma_{21} \Gamma_{11}{ }^{-1} y_{1}+\Gamma_{21} \Gamma_{11}{ }^{-1} \Gamma_{12} x_{2} \\
\Gamma_{22} x_{2}-\Gamma_{21} \Gamma_{11}{ }^{-1} \Gamma_{12} x_{2} & =y_{2}-\Gamma_{21} \Gamma_{11}{ }^{-1} y_{1} \\
\left(\boldsymbol{\Gamma}_{\mathbf{2 2}}-\Gamma_{\mathbf{2 1}} \boldsymbol{\Gamma}_{\mathbf{1 1}}{ }^{-1} \Gamma_{\mathbf{1 2}}\right) x_{2} & =y_{2}-\Gamma_{21} \Gamma_{11}^{-1} y_{1} \\
\tilde{\boldsymbol{\Gamma}}_{11} x_{2} & =y_{2}-\Gamma_{21} \Gamma_{11}^{-1} y_{1} \\
x_{2} & =\tilde{\Gamma}_{11}^{-1}\left(y_{2}-\Gamma_{21} \Gamma_{11}{ }^{-1} y_{1}\right) \\
x_{2} & =\tilde{\Gamma}_{11}^{-1} y_{2}-\tilde{\Gamma}_{11}^{-1} \Gamma_{21} \Gamma_{11}{ }^{-1} y_{1} \\
x_{2} & =-\tilde{\Gamma}_{11}^{-1} \Gamma_{21} \Gamma_{11} y_{1}+\tilde{\Gamma}_{11}^{-1} y_{2}
\end{aligned}
$$

A partir das equações A.5 e A.6 podemos reescrever os sistem mostrado na equação A.2 da seguinte forma,

$$
\begin{aligned}
x_{1} & =\tilde{\Gamma}_{22}^{-1} y_{1}-\tilde{\Gamma}_{22}^{-1} \Gamma_{12} \Gamma_{22}{ }^{-1} y_{2} \\
x_{2} & =-\tilde{\Gamma}_{11}^{-1} \Gamma_{21} \Gamma_{11}{ }^{-1} y_{1}+\tilde{\Gamma}_{11}^{-1} y_{2} \\
{\left[\begin{array}{l}
x_{1} \\
x_{2}
\end{array}\right] } & =\left[\begin{array}{cc}
\tilde{\Gamma}_{22}^{-1} & -\tilde{\Gamma}_{22}^{-1} \Gamma_{12} \Gamma_{22}{ }^{-1} \\
-\tilde{\Gamma}_{11}^{-1} \Gamma_{21} \Gamma_{11}{ }^{-1} & \tilde{\Gamma}_{11}^{-1}
\end{array}\right]\left[\begin{array}{l}
y_{1} \\
y_{2}
\end{array}\right]
\end{aligned}
$$

Então, a inversa da matriz de covariância $\Gamma$ pode ser descrita por,

$$
\Gamma^{-1}=\left[\begin{array}{cc}
\tilde{\Gamma}_{22}^{-1} & -\tilde{\Gamma}_{22}^{-1} \Gamma_{12} \Gamma_{22}{ }^{-1} \\
-\tilde{\Gamma}_{11}^{-1} \Gamma_{21} \Gamma_{11}{ }^{-1} & \tilde{\Gamma}_{11}^{-1}
\end{array}\right] .
$$

E, uma vez que matrizes de covariância são simétricas, podemos observar a seguinte propriedade,

$$
-\tilde{\Gamma}_{11}^{-1} \Gamma_{21} \Gamma_{11}^{-1}=\left(-\tilde{\Gamma}_{22}^{-1} \Gamma_{12} \Gamma_{22}{ }^{-1}\right)^{t} .
$$




\section{A.2 Complemento de Schur e densidade de probabili- dade condicional}

Nesta seção é mostrado como os complementos de Schur podem ser utilizados no cálculo de densidades de probabilidades condicionais. A demostração pode ser encontrada em Kaipio e Somersalo [7, Seç. 3.4] e Anderson [43, Seç. 2.5].

\section{A.2.1 Demonstração}

A densidade de probabilidade conjunta $\pi(x, y)$ de duas variáveis aleatórias gaussianas $X: \Omega \rightarrow \mathbb{R}^{k}$ e $Y: \Omega \rightarrow \mathbb{R}^{n-k}$ pode ser escrita da seguinte forma,

$$
\pi(x, y) \propto \exp \left(-\frac{1}{2}\left[\begin{array}{l}
x-x_{0} \\
y-y_{0}
\end{array}\right]^{t} \Gamma^{-1}\left[\begin{array}{l}
x-x_{0} \\
y-y_{0}
\end{array}\right]\right)
$$

onde $x_{0} \in \mathbb{R}^{k}$ representa a expectância da variável $x, y_{0} \in \mathbb{R}^{n-k}$ a expectância da variável $y$ e $\Gamma \in \mathbb{R}^{n \times n}$ a matriz de covariância do vetor composto pelas variáveis $x$ e $y$.

Dividindo a matriz de covariância $\Gamma$ em blocos temos

$$
\pi(x, y) \propto \exp \left(-\frac{1}{2}\left[\begin{array}{l}
x-x_{0} \\
y-y_{0}
\end{array}\right]^{t}\left[\begin{array}{c}
\Gamma_{11} \Gamma_{12} \\
\Gamma_{22} \Gamma_{22}
\end{array}\right]^{-1}\left[\begin{array}{c}
x-x_{0} \\
y-y_{0}
\end{array}\right]\right)
$$

onde $\Gamma_{11} \in \mathbb{R}^{k \times k}, \Gamma_{22} \in \mathbb{R}^{(n-k) \times(n-k)}, \Gamma_{21}=\Gamma_{12}{ }^{t}$.

Utilizando as simplificações de notação $\tilde{x}=x-x_{0}$ e $\tilde{y}=y-y_{0}$ e substituindo a equação A.9 em A.12 temos,

$$
\begin{aligned}
\pi(x, y) & \propto \exp \left(-\frac{1}{2}\left[\begin{array}{c}
\tilde{x} \\
\tilde{y}
\end{array}\right]^{t}\left[\begin{array}{cc}
\tilde{\Gamma}_{22}^{-1} & -\tilde{\Gamma}_{22}^{-1} \Gamma_{12} \Gamma_{22}{ }^{-1} \\
-\tilde{\Gamma}_{11}^{-1} \Gamma_{21} \Gamma_{11}{ }^{-1} & \tilde{\Gamma}_{11}^{-1}
\end{array}\right]\left[\begin{array}{l}
\tilde{x} \\
\tilde{y}
\end{array}\right]\right) \\
& \propto \exp \left(-\frac{1}{2}\left[\begin{array}{c}
\tilde{x} \\
\tilde{y}
\end{array}\right]^{t}\left[\begin{array}{c}
\tilde{\Gamma}_{22}^{-1} \tilde{x}-\tilde{\Gamma}_{22}^{-1} \Gamma_{12} \Gamma_{22}{ }^{-1} \tilde{y} \\
-\tilde{\Gamma}_{11}^{-1} \Gamma_{21} \Gamma_{11}{ }^{-1} \tilde{x}+\tilde{\Gamma}_{11}^{-1} \tilde{y}
\end{array}\right]\right) \\
& \propto \exp \left(-\frac{1}{2}\left(\tilde{x}^{t} \tilde{\Gamma}_{22}^{-1} \tilde{x}-\tilde{x}^{t}\left(\tilde{\Gamma}_{22}^{-1} \Gamma_{12} \Gamma_{22}{ }^{-1}\right) \tilde{y}-\tilde{y}^{t}\left(\tilde{\Gamma}_{11}^{-1} \Gamma_{21} \Gamma_{11}{ }^{-1}\right) \tilde{x}+\tilde{y}^{t} \tilde{\Gamma}_{11}^{-1} \tilde{y}\right)\right)
\end{aligned}
$$

Uma vez que a matriz de covariância é simétrica a sua inversa também apresenta a 
mesma propriedade, então temos que,

$$
\tilde{x}^{t}\left(\tilde{\Gamma}_{22}^{-1} \Gamma_{12} \Gamma_{22}{ }^{-1}\right) \tilde{y}=\tilde{y}^{t}\left(\tilde{\Gamma}_{11}^{-1} \Gamma_{21} \Gamma_{11}{ }^{-1}\right) \tilde{x}
$$

Por tanto, a densidade de probabilidade conjunta de duas variáveis aleatórias gaussianas pode ser expressa por,

$$
\pi(x, y) \propto \exp \left(-\frac{1}{2}\left(\tilde{x}^{t} \tilde{\Gamma}_{22}^{-1} \tilde{x}-2 \tilde{x}^{t}\left(\tilde{\Gamma}_{22}^{-1} \Gamma_{12} \Gamma_{22}^{-1}\right) \tilde{y}+\tilde{y}^{t} \tilde{\Gamma}_{11}^{-1} \tilde{y}\right)\right)
$$

Em seu livro Kaipio e Somersalo [7, Teorema 3.5] mostra que reordenando a equação A.17 é possível descrever a densidade de probabilidade conjunta $\pi(x, y)$ por,

$$
\begin{aligned}
\pi(x, y) & \propto \exp \left(-\frac{1}{2}\left(\left(\tilde{x}-\Gamma_{12} \Gamma_{22}^{-1} \tilde{y}\right)^{t} \tilde{\Gamma}_{22}^{-1}\left(\tilde{x}-\Gamma_{12} \Gamma_{22}{ }^{-1} \tilde{y}\right)+c\right)\right), \\
c & =\tilde{y}^{t}\left(\tilde{\Gamma}_{11}^{-1}-\Gamma_{22}^{-1} \Gamma_{21} \tilde{\Gamma}_{22}^{-1} \Gamma_{12} \Gamma_{22}^{-1}\right) \tilde{y} .
\end{aligned}
$$

Utilizando o teorema de Bayes e conhecido o evento $y$ temos que a densidade de probabilidade condicional $\pi(x \mid y)$ é proporcional a densidade de probabilidade conjunta,

$$
\pi(x \mid y) \propto \pi(x, y) \propto \exp \left(-\frac{1}{2}\left(\left(\tilde{x}-\Gamma_{12} \Gamma_{22}{ }^{-1} \tilde{y}\right)^{t} \tilde{\Gamma}_{22}^{-1}\left(\tilde{x}-\Gamma_{12} \Gamma_{22}^{-1} \tilde{y}\right)+c\right)\right)
$$

Então temos que,

$$
\begin{aligned}
\pi(x \mid y) & \propto \exp \left(-\frac{1}{2}\left(\left(\tilde{x}-\Gamma_{12} \Gamma_{22}{ }^{-1} \tilde{y}\right)^{t} \tilde{\Gamma}_{22}^{-1}\left(\tilde{x}-\Gamma_{12} \Gamma_{22}{ }^{-1} \tilde{y}\right)+c\right)\right) \\
& \propto \exp \left(-\frac{1}{2}\left(\tilde{x}-\Gamma_{12} \Gamma_{22}{ }^{-1} \tilde{y}\right)^{t} \tilde{\Gamma}_{22}^{-1}\left(\tilde{x}-\Gamma_{12} \Gamma_{22}{ }^{-1} \tilde{y}\right)\right) \exp \left(-\frac{1}{2} c\right)
\end{aligned}
$$

Como o termo $c$ depende apenas de $y, y_{0}$ e $\Gamma$, todos constantes, a parte exponencial $\exp \left(-\frac{1}{2} c\right)$ pode ser considerado constante e excluída da equação de proporcionalidade, restando assim

$$
\pi(x \mid y) \propto \exp \left(-\frac{1}{2}\left(\tilde{x}-\Gamma_{12} \Gamma_{22}{ }^{-1} \tilde{y}\right)^{t} \tilde{\Gamma}_{22}^{-1}\left(\tilde{x}-\Gamma_{12} \Gamma_{22}{ }^{-1} \tilde{y}\right)\right)
$$


Substituindo a simplificação de notação aplicada $\tilde{x}=\left(x-x_{0}\right)$ e $\tilde{y}=\left(y-y_{0}\right)$ temos,

$$
\pi(x \mid y) \propto \exp \left(-\frac{1}{2}\left(\left(x-x_{0}\right)-\Gamma_{12} \Gamma_{22}{ }^{-1}\left(y-y_{0}\right)\right)^{t} \tilde{\Gamma}_{22}^{-1}\left(\left(x-x_{0}\right)-\Gamma_{12} \Gamma_{22}{ }^{-1}\left(y-y_{0}\right)\right)\right)
$$

Assim, reordenando a equação A.22, temos,

$$
\begin{array}{r}
\pi(x \mid y) \propto \exp \left(-\frac{1}{2}(x-\bar{x})^{t} \tilde{\Gamma}_{22}^{-1}(x-\bar{x})\right) \\
\bar{x}=x_{0}+\Gamma_{12} \Gamma_{22}^{-1}\left(y-y_{0}\right)
\end{array}
$$

Por fim, se representarmos os blocos $\Gamma_{11}, \Gamma_{12}, \Gamma_{21}$ e $\Gamma_{22}$ da matriz de covariância $\Gamma$ utilizando as matrizes de covariância e de covariância cruzada de $x$ e $y$,

$$
\Gamma=\left[\begin{array}{ll}
\Gamma_{11} & \Gamma_{12} \\
\Gamma_{21} & \Gamma_{22}
\end{array}\right]=\left[\begin{array}{c}
\Gamma_{x x} \Gamma_{x y} \\
\Gamma_{y x} \Gamma_{y y}
\end{array}\right]
$$

onde $\Gamma_{x x} \in \mathbb{R}^{n \times n}, \Gamma_{y y} \in \mathbb{R}^{(n-k) \times(n-k)}, \Gamma_{21}=\Gamma_{12}{ }^{t}$, podemos reescrever a equação A.23 da seguinte forma,

$$
\begin{aligned}
\pi(x \mid y) & \propto \exp \left(-\frac{1}{2}(x-\bar{x})^{t} \tilde{\Gamma}_{y y}^{-1}(x-\bar{x})\right), \\
\tilde{\Gamma}_{y y} & =\Gamma_{x x}-\Gamma_{x y} \Gamma_{y y}{ }^{-1} \Gamma_{y x} \mathrm{e} \\
\bar{x} & =x_{0}+\Gamma_{x y} \Gamma_{y y}{ }^{-1}\left(y-y_{0}\right),
\end{aligned}
$$

e concluir que a distribuição condicional pode ser representada pela distribuição normal,

$$
\pi(x \mid y)=N\left(\bar{x}, \tilde{\Gamma}_{y y}\right)
$$

O valor esperado $\bar{x}$ e a matriz de covariância $\tilde{\Gamma}_{y y}$ também são descritos na literatura por $\mu_{x \mid y}$ (média condicional) e $\Gamma_{x \mid y}$ (covariância condicional). Para mais detalhes, a dedução também pode ser encontrada em Ouellette [42, Seç. 6.1] e Anderson [43, Teorema 2.5.1]. 


\section{A.3 Estimativa do máximo a posteriori}

A partir de um conjunto de dados $Y=y$ e conhecido o modelo estatístico que descreve as variáveis aleatórias Gaussianas $X$ e $Y$ e suas correlações, uma estimativa do máximo a posteriori (MAP) de $x$ que leva em consideração $y$ pode ser calculada por,

$$
X_{\text {map }}=\underset{x}{\operatorname{argmax}}(\pi(x \mid y)) .
$$

Substituindo a densidade de probabilidade $\pi(x \mid y)$ pela equação A.25 mostrada na seção A.2 temos que,

$$
X_{\text {map }}=\underset{x}{\operatorname{argmax}}\left(\exp \left(-\frac{1}{2}(x-\bar{x})^{t} \tilde{\Gamma}_{y y}^{-1}(x-\bar{x})\right)\right) .
$$

Aplicando o logaritmo natural e eliminando as constantes multiplicativas, a função de maximização descrita na equação A.28 pode ser reescrita por meio de uma função de minimização equivalente,

$$
\begin{aligned}
X_{\text {map }} & =\underset{x}{\operatorname{argmax}}(\pi(x \mid y)) \\
& =\underset{x}{\operatorname{argmin}}(-\ln (\pi(x \mid y)) \\
& =\underset{x}{\arg \min _{x}}\left(-\ln \left(\exp \left(-\frac{1}{2}(x-\bar{x})^{t} \tilde{\Gamma}_{y y}^{-1}(x-\bar{x})\right)\right)\right) \\
& =\underset{x}{\operatorname{argmin}}\left((x-\bar{x})^{t} \tilde{\Gamma}_{y y}^{-1}(x-\bar{x})\right) .
\end{aligned}
$$

Como mostrado na seção A.2 a matriz $\tilde{\Gamma}_{y y}$ é uma matriz de covariância, sendo assim uma matriz simétrica positiva semidefinida. Para o cálculo da sua matriz inversa $\tilde{\Gamma}_{y y}^{-1}$ é necessário que um ruído branco seja adicionado à sua diagonal principal, tornando-a não singular e inversível,

$$
X_{\text {map }}=\underset{x}{\operatorname{argmin}}\left((x-\bar{x})^{t}\left(\tilde{\Gamma}_{y y}+\eta I\right)^{-1}(x-\bar{x})\right),
$$

onde $\eta$ controla o nível de ruído branco e $I$ é a matriz identidade.

Considerando a adição do ruído branco a matriz inversa $\tilde{\Gamma}_{y y}^{-1}$ torna-se simétrica 
positiva semidefinida e a seguinte propriedade pode ser utilizada,

$$
a^{t} A a \geq 0, \quad \text { para } \quad \forall a \neq 0,
$$

onde $a \in \mathbb{R}^{n_{a}}$ é um vetor qualquer, $A \in \mathbb{R}^{n_{a} \times n_{a}}$ uma matriz positiva semidefinida e $n_{a}$ um número inteiro positivo.

Utilizando a propriedade mostrada na equação A.30 temos que $X_{\text {map }}$ (eq. A.29) será sempre maior ou igual a zero e consequentemente a distribuição de $x$ que maximiza a estimativa de máximo a posteriori pode ser descrita pela média da distribuição condicional $\pi(x \mid y)$,

$$
\begin{aligned}
X_{\text {map }} & =\underset{x}{\operatorname{argmax}}(\pi(x \mid y)) \\
& =\underset{x}{\arg \min _{x}}\left((x-\bar{x})^{t} \tilde{\Gamma}_{y y}^{-1}(x-\bar{x})\right) \\
& =\underset{x}{\operatorname{argmin}}\left((x-\bar{x})^{t}\left(\tilde{\Gamma}_{y y}+\kappa I\right)^{-1}(x-\bar{x})\right) \\
& =\bar{x} \\
& =x_{0}+\Gamma_{x y} \Gamma_{y y}{ }^{-1}\left(y-y_{0}\right) .
\end{aligned}
$$




\section{Bibliografia}

[1] Richard C. Aster, Brian Borchers, and Clifford H. Thurber. Parameter estimation and inverse problems. Elsevier, 2005.

[2] Albert Tarantola. Inverse problem theory and methods for model parameter estimation. Society for Industrial and Applied Mathematics, 2005. ISBN $9780898715729,0898715725$.

[3] Margaret Cheney, David Isaacson, and Jonathan C. Newell. Electrical impedance tomography. SIAM review, 41(1):85-101, 1999.

[4] Paivi J. Vauhkonen. Image reconstruction in three-dimensional electrical impedance tomography. Tese de doutorado, University of Kuopio, 2004.

[5] M. Dodd and J. L. Mueller. A real-time D-bar algorithm for 2-D electrical impedance tomography data. Inverse Probl. Imag., 8(4):1013-1031, 2014.

[6] J. Mueller and S. Siltanen. Linear and Nonlinear Inverse Problems with Practical Applications. Society for Industrial and Applied Mathematics, Philadelphia, PA, 2012.

[7] Jari Kaipio and Erkki Somersalo. Statistical and computational inverse problems, volume 160. Springer, 2005.

[8] Erick Darío León Bueno Camargo. Desenvolvimento de algoritmo de imagens absolutas de Tomografia por Impedância Elétrica para uso clínico. Tese de doutorado, Escola Politécnica da Universidade de São Paulo, São Paulo, 2013.

[9] David S. Holder. Electrical impedance tomography: methods, history and applications. CRC Press, 2004.

[10] B. H. Brown. Electrical impedance tomography (eit): a review. Journal of medical engineering \& technology, 2003. 
[11] Thiago de Castro Martins, Andre Kubagawa Sato, Fernando Silva de Moura, Erick Dario León Bueno de Camargo, Olavo Luppi Silva, Talles Batista Rattis Santos, Zhanqi Zhao, Knut Moeller, Marcelo Brito Passos Amato, Jennifer L. Mueller, Raul Gonzalez Lima, and Marcos de Sales Guerra Tsuzuki. A review of electrical impedance tomography in lung applications: Theory and algorithms for absolute images. Annual Reviews in Control, 2019.

[12] Kimmo Karhunen, Aku Seppänen, Anssi Lehikoinen, Paulo J. M. Monteiro, and Jari P. Kaipio. Electrical resistance tomography imaging of concrete. Cement and Concrete Research, 40(1):137 - 145, 2010. ISSN 0008-8846. doi: https:/ /doi.org/10. 1016/j.cemconres.2009.08.023.

[13] L. M. Heikkinen, J. Kourunen, T. Savolainen, P. J. Vauhkonen, J. P. Kaipio, and M. Vauhkonen. Real time three-dimensional electrical impedance tomography applied in multiphase flow imaging. Measurement Science and Technology, 17(8): 2083-2087, jul 2006. doi: 10.1088/0957-0233/17/8/005.

[14] M. M. Mellenthin, J. L. Mueller, E. D. L. B. de Camargo, F. S. de Moura, T. B. R. Santos, R. G. Lima, S. J. Hamilton, P. A. Muller, and M. Alsaker. The ace1 electrical impedance tomography system for thoracic imaging. IEEE Transactions on Instrumentation and Measurement, pages 1-14, 2018. ISSN 0018-9456. doi: 10.1109/TIM.2018.2874127.

[15] Michelle M. Mellenthin. The active complex electrode (ACE1) electrical impedance tomography system and anatomically inspired modeling of electrode skin contact impedance. Tese de doutorado, School of Biomedical Engineering of Colorado State University, Fort Collins, EUA, 2016.

[16] Jaakko Malmivuo and Robert Plonsey. Bioelectromagnetism: principles and applications of bioelectric and biomagnetic fields. Oxford University Press, 1995.

[17] Fernando Silva de Moura. Estimação não linear de estado através do Unscented Kalman Filter na tomografia por impedância elétrica. Tese de doutorado, Escola Politécnica da Universidade de São Paulo, São Paulo, 2012.

[18] J. Rosell, J. Colominas, P. Riu, R. Pallas-Areny, and J. G. Webster. Skin impedance 
from $1 \mathrm{hz}$ to $1 \mathrm{mhz}$. IEEE Transactions on Biomedical Engineering, 35(8):649-651, Aug 1988.

[19] Kuo-Sheng Cheng, D. Isaacson, J. C. Newell, and D. G. Gisser. Electrode models for electric current computed tomography. IEEE Transactions on Biomedical Engineering, 36(9):918-924, Sept 1989.

[20] Ping Hua, Eung Je Woo, John G. Webster, and Willis J. Tompkins. Finite element modeling of electrode-skin contact impedance in electrical impedance tomography. Biomedical Engineering, IEEE Transactions on, 40(4):335-343, 1993.

[21] Päivi J. Vauhkonen, Marko Vauhkonen, Tuomo Savolainen, and Jari P. Kaipio. Three-dimensional electrical impedance tomography based on the complete electrode model. IEEE Transactions on Biomedical Engineering, 46(9):1150-1160, 1999.

[22] Olavo Luppi Silva. Muscle contraction detection using electrical impedance tomography. Tese de doutorado, Escola Politécnica da Universidade de São Paulo, São Paulo, 2012.

[23] A. Björck. Numerical methods for least squares problems. Society for Industrial and Applied Mathematics, 1996.

[24] S. Siltanen, J. L. Mueller, and D. Isaacson. An implementation of the reconstruction algorithm of $\mathrm{A}$ Nachman for the $2 \mathrm{D}$ inverse conductivity problem. Inverse Probl., 16(3):681-699, 2000.

[25] J. L. Mueller, P. A. Muller, M. M. Mellenthin, E. DeBoer, R. Murthy, M. Capps, M. Alsaker, R. Deterding, and S. Sagel. A method of estimating regions of air trapping from electrical impedance tomography data. Physiological Measurement, 39(5):05NT01, 2018.

[26] P. A. Muller, J. L. Mueller, M. M. Mellenthin, R. Murthy, M. Capps, B.D. Wagner, M. Alsaker, R. Deterding, S. Sagel, and J. Hoppe. Evaluation of surrogate measures of pulmonary function derived from electrical impedance tomography data in children with cystic fibrosis. Physiological Measurement, 39(4):045008, 2018. 
[27] E. K. Murphy and J. L. Mueller. Effect of domain shape modeling and measurement errors on the 2-d d-bar method for eit. IEEE Transactions on Medical Imaging, 28(10):1576-1584, Oct 2009. ISSN 0278-0062. doi: 10.1109/TMI.2009.2021611.

[28] D. Isaacson, J. L. Mueller, J. C. Newell, and S. Siltanen. Reconstructions of chest phantoms by the D-bar method for electrical impedance tomography. IEEE T. Med. Imaging, 23(7):821-828, 2004.

[29] Kim Knudsen, Matti Lassas, Jennifer Mueller, and Samuli Siltanen. D-bar method for electrical impedance tomography with discontinuous conductivities. SIAM Journal of Applied Mathematics, 67:893-913, 01 2007. doi: 10.1137/060656930.

[30] N. J. Avis and D. C. Barber. Incorporating a priori information into the Sheffield filtered backprojection algorithm. Physiol. Meas., 16(3A):A111-A122, 1995.

[31] U. Baysal and B. M. Eyüboglu. Use of a priori information in estimating tissue resistivities - a simulation study. Phys. Med. and Biol., 43(12):3589-3606, 1998.

[32] H. Dehghani, D. C. Barber, and I. Basarab-Horwath. Incorporating a priori anatomical information into image reconstruction in electrical impedance tomography. Physiol. Meas., 20(1):87-102, 1999.

[33] D. Ferrario, B. Grychtol, A. Adler, J. Sola, S. H. Bohm, and M. Bodenstein. Toward morphological thoracic EIT: Major signal sources correspond to respective organ locations in CT. IEEE T. Med. Imaging, 59(11):3000-3008, 2012.

[34] D. Flores-Tapia and S. Pistorius. Electrical impedance tomography reconstruction using a monotonicity approach based on a priori knowledge. In Engineering in Medicine and Biology Society (EMBC), 2010 Annual International Conference of the IEEE, pages 4996-4999, Aug 2010.

[35] J. P. Kaipio, V. Kolehmainen, M. Vauhkonen, and E. Somersalo. Inverse problems with structural prior information. Inverse Probl., 15(3):713, 1999.

[36] F. S. Moura, J. C. C. Aya, A. T. Fleury, M. B. P. Amato, and R. G. Lima. Dynamic imaging in electrical impedance tomography of the human chest with online transition matrix identification. IEEE Transactions on Biomedical Engineering, 57(2): 422-431, Feb 2010. ISSN 0018-9294. doi: 10.1109/TBME.2009.2032529. 
[37] M. Vauhkonen, D. Vadasz, P. A. Karjalainen, E. Somersalo, and J. P. Kaipio. Tikhonov regularization and prior information in electrical impedance tomography. IEEE T. Med. Imaging, 17(2):285-293, 1998.

[38] A. Nissinen, L. M. Heikkinen, and J. P. Kaipio. The bayesian approximation error approach for electrical impedance tomography-experimental results. Measurement Science and Technology, 19(1), 2008.

[39] T. Sousa, F. Moura, I. Hoffmann, E. D. L. B. Camargo, R. Lima, A. Martins, D. Fantoni, and C. Biasi. In vivo admitivity measurements for regularizations in electrical impedance tomography. In Proceedings of COBEM 2011, 21st International Congress of Mechanical Engineering, pages 24-28, 2011.

[40] M. Alsaker and J. L. Mueller. A D-bar algorithm with a priori information for 2-dimensional electrical impedance tomography. SIAM J. Imaging Sci, 9(4):16191654, 2016.

[41] Fuzhen Zhang. The Schur complement and its applications, volume 4. Springer Science \& Business Media, 2006.

[42] Diane Valerie Ouellette. Schur complements and statistics. Linear Algebra and its Applications, 36:187-295, 1981.

[43] T. W. Anderson. An Introduction to Multivariate Statistical Analysis. Wiley Series in Probability and Statistics. Wiley, 2003. ISBN 9780471360919. 